Decaimento dos autovalores de operadores integrais gerados por núcleos positivos definidos

José Claudinei Ferreira 



\title{
Decaimento dos autovalores de operadores integrais gerados por núcleos positivos definidos ${ }^{1}$
}

\author{
José Claudinei Ferreira
}

Orientador: Prof. Dr. Valdir Antonio Menegatto

Dissertação apresentada ao Instituto de Ciências Matemáticas e de Computação - ICMC-USP, como parte dos requisitos para obtenção do título de Mestre em Matemática.

"VERSÃO REVISADA APÓS DEFESA "

Data da Defesa:

$11 / 02 / 2008$

Visto do Orientador:

\section{USP - São Carlos \\ Fevereiro/2008}

\footnotetext{
${ }^{1} \mathrm{O}$ autor teve apoio financeiro da FAPESP.
} 

Aos meus pais, Vicente e Jovina. 



\section{Agradecimentos}

Agradeço primeiramente a Deus pela minha existência. Em seguida aos meus pais, Vicente Ferreira Sobrinho e Jovina Gomes Ferreira, pela dedicação, pela educação e pelo esforço para comigo e meus irmãos. Agradeço-os ainda pelo exemplo de pessoas íntegras que são. Aos meus irmãos, José Carlos Ferreira, Márcio José Ferreira, Geildo Gomes Ferreira e Jonas Gomes Ferreira, e às minhas irmãs, Maria Roseli Ferreira, Maria Josiane Ferreira Gomes e Eliane Ferreira Gomes, sou grato por muitos motivos. No meio acadêmico agradeço à minha grande professora e amiga Izabel Parolin pelo incentivo e auxilio ao meu ingresso no curso de graduação, o qual divulgou a possibilidade de ingresso na universidade aos meus amigos e familiares. No meio acadêmico agradeço em especial ao meu orientador de iniciação científica Prof. Dr. José Roberto Nogueira e ao Prof. Dr. Messias Meneguette Junior. Ao meu orientador de mestrado Prof. Dr. Valdir Antonio Menegatto expresso a minha sincera gratidão pela orientação e pela dedicação. Agradeço ainda a todos os amigos e colegas, em especial, ao Daniel, Fausto, Kenji, Mateus e ao Tiago. Por fim agradeço à FAPESP pelo apoio financeiro. 

Inicialmente, estudamos alguns resultados clássicos da teoria dos núcleos positivos definidos e alguns resultados pertinentes. Estudamos em seguida, o Teorema de Mercer e algumas de suas generalizações e conseqüências, incluindo a caracterização da transformada de Fourier de um núcleo positivo definido com domínio $\mathbb{R}^{m} \times \mathbb{R}^{m}, m \geq 1$. O trabalho traz um enfoque especial nos núcleos cujo domínio é um subconjunto não-compacto de $\mathbb{R}^{m} \times \mathbb{R}^{m}$, uma vez que os demais casos são considerados de maneira extensiva na literatura. Aplicamos esses estudos na análise do decaimento dos autovalores de operadores integrais gerados por núcleos positivos definidos. 

Firstly, we study some classical results from the theory of positive definite kernels along with some related results. Secondly, we focus on generalizations of Mercer's theorem and some of their implications. Special attention is given to the cases where the domain of the kernel is not compact, once the other cases are considered consistently in the literature. We include a characterization for the Fourier transform of a positive definite kernel on $\mathbb{R}^{m} \times \mathbb{R}^{m}, m \geq 1$. Finally, we apply the previous study in the analysis of decay rates for eigenvalues of integral operators generated by positive definite kernels. 

Funções e núcleos positivos definidos são muito utilizados em vários ramos da Matemática, tais como Análise de Fourier, Teoria dos Operadores, Teoria de Probabilidades, Problemas de Valores de Contorno para Equações Diferenciais Parciais, Teoria da Aproximação, entre outros, cada contexto exigindo uma formulação adequada para o conceito. Este é antigo e provavelmente tem suas origens na Teoria dos Operadores Integrais. A referência [2] contém material introdutório sobre os núcleos positivos definidos enquanto que o artigo [39], apesar de antigo, apresenta uma revisão histórica do assunto, descrevendo vários contextos onde eles são empregados. Sua utilização em Teoria da Aproximação pode ser ratificada em [8, 36, 37].

Neste trabalho analisamos propriedades espectrais de operadores integrais definidos por núcleos positivos definidos em $X \times X$, onde $X$ é um subconjunto Lebesguemensurável de $\mathbb{R}^{m}, m \geq 1$. No caso em que $X$ é compacto, muitas propriedades destes operadores podem ser encontradas na literatura. Um exemplo disto é o já antigo Teorema de Mercer e suas generalizações ([20, 26, 28]). Os resultados mais recentes de que temos notícia são aqueles descritos nas referências $[3,4,5,6,7,9]$, entre outras, onde o contexto inclui apenas o caso $m=1$ mas permite que o subconjunto $X$ seja não compacto. Nestes artigos, três questões fundamentais são consideradas:

(i) se $X$ é não-compacto, estabelecer condições sobre o núcleo gerador do operador integral de modo que a conclusão do Teorema de Mercer original ainda vale em algum sentido.

(ii) no caso em que $X=\mathbb{R}$, assumindo-se que o Teorema de Mercer é válido, obter informações sobre a transformada de Fourier do núcleo gerador.

(iii) assumindo-se alguma condição de suavidade sobre o núcleo gerador do operador, analisar o decaimento dos autovalores do operador integral.

Neste trabalho pretendemos obter generalizações dos resultados acima para o caso em que $X$ é um subconjunto de $\mathbb{R}^{m}, m \geq 1$, incluindo o caso em que $X$ é não-compacto. 
O trabalho própriamente dito divide-se nas seguintes partes:

No Capítulo 1 apresentamos os resultados técnicos utilizados no trabalho; os resultados clássicos de Análise Funcional são descritos já adaptados ao contexto.

No Capítulo 2, inicialmente apresentamos duas formulações para o conceito de positividade definida, analisando possíveis relações entre elas. Em um segundo estágio, demonstramos uma versão do Teorema de Mercer, já adaptada aos domínios que consideramos aqui, analisando algumas relações entre núcleos positivos definidos e sua transformada de Fourier.

No Capítulo 3 analisamos o decaimento dos autovalores de operadores integrais associados a núcleos positivos definidos, estendendo resultados da literatura para o caso multidimensional. 


\section{Sumário}

Introdução

xiii

1 Preliminares 1

1.1 Matrizes não-negativas definidas . . . . . . . . . . . . . . . . . . . . 1

1.2 Alguns resultados de topologia e análise . . . . . . . . . . . . . . 2

1.3 Alguns resultados da teoria da medida . . . . . . . . . . . . . . 7

1.4 Espaços de Banach e de Hilbert . . . . . . . . . . . . . . . . . . . . . . 10

1.5 Um pouco de teoria espectral . . . . . . . . . . . . . . . . . . . 14

1.6 Operadores do tipo Hilbert-Schmidt . . . . . . . . . . . . . . . 15

1.7 Transformada de Fourier . . . . . . . . . . . . . . . . . . . . . . 19

2 Núcleos Positivos Definidos $\quad 21$

2.1 Núcleos positivos definidos . . . . . . . . . . . . . . . . . . 21

2.2 Núcleos $L^{2}$-positivos definidos . . . . . . . . . . . . . . . . . 25

2.3 Representação de núcleos $L^{2}$-positivos definidos . . . . . . . . . . . . . 29

2.4 Núcleos $L^{2}$-positivos definidos: generalizações . . . . . . . . . . . . . . 35

2.5 Núcleos $L^{2}$-positivos definidos: caracterização . . . . . . . . . . . . . . 40

2.6 Núcleos $L^{2}$-positivos definidos e a Transformada de Fourier . . . . . . . 47

3 Decaimento de autovalores $\quad 57$

3.1 Introdução . . . . . . . . . . . . . . . . . . . . . . . . 57

3.2 Aproximação por operadores de posto finito . . . . . . . . . . . . 58

3.3 A raiz quadrada de um operador . . . . . . . . . . . . . . . 59

3.4 Operadores de posto finito . . . . . . . . . . . . . . . . 62

3.5 Núcleos lipschitzianos . . . . . . . . . . . . . . . . . 66

3.6 Núcleos diferenciáveis . . . . . . . . . . . . . . . . . . . . . . . . 70

3.7 Análise das estimativas . . . . . . . . . . . . . . . . . . . . . . . . . . . 79 
3.8 Considerações finais . . . . . . . . . . . . . . . . . . . . . . 82

Referências Bibliográficas $\quad 83$

$\begin{array}{lr}\text { Índice Remissivo } & 87\end{array}$ 


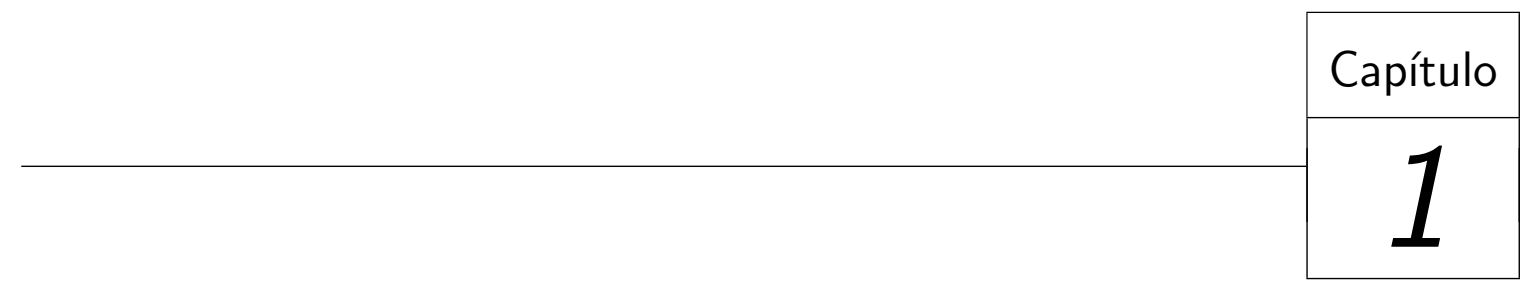

Preliminares

Este capítulo contém resultados básicos, clássicos e técnicos utilizados no trabalho. Enquanto alguns são enunciados em sua formulação mais geral, outros mais técnicos já aparecem descritos na maneira como serão empregados.

O critério adotado para a inclusão ou não de demonstração dos resultados foi baseado no número de fontes encontradas para os mesmos. Havendo mais de uma referência onde o resultado aparece, optamos pela não inclusão de demonstração do mesmo.

\subsection{Matrizes não-negativas definidas}

Uma das formulações de positividade definida recai no conceito de positividade definida para matrizes. Tal conceito é estudado detalhadamente em [17], uma referência sólida para a teoria de matrizes e suas aplicações. O símbolo $A_{n \times n}$ denota uma matriz genérica de ordem $n$ com entradas em $\mathbb{C}$ enquanto que $\bar{x}, x \in \mathbb{C}^{n}$, corresponde ao vetor obtido de $x$, conjugando-se cada uma de suas coordenadas.

Definição 1.1.1. Uma matriz $A_{n \times n}$ é não-negativa definida quando

$$
\bar{x} A x^{T} \geq 0, \quad x \in \mathbb{C}^{n} .
$$

Propriedades 1.1.2. Seja $A_{n \times n}$ uma matriz não-negativa definida. Valem as seguintes afirmações:

(i) Qualquer submatriz principal de $A$ é não-negativa definida;

(ii) Todo autovalor de $A$ é não-negativo; 
(iii) As entradas na diagonal principal de $A$ são todas não-negativas; em símbolos, $A_{i i} \geq 0, i=1,2, \ldots, n$;

(iv) $\mathrm{O}$ traço de $A$, denotado por $\operatorname{tr}(A)$, é não-negativo. Ainda, se $S \subset\{1,2, \ldots, n\}$ é não-vazio e $A(S)$ é a matriz obtida de $A$, excluindo-se as linhas e as colunas de $A$ correspondentes aos índices de $\{1,2, \ldots, n\} \backslash S$, então $\operatorname{tr}(A(S))$ é não-negativo;

$(v)$ Se $S \subset\{1,2, \ldots, n\}$ é não-vazio, então o determinante de $A(S)$ é não-negativo; (vi) $A$ é hermiteana, ou seja, $A_{i j}=\overline{A_{j i}}, i, j=1,2, \ldots, n$.

\subsection{Alguns resultados de topologia e análise}

Começamos com os resultados clássicos de Weierstrass ([27, p.167]) e Dini ([23, p.211]).

Teorema 1.2.1 (Weierstrass). Se $X$ é um espaço topológico compacto e $f: X \rightarrow \mathbb{R}$ é contínua, então existem $x_{0}, x_{1} \in X$ tais que $f\left(x_{0}\right) \leq f(x) \leq f\left(x_{1}\right), x \in X$.

Teorema 1.2.2 (Dini). Sejam $X$ um espaço topológico compacto e $\left\{f_{n}\right\}$ uma seqüência de funções reais contínuas definidas em $X$. Se $\left\{f_{n}\right\}$ é monótona e pontualmente convergente para uma função contínua $f: X \rightarrow \mathbb{R}$, então a convergência é uniforme.

Demonstração: Suponhamos, sem perda de generalidade, que a seqüência é decrescente. Cada função $f_{n}-f$ é contínua e a seqüência $\left\{f_{n}-f\right\}$ é decrescente. Como $X$ é compacto, o teorema anterior justifica a existência de $x_{n} \in X$ tal que

$$
M_{n}:=f_{n}\left(x_{n}\right)-f\left(x_{n}\right)=\max _{x \in X}\left\{f_{n}(x)-f(x)\right\} .
$$

Claramente, $\left\{M_{n}\right\}$ é uma seqüência decrescente de termos não-negativos. Logo, converge para algum $c \geq 0$. Mostremos que $c=0$. Da compacidade de $X$ novamente, passando para uma subseqüência, se necessário, podemos assumir que $\left\{x_{n}\right\}$ converge para algum $x_{0} \in X$. Como

$$
M_{k}=f_{k}\left(x_{k}\right)-f\left(x_{k}\right) \leq f_{m}\left(x_{k}\right)-f\left(x_{k}\right), \quad k \geq m,
$$

fazendo $k \rightarrow \infty$ e usando a continuidade das funções envolvidas, deduzimos que $c \leq$ $f_{m}\left(x_{0}\right)-f\left(x_{0}\right)$. Fazendo agora $m \rightarrow \infty$, obtemos $c \leq 0$. Finalmente, fixado $\epsilon>0$, existe $N \in \mathbb{N}$ tal que $M_{n}<\epsilon$, quando $n \geq N$. Portanto,

$$
0 \leq f_{n}(x)-f(x) \leq f_{n}\left(x_{n}\right)-f\left(x_{n}\right)=M_{n}<\epsilon, \quad x \in X
$$

Segue que

$$
\left|f_{n}(x)-f(x)\right|<\epsilon, \quad x \in X, \quad n \geq N,
$$


ou seja, $\left\{f_{n}\right\}$ converge uniformemente para $f$.

No que segue $|\cdot|$ denota a norma euclidiana de $\mathbb{R}^{m}$. O resultado abaixo é uma versão do teorema anterior no caso em que $X$ não é compacto.

Teorema 1.2.3. Sejam $X$ um subconjunto fechado e ilimitado de $\mathbb{R}^{m}$ e $\left\{f_{n}\right\}$ uma seqüência monótona de funções contínuas definidas em $X$, pontualmente convergente para uma função contínua $f$. Se

$$
\lim _{|x| \rightarrow \infty} f_{n}(x)=\lim _{|x| \rightarrow \infty} f(x)=0, \quad n=1,2, \ldots,
$$

então $\left\{f_{n}\right\}$ converge uniformemente para $f$.

Demonstração: Sem perda de generalidade vamos assumir que a seqüência $\left\{f_{n}\right\}$ é decrescente. Cada função $f_{n}-f$ é contínua e não-negativa. Se $\lim _{|x| \rightarrow \infty}\left(f_{n}(x)-f(x)\right)=0$ existe $x_{n} \in X$ tal que

$$
M_{n}:=\max _{x \in X}\left\{f_{n}(x)-f(x)\right\}=f_{n}\left(x_{n}\right)-f\left(x_{n}\right) .
$$

Claramente, $\left\{M_{n}\right\}$ é uma seqüência decrescente de termos não-negativos, pois

$$
f_{n}(x)-f(x)>f_{n+1}(x)-f(x), \quad x \in X, \quad n=1,2 \ldots .
$$

Logo, ela converge para algum $c \geq 0$. Vamos mostrar que $c=0$, considerando dois casos:

Caso 1: $\left\{x_{n}\right\}$ é ilimitada. Neste caso, podemos assumir que $\left|x_{n}\right| \rightarrow \infty$, quando $n \rightarrow \infty$, e conseqüentemente, a desigualdade acima implica que

$$
\lim _{n \rightarrow \infty}\left(f_{n}\left(x_{n}\right)-f\left(x_{n}\right)\right)=0 .
$$

Logo, $c=0$.

Caso 2: $\left\{x_{n}\right\}$ é limitada. Neste caso, existe um cubo fechado $Q$, tal que $\left\{x_{n}\right\} \subset Q \cap X$. Como o cubo é compacto, podemos assumir que $\left\{x_{n}\right\}$ converge para $x_{0} \in Q \cap X$. Daí, procedendo-se como no teorema anterior, segue que $c=0$.

Em qualquer caso, dado $\epsilon>0$, existe $N \in \mathbb{N}$ tal que

$$
0 \leq f_{n}(x)-f(x) \leq f_{n}\left(x_{n}\right)-f\left(x_{n}\right)=M_{n}<\epsilon, \quad n \geq N, \quad x \in X,
$$

ou seja, $\left\{f_{n}\right\}$ converge uniformemente para $f$.

Uma demonstração mais formal da teorema anterior é como segue. Defina um novo conjunto $Y$ dado pela expressão

$$
Y=X \cup\{\infty\}
$$


e considere agora as funções $f_{n}, n=1,2, \ldots$, e $f$ como funções de $Y$ em $\mathbb{R}$, onde $f_{n}(\infty):=0$ e $f(\infty):=0$. Neste caso podemos considerar o conjunto $Y$ como sendo uma compactificação de $X$ ([27, p.183]). Como as funções $f_{n}, n=1,2, \ldots$, e $f$ são funções contínuas em $Y$, o Teorema de Dini nos dá o resultado.

Uma prova para o teorema a seguir pode ser encontrada em ([27, p.130]).

Teorema 1.2.4. Sejam X um espaço topológico e $M$ um espaço métrico. Se uma seqüência de funções de $X$ em $M$ converge uniformemente para uma função $f: X \rightarrow M$ e cada elemento da seqüência é uma função contínua, então $f$ é contínua.

O restante dos resultados referem-se à ordem de convergência de algumas seqüências e séries especiais. Eles são utilizados no estudo do decaimento dos autovalores de operadores integrais no Capítulo 3.

Lema 1.2.5. Se $m, q, \gamma$ e $p$ são números reais e $p>1$, então existe $N_{0} \in \mathbb{N}$ tal que

$$
\left(p(n+1)^{m}+q\right)^{\gamma} \leq p^{\gamma+1} n^{m \gamma}, \quad n \geq N_{0} .
$$

Demonstração: Basta notar que

$$
\lim _{n \rightarrow \infty} \frac{\left(p(n+1)^{m}+q\right)^{\gamma}}{p^{\gamma+1} n^{m \gamma}}=\frac{1}{p}
$$

e usar que $p^{-1}<1$.

Uma seqüência $\left\{a_{n}\right\}$ de números reais é eventualmente não-crescente quando existe $n_{0}$ tal que $\left\{a_{n_{0}+n}\right\}$ é não-crescente e de termos não-negativos.

Lema 1.2.6. Seja $\left\{a_{n}\right\}$ uma seqüência eventualmente não-crescente. Assuma que existem constantes $m, N_{0}, p, q \in \mathbb{N}, p \geq 1, \gamma \in \mathbb{R}$ e $C \geq 0$, tais que

$$
\sum_{j=k(n)+q+1}^{\infty} n^{\gamma} a_{j} \leq C, \quad n \geq N_{0},
$$

onde $k(n) \in\left\{0,1, \ldots, p n^{m}\right\}$. Então o conjunto $\left\{n^{m+\gamma} a_{2 p n^{m}+q}: n=1,2, \ldots\right\}$ é limitado. Em particular, existe $N_{1}$ tal que

$$
0 \leq n^{m+\gamma} a_{2 p n^{m}+q} \leq C, \quad n \geq N_{1}
$$

Demonstração: Seja $n_{0}$ um inteiro positivo com a seguinte característica: a subseqüência de termos não negativos $\left\{a_{n_{0}+j}\right\}$ é não-crescente. Defina $N_{1}=\max \left\{n_{0}, N_{0}\right\}$ e note que

$$
\sum_{j=p n^{m}+q+1}^{\infty} n^{\gamma} a_{j} \leq \sum_{j=k(n)+q+1}^{\infty} n^{\gamma} a_{j} \leq C, \quad n \geq N_{1} .
$$


Utilizando as hipóteses e observando que o vetor $\left(n^{\gamma} a_{p n^{m}+q+1}, n^{\gamma} a_{p n^{m}+q+2}, \ldots, n^{\gamma} a_{2 p n^{m}+q}\right)$ possui $p n^{m} \geq n^{m}$ coordenadas, temos que

$$
\begin{aligned}
n^{m+\gamma} a_{2 p n^{m}+q} & =n^{m} n^{\gamma} a_{2 p n^{m}+q} \\
& \leq n^{\gamma} a_{p n^{m}+q+1}+\cdots+n^{\gamma} a_{2 p n^{m}+q} \\
& \leq \sum_{j=p n^{m}+q+1}^{\infty} n^{\gamma} a_{j} \leq C, \quad n \geq N_{1} .
\end{aligned}
$$

Logo, $\left\{n^{m+\gamma} a_{2 p n^{m}+q}\right\}_{n=1}^{\infty} \subset[-r, r]$, onde $r=\max \left\{C,\left|n^{m+\gamma} a_{2 p n^{m}+q}\right|: n=1,2, \ldots, N_{1}\right\}$, o que conclui a prova do lema.

Teorema 1.2.7. Seja $\left\{a_{n}\right\}$ uma seqüência eventualmente não-crescente. Assuma que existem constantes $m, N_{0}, p, q \in \mathbb{N}, p \geq 1, \gamma \in \mathbb{R}$ e $C \geq 0$, tais que

$$
\sum_{j=k(n)+q+1}^{\infty} n^{\gamma} a_{j} \leq C, \quad n \geq N_{0},
$$

onde $k(n) \in\left\{0,1, \ldots, p n^{m}\right\}$. Então o conjunto $\left\{n^{1+\gamma / m} a_{n}: n=1,2 \ldots\right\}$ é limitado. Em particular, existe um inteiro $N$ tal que

$$
0 \leq n^{1+\gamma / m} a_{n} \leq(2 p)^{1+\gamma / m} C, \quad n \geq N
$$

Demonstração: Do Lema 1.2.6 já temos que o conjunto $\left\{n^{m+\gamma} a_{2 p n^{m}+q}, n=1,2, \ldots\right\}$ é limitado. Na verdade, a prova do lema revela que existe $N_{1}>0$ tal que

$$
0 \leq n^{m+\gamma} a_{2 p n^{m}+q} \leq C, \quad n \geq N_{1}
$$

Para cada natural $s>q$, existe $n_{s} \in \mathbb{N}$ tal que

$$
2 p n_{s}^{m}+q \leq s \leq 2 p\left(n_{s}+1\right)^{m}+q
$$

Com isso temos que existe um natural $N_{2} \geq N_{1}$ tal que

$$
s^{1+\gamma / m} a_{s} \leq\left(2 p\left(n_{s}+1\right)^{m}+q\right)^{1+\gamma / m} a_{2 p n_{s}^{m}+q}, \quad s \geq N_{2} .
$$

Do Lema 1.2.5, com $p_{1}=2 p$ e $\gamma_{1}=1+\gamma / m$, segue que existe $N_{3} \geq N_{1}+N_{2}$ tal que

$$
s^{\gamma_{1}} a_{s} \leq p_{1}^{\gamma_{1}+1} n_{s}^{m \gamma_{1}} a_{2 p n_{s}^{m}+q}=p_{1}^{\gamma_{1}+1} n_{s}^{m+\gamma} a_{2 p n_{s}^{m}+q} \leq p_{1}^{\gamma_{1}+1} C, \quad s \geq N_{3} .
$$

Desta forma, o conjunto $\left\{n^{1+\gamma / m} a_{n}, n=N_{3}, N_{3}+1, \ldots\right\}$ é limitado.

Na definição abaixo recordamos dois conceitos usuais. 
Definição 1.2.8. Sejam $\left\{a_{n}\right\}$ e $\left\{b_{n}\right\}$ duas seqüências numéricas. Assuma que $b_{n}=0$ apenas para um número finito de indices. Dizemos que $a_{n}=o\left(b_{n}\right)$ quando a seqüência $\left\{a_{n} b_{n}^{-1}\right\}$ converge para 0 . Dizemos que $a_{n}=O\left(b_{n}\right)$ quando $\left\{a_{n} b_{n}^{-1}, n=N_{0}, N_{0}+1, \ldots\right\}$ é limitada, para algum $N_{0} \in \mathbb{N}$.

Podemos restabelecer parte do Teorema 1.2.7 utilizando a linguagem da definição acima.

Corolário 1.2.9. Se a seqüência $\left\{a_{n}\right\}$ satisfaz às hipóteses do Teorema 1.2.7, então $a_{n}=O\left(n^{-1-\gamma / m}\right)$.

Outra conseqüência do mesmo teorema é como segue.

Corolário 1.2.10. Se $\left\{a_{n}\right\}$ é eventualmente não-crescente e a série $\sum_{n=1}^{\infty} a_{n}$ é convergente, então $a_{n}=o\left(n^{-1}\right)$.

Demonstração: Sem perda de generalidade podemos assumir que $\left\{a_{n}\right\}$ é não-crescente de termos não-negativos. A convergência da série implica que $\lim _{n \rightarrow \infty} \sum_{j=n}^{\infty} a_{j}=0$. Desta forma, dado $\epsilon>0$, existe $N=N(\epsilon)$ tal que

$$
\sum_{j=n+1}^{\infty} a_{j} \leq \epsilon, \quad n \geq N .
$$

Aplicando o Teorema 1.2.7, $\operatorname{com} \gamma=0, p=1$ e $q=0$, segue que existe $N_{1}=N_{1}(\epsilon) \geq N$ tal que

$$
0 \leq n a_{n} \leq 2 \epsilon, \quad n \geq N_{1}
$$

Isto completa a prova.

Corolário 1.2.11. Seja $\left\{a_{n}\right\}$ uma seqüência eventualmente não-crescente. Se para cada $C \geq 0$ existem constantes $m, N=N(C), p, q \in \mathbb{N}$, com $p \geq 1$, e $\gamma \in \mathbb{R}$, tais que

$$
\sum_{j=k(n)+q+1}^{\infty} n^{\gamma} a_{j} \leq C, \quad n \geq N
$$

onde $k(n) \in\left\{0,1, \ldots, p n^{m}\right\}$, então $a_{n}=o\left(n^{-1-\gamma / m}\right)$.

Demonstração: Seja $\left\{C_{j}\right\}$ uma seqüência de números reais positivos convergindo para 0. Do Teorema 1.2.7 segue que, para cada $C_{j}$, existe $N_{j}$ tal que

$$
0 \leq n^{1+\gamma / m} a_{n} \leq(2 p)^{1+\gamma / m} C_{j}, \quad n \geq N_{j}
$$

e o corolário segue. 


\subsection{Alguns resultados da teoria da medida}

Nesta seção, revisamos alguns conceitos e resultados básicos da teoria da medida. Inicialmente, introduzimos os espaços $L^{p}$ usuais ([12, p.181]).

Definição 1.3.1. Se $(X, \mathcal{M}, \mu)$ é um espaço de medida e $p \in[1, \infty)$, definimos

$$
L^{p}(X):=\left\{f: X \rightarrow \mathbb{C}: f \text { é } \mu \text {-mensurável e }\|f\|_{L^{p}(X)}<\infty\right\}
$$

onde

$$
\|f\|_{L^{p}(X)}:=\left[\int_{X}|f(x)|^{p} d \mu(x)\right]^{1 / p} .
$$

O conjunto $L^{p}(X)$ torna-se um espaço vetorial quando identificamos quaisquer duas funções $f$ e $g$ de $L^{p}(X)$ que são idênticas a menos de um conjunto em $\mathcal{M}$ de medida nula (o termo equivalente para tal identificação é $f$ e g são iguais quase sempre ou, simplificadamente, $f=g$ q.s.).

No caso em que $X=\mathbb{R}^{m}$, a menos de especificação em contrário, $\mathcal{M}$ é a família de todos os conjuntos mensuráveis de $X$ e $\mu$ é a medida de Lebesgue usual de $\mathbb{R}^{m}$. Observação análoga vale no caso em que $X$ é subconjunto de $\mathbb{R}^{m}$. No contexto de $L^{p}(X \times X)$, a medida $\mu$ é a medida produto correspondente.

As afirmações incluídas no teorema abaixo estão provadas em [12, p.183, 245].

Teorema 1.3.2. Valem as seguintes propriedades:

(i) O espaço $\left(L^{p}(X),\|\cdot\|_{L^{p}(X)}\right)$ é um espaço de Banach;

(ii) O conjunto das funções de classe $C^{\infty}$ e de suporte compacto em $\mathbb{R}^{m}$, é um subconjunto denso do espaço $L^{p}\left(\mathbb{R}^{m}\right)$. Em particular, $L^{p}\left(\mathbb{R}^{m}\right) \cap L^{q}\left(\mathbb{R}^{m}\right)$ é um subconjunto denso de ambos, $L^{p}\left(\mathbb{R}^{m}\right)$ e $L^{q}\left(\mathbb{R}^{m}\right)$.

A estrutura sobre a qual a maioria dos resultados deste trabalho são desenvolvidos é aquela onde a norma é oriunda de um produto interno. Neste sentido, o exemplo abaixo registra a estrutura ideal.

Exemplo 1.3.3. Se $(X, \mathcal{M}, \mu)$ é espaço de medida, então $L^{2}(X)$ é um espaço de Hilbert com produto interno dado por

$$
\langle f, g\rangle_{L^{2}(X)}:=\int_{X} f(x) \overline{g(x)} d \mu(x), \quad f, g \in L^{2}(X) .
$$

Por simplicidade vamos denotar o produto interno de $L^{2}(X)$ por $\langle\cdot, \cdot\rangle$.

No desenvolvimento do trabalho fazemos muitas manipulações de integrais, por isso terminamos esta seção com alguns resultados úteis sobre o assunto ([12]). 
Teorema 1.3.4 (Desigualdade de Hölder). Seja $(X, \mathcal{M}, \mu)$ um espaço de medida. Seja $p \in[1, \infty)$ e considere o expoente conjugado $q$ de $p$. Se $f$ e $g$ são funções mensuráveis em $X$, então

$$
\|f g\|_{L^{1}(X)} \leq\|f\|_{L^{p}(X)}\|g\|_{L^{q}(X)} .
$$

Em particular, se $f \in L^{p}(X)$ e $g \in L^{q}(X)$, então $f g \in L^{1}(X)$.

Corolário 1.3.5. Sejam $(X, \mathcal{M}, \mu), p$ e q como no teorema anterior. Se $\mu(X)<\infty e$ $p<q<\infty$, então $L^{q}(X) \subset L^{p}(X)$ e $\|f\|_{L^{p}(X)} \leq\|f\|_{L^{q}(X)} \mu(X)^{(q-p) / p q}$.

No que segue, $L^{+}(X)$ indica o conjunto das funções mensuráveis em $X$ que são não-negativas (podem até assumir o valor $\infty$ ).

Teorema 1.3.6 (Convergência Monótona). Se $\left\{f_{n}\right\}$ é uma seqüência não-decrescente de $L^{+}(X)$, então

$$
\int_{X} \lim _{n \rightarrow \infty} f_{n}=\lim _{n \rightarrow \infty} \int_{X} f_{n}
$$

Teorema 1.3.7. Se $f \in L^{+}(X)$, então $\int_{X} f=0$ se, e somente se, $f=0$ q.s..

Teorema 1.3.8. Se $f_{n} \rightarrow f$ em $L^{1}(X)$, então existe uma subseqüência $\left\{f_{n_{j}}\right\}$ tal que $f_{n_{j}} \rightarrow f$ q.s..

Teorema 1.3.9 (Convergência Dominada). Seja $\left\{f_{n}\right\}$ uma seqüência em $L^{1}(X)$ que satisfaz:

(i) $\lim _{n \rightarrow \infty} f_{n}=f$ q.s.;

(ii) Existe uma função $g \in L^{1}(X)$ tal que tal que $\left|f_{n}\right| \leq g$ q.s., para todo $n$.

Então $f \in L^{1}(X) e$

$$
\int_{X} f=\lim _{n \rightarrow \infty} \int_{X} f_{n}
$$

Os seguintes resultados que garantem a iteração de integrais em espaços produtos podem ser encontrados em [12, p.67].

Teorema 1.3.10 (Fubini-Tonelli). Sejam $(X, \mathcal{M}, \mu)$ e $(Y, \mathcal{N}, \nu)$ espaços de medida $\sigma$-finitos.

(i) (Tonelli) Se $f \in L^{+}(X \times Y)$, então

$$
g(x)=\int_{Y} f(x, y) d \nu(y) \in L^{+}(X), \quad h(y)=\int_{X} f(x, y) d \mu(x) \in L^{+}(Y),
$$

e vale a fórmula

$$
\int_{X \times Y} f d(\mu \times \nu)=\int_{X}\left[\int_{Y} f(x, y) d \nu(y)\right] d \mu(x)=\int_{Y}\left[\int_{X} f(x, y) d \mu(x)\right] d \nu(y) .
$$


(ii) (Fubini) Se $f \in L^{1}(X \times Y)$, então $f(x, \cdot) \in L^{1}(Y)$ para quase todo $x, f(\cdot, y) \in$ $L^{1}(X)$ para quase todo $y$, e as funções definidas quase sempre

$$
g(x)=\int_{Y} f(x, y) d \nu(y), \quad h(y)=\int_{X} f(x, y) d \mu(x),
$$

são elementos de $L^{1}(X)$ e $L^{1}(Y)$ respectivamente. Além disso, a fórmula do item $(i)$ vale.

Teorema 1.3.11 (Mudança de Variáveis em Integrais). Sejam $\Omega$ um subconjunto aberto de $\mathbb{R}^{m}$ e $G: \Omega \rightarrow \mathbb{R}^{m}$ um $C^{1}$-difeomorfismo. Se $f$ é uma função Lebesguemensurável em $G(\Omega)$, então $f \circ G$ é Lebesgue-mensurável em $\Omega$. Se $f \geq 0$ ou $f \in$ $L^{1}(G(\Omega))$, então

$$
\int_{G(\Omega)} f(x) d x=\int_{\Omega} f \circ G(x)|\operatorname{det}(J G(x))| d x,
$$

onde $\operatorname{det}(J G(x))$ é o determinante da matriz do jacobiano de $G(x)$.

Teorema 1.3.12. Sejam c e $C$ constantes positivas, $B:=\left\{x \in \mathbb{R}^{m}:|x|<c\right\}$ e $f$ uma função Lebesgue-mensurável em $\mathbb{R}^{m}$.

(i) Se $|f(x)| \leq C|x|^{-\alpha}, x \in B$, para algum $\alpha<m$, então $f \in L^{1}(B)$. Entretanto, se $|f(x)| \geq C|x|^{-m}, x \in B$, então $f \notin L^{1}(B)$;

(ii) Se $|f(x)| \leq C|x|^{-\alpha}, x \notin B$, para algum $\alpha>m$, então $f \in L^{1}\left(\mathbb{R}^{m} \backslash B\right)$. Entretanto, se $|f(x)| \geq C|x|^{-m}, x \notin B$, então $f \notin L^{1}\left(\mathbb{R}^{m} \backslash B\right)$.

Utilizamos o Teorema 1.3.13 abaixo na Seção 2.2 na análise do conceito de positividade definida. Se $x_{0} \in \mathbb{R}^{m}$ e $r>0, C\left[x_{0}, r\right]$ denota o cubo fechado de centro $x_{0}$ e lados de comprimento $r>0$ paralelos aos eixos coordenados.

Teorema 1.3.13 (Diferenciação de Lebesgue). Sejam $X$ um subconjunto de $\mathbb{R}^{m}$ e $f: X \rightarrow \mathbb{C}$ uma função. Assuma que $C\left[x_{0}, r\right] \subset X$. Se $f$ é contínua em $C\left[x_{0}, s\right]$, $s \in(0, r)$, então

$$
\lim _{s \rightarrow 0^{+}} \frac{1}{\left|C\left[x_{0}, s\right]\right|} \int_{C\left[x_{0}, s\right]} f(y) d y=f\left(x_{0}\right),
$$

onde $\left|C\left[x_{0}, s\right]\right|$ é o volume de $C\left[x_{0}, s\right]$.

Não faremos a demonstração deste teorema aqui, já que o mesmo é conhecido e pode ser encontrado em uma forma mais geral em [12, p.98] ou em [42, p.100].

Teorema 1.3.14. Seja $f: \mathbb{R}^{m} \rightarrow \mathbb{C}$ Lebesgue-mensurável. Assuma que $f \in L^{1}\left(\mathbb{R}^{m}\right)$ ou $f \in L^{+}\left(\mathbb{R}^{m}\right)$. Se $f$ é radial, ou seja, existe $g:(0, \infty) \rightarrow[0, \infty)$ tal que $f(x)=g(|x|)$, $x \in \mathbb{R}^{m}$, então

$$
\int_{\mathbb{R}^{m}} f(x) d x=\frac{2 \pi^{m / 2}}{\Gamma(m / 2)} \int_{0}^{\infty} g(r) r^{m-1} d r
$$




\subsection{Espaços de Banach e de Hilbert}

Esta seção contém os pré-requisitos de Análise Funcional utilizados ao longo do trabalho. Somente alguns poucos resultados não tão populares são premiados com alguma demonstração.

Observamos que em todo o trabalho utilizamos apenas espaços de Hilbert separáveis de dimensão infinita, com excessão de $\mathbb{R}^{m}$. Logo, alguns dos resultados aqui cotados podem aparecer em formulações mais gerais na literatura.

Começamos com a desigualdade de Cauchy-Schwarz e uma de suas conseqüências envolvendo convergência. Em concordância com as notações anteriores, vamos escrever $\|\cdot\|_{\mathcal{X}}$ para denotar a norma do espaço vetorial normado $\mathcal{X}$.

Teorema 1.4.1. Se $\mathcal{H}$ é um espaço de Hilbert com produto interno $\langle\cdot, \cdot\rangle_{\mathcal{H}}$, então

$$
\left|\langle x, y\rangle_{\mathcal{H}}\right| \leq\|x\|_{\mathcal{H}}\|y\|_{\mathcal{H}}, \quad x, y \in \mathcal{H}
$$

Teorema 1.4.2. Seja $\mathcal{H}$ um espaço de Hilbert. Se $\left\{x_{n}\right\}$ e $\left\{y_{n}\right\}$ são seqüências de $\mathcal{H}$ que convergem para $x$ e $y$, respectivamente, então $\left\{\left\langle x_{n}, y_{n}\right\rangle_{\mathcal{H}}\right\}$ converge para $\langle x, y\rangle_{\mathcal{H}}$.

Usaremos a seguinte versão da desigualdade de Bessel ([43, p.34]).

Teorema 1.4.3. Se $\mathcal{H}$ é um espaço de Hilbert e $\left\{x_{n}\right\}$ é um subconjunto ortonormal de $\mathcal{H}$, então

$$
\|y\|_{\mathcal{H}}^{2} \geq \sum_{n=1}^{\infty}\left|\left\langle y, x_{n}\right\rangle_{\mathcal{H}}\right|^{2}, \quad y \in \mathcal{H} .
$$

O teorema abaixo inclui no seu enunciado o Teorema de Riesz-Fischer ([12, p.175] e $[43$, p.34, 36]).

Teorema 1.4.4. Se $\mathcal{H}$ é um espaço de Hilbert e $\left\{x_{n}\right\}$ é uma base ortonormal de $\mathcal{H}$, então

$$
y=\sum_{n=1}^{\infty}\left\langle y, x_{n}\right\rangle_{\mathcal{H}} x_{n}, \quad y \in \mathcal{H}
$$

$e$

$$
\|y\|_{\mathcal{H}}^{2}=\sum_{n=1}^{\infty}\left|\left\langle y, x_{n}\right\rangle_{\mathcal{H}}\right|^{2}, \quad y \in \mathcal{H} .
$$

Além disso, se $\left\{c_{n}\right\}$ é uma seqüência de números complexos tal que $\sum_{n=1}^{\infty}\left|c_{n}\right|^{2}<\infty$, então $x:=\sum_{n=1}^{\infty} c_{n} x_{n} \in \mathcal{H}$ e $c_{n}=\left\langle x, x_{n}\right\rangle_{\mathcal{H}}$.

Exemplo 1.4.5. Se $X$ é um subconjunto mensurável de $\mathbb{R}^{m}$, então $L^{2}(X)$ é um espaço de Hilbert separável ([42, p.133]). 
Concluímos a seção com alguns resultados sobre tranformações lineares.

Definição 1.4.6. Sejam $\mathcal{X}$ e $\mathcal{Y}$ espaços vetoriais sobre $\mathbb{R}$ ou $\mathbb{C}$. O conjunto $\mathcal{L}(\mathcal{X}, \mathcal{Y})$ é o conjunto de todos os operadores lineares contínuos de $\mathcal{X}$ em $\mathcal{Y}$. Quando $X=Y$, escrevemos $\mathcal{L}(\mathcal{X}, \mathcal{Y})=\mathcal{L}(X)$.

A prova do resultado abaixo pode ser encontrada em [12, p.154].

Teorema 1.4.7. Valem as seguintes propriedades:

(i) Se $\mathcal{X}$ e $\mathcal{Y}$ são espaços vetoriais normados, então $\mathcal{L}(\mathcal{X}, \mathcal{Y})$ é um espaço vetorial normado. A expressão

$$
\begin{aligned}
\|T\|_{\mathcal{L}(\mathcal{X}, \mathcal{Y})} & :=\sup \left\{\|T(x)\|_{\mathcal{Y}}: x \in \mathcal{X},\|x\|_{\mathcal{X}}=1\right\} \\
& =\sup \left\{\frac{\|T(x)\|_{\mathcal{Y}}}{\|x\|_{\mathcal{X}}}: 0 \neq x \in \mathcal{X}\right\} \\
& =\inf \left\{C:\|T(x)\|_{\mathcal{Y}} \leq C\|x\|_{\mathcal{X}}, x \in \mathcal{X}\right\}
\end{aligned}
$$

define uma norma em $\mathcal{L}(\mathcal{X}, \mathcal{Y})$.

(ii) Nas condições em $(i)$, se $\mathcal{Y}$ é um espaço de Banach, então $\mathcal{L}(\mathcal{X}, \mathcal{Y})$ também o é.

A definição de operador adjunto origina-se do seguinte teorema ([43, p.76]).

Teorema 1.4.8. Sejam $\mathcal{H}_{1}$ e $\mathcal{H}_{2}$ espaços de Hilbert. Se $T \in \mathcal{L}\left(\mathcal{H}_{1}, \mathcal{H}_{2}\right)$, então existe um único operador $T^{*} \in \mathcal{L}\left(\mathcal{H}_{2}, \mathcal{H}_{1}\right)$ tal que

$$
\langle T(x), y\rangle_{\mathcal{H}_{2}}=\left\langle x, T^{*}(y)\right\rangle_{\mathcal{H}_{1}}, \quad x \in \mathcal{H}_{1}, \quad y \in \mathcal{H}_{2}
$$

Corolário 1.4.9. Sejam $\mathcal{H}$ um espaço de Hilbert e $T \in \mathcal{L}(\mathcal{H})$. Se existir um subconjunto ortonormal $\left\{x_{n}\right\}$ de $\mathcal{H}$ e uma seqüência limitada $\left\{\lambda_{n}\right\}$ tais que

$$
T(x)=\sum_{n=1}^{\infty} \lambda_{n}\left\langle x, x_{n}\right\rangle_{\mathcal{H}} x_{n},
$$

então

$$
T^{*}(x)=\sum_{n=1}^{\infty} \overline{\lambda_{n}}\left\langle x, x_{n}\right\rangle_{\mathcal{H}} x_{n} .
$$

Definição 1.4.10. O operador $T^{*}$ descrito no teorema anterior é denominado operador adjunto de $T$.

Definição 1.4.11. Sejam $\mathcal{H}$ um espaço de Hilbert e $T \in \mathcal{L}(\mathcal{H})$. Dizemos que $T$ é auto-adjunto quando $T=T^{*}$. Dizemos que $T$ é normal quando $T^{*} T=T T^{*}$. 
Definição 1.4.12. Sejam $\mathcal{X}$ e $\mathcal{Y}$ espaços de Banach. Um operador $T \in \mathcal{L}(\mathcal{X}, \mathcal{Y})$ é compacto ou completamente contínuo quando a imagem de cada seqüência limitada de $\mathcal{X}$ possui uma subseqüência convergente em $\mathcal{Y}$.

Um exemplo elementar de operador compacto é fornecido pelo teorema abaixo.

Teorema 1.4.13. Sejam $\mathcal{X}$ e $\mathcal{Y}$ espaços de Banach. Se $T \in \mathcal{L}(\mathcal{X}, \mathcal{Y})$ tem posto finito, então $T$ é compacto.

Demonstração: Seja $\left\{x_{n}\right\}$ uma seqüência em $\mathcal{X}$ e suponha que $\left\|x_{n}\right\|_{\mathcal{X}} \leq c<\infty$, $n=1,2, \ldots$, para algum $c>0$. Como $T$ tem posto finito, existe um subconjunto linearmente independente $\left\{y_{i}: i=1,2, \ldots, N\right\}$ de $\mathcal{Y}$ tal que

$$
T(x)=\sum_{i=0}^{N} \alpha_{i}(x) y_{i}, \quad x \in \mathcal{X} .
$$

Em particular, $T\left(x_{n}\right)=\sum_{i=0}^{N} \alpha_{i}\left(x_{n}\right) y_{i}$. Como $\left\|T\left(x_{n}\right)\right\|_{\mathcal{Y}} \leq c\|T\|_{\mathcal{L}(\mathcal{X}, \mathcal{Y})}<\infty, n=$ $1,2, \ldots$, a seqüência $\left\{v_{n}\right\}$ de $\mathbb{R}^{N}$ definida por $v_{n}:=\left(\alpha_{1}\left(x_{n}\right), \alpha_{2}\left(x_{n}\right), \ldots, \alpha_{N}\left(x_{n}\right)\right), n=$ $1,2 \ldots$, é limitada. Logo, possui uma subseqüência convergente. Portanto, $\left\{T\left(x_{n}\right)\right\}$ possui uma subseqüência convergente. A compacidade de $T$ segue.

Outra maneira de obtermos operadores compactos é sugerida pelo teorema abaixo ([33, p.200] e [40, p.124]).

Teorema 1.4.14. Sejam $\mathcal{X}, \mathcal{Y}$ e $\mathcal{Z}$ espaços de Banach. Se $T \in \mathcal{L}(\mathcal{X}, \mathcal{Y}), S \in \mathcal{L}(\mathcal{Y}, \mathcal{Z})$ e $T$ ou $S$ é compacto, então a composição ST é um operador compacto.

No contexto de espaços de Hilbert temos o seguinte resultado básico ([40, p.124]).

Teorema 1.4.15. Sejam $\mathcal{H}$ um espaço de Hilbert e $T \in \mathcal{L}(\mathcal{H})$. Então $T$ é um operador compacto se, e somente se, $T^{*}$ é compacto.

Definição 1.4.16. Seja $\mathcal{H}$ um espaço de Hilbert. Um operador $T \in \mathcal{L}(\mathcal{H})$ é positivo quando

$$
\langle T(x), x\rangle_{\mathcal{H}} \geq 0, \quad x \in \mathcal{H}
$$

Nas condições da definição acima, se $T \in \mathcal{L}(\mathcal{H})$ é positivo, escrevemos $T \geq 0$. Se $T_{1}, T_{2} \in \mathcal{L}(\mathcal{H})$, escrevemos $T_{1} \geq T_{2}$ para indicar que $T_{1}-T_{2} \geq 0$.

Se $T \in \mathcal{L}(\mathcal{H})$, então $T^{*} T \geq 0$ uma vez que

$$
\left\langle T^{*} T(x), x\right\rangle_{\mathcal{H}}=\overline{\left\langle x, T^{*} T(x)\right\rangle_{\mathcal{H}}}=\overline{\langle T(x), T(x)\rangle_{\mathcal{H}}}=\|T(x)\|_{\mathcal{H}}^{2} \geq 0, \quad x \in \mathcal{H} .
$$

Por outro lado, a identidade de polarização ([41, p.72]), revela que um operador positivo sobre um espaço de Hilbert complexo é auto-adjunto conforme o seguinte teorema. 
Teorema 1.4.17. Seja $\mathcal{H}$ um espaço de Hilbert complexo. Se $T \in \mathcal{L}(\mathcal{H})$ é um operador positivo, então T é um operador auto-adjunto.

Talvez, a principal característica de um operador positivo seja o fato do mesmo possuir uma única raíz quadrada ([43, p.221]).

Teorema 1.4.18 (Lema da Raiz Quadrada). Sejam $\mathcal{H}$ um espaço de Hilbert e $T \in \mathcal{L}(\mathcal{H})$. Se $T \geq 0$, então existe um único $\mathcal{S} \in \mathcal{L}(\mathcal{H}), \mathcal{S} \geq 0$, tal que $\mathcal{S}^{2}=T$.

O operador $\mathcal{S}$ descrito acima é usualmente denotado por $\sqrt{T}$ e chamado de raíz quadrada de $T$.

Se $T \in \mathcal{L}(\mathcal{H})$, definimos $|T|:=\sqrt{T^{*} T}$. Observe que se $T \geq 0$, então $|T|=T$. O teorema abaixo fornece uma caracterização de compacidade via este conceito ([40, p.124]).

Teorema 1.4.19. Sejam $\mathcal{H}$ um espaço de Hilbert e $T \in \mathcal{L}(\mathcal{H})$. Então $T$ é um operador compacto se, e somente se, $|T|$ é um operador compacto.

Definição 1.4.20. Sejam $\mathcal{H}$ um espaço de Hilbert e $\left\{x_{n}\right\}$ uma base ortonormal de $\mathcal{H}$. Se $T \in \mathcal{L}(\mathcal{H})$ e $T \geq 0$, o traço de $T$ é definido por

$$
\operatorname{tr}(T):=\sum_{n=1}^{\infty}\left\langle T\left(x_{n}\right), x_{n}\right\rangle_{\mathcal{H}} .
$$

Não é difícil verificar que $\operatorname{tr}(T)$ independe da base ortonormal utilizada ([33, p.206]). Fechamos a seção introduzindo mais uma categoria de operadores que utilizamos no trabalho.

Definição 1.4.21. Seja $\mathcal{H}$ espaço de Hilbert. Um operador $T \in \mathcal{L}(\mathcal{H})$ é nuclear quando $\operatorname{tr}(|T|):=\operatorname{tr}\left(\sqrt{T^{*} T}\right)<\infty$.

A nomenclatura em inglês para o termo nuclear utilizado acima é "trace-class". Como não encontramos uma tradução para o termo, preferimos usar esta que é mais comum no contexto de espaços de Banach.

As propriedades básicas dos operadores nucleares que utilizamos estão listadas abaixo ([33, p.207, 209, 211]).

Teorema 1.4.22. Nas condições da definição acima, valem as seguintes propriedades:

(i) O conjunto dos operadores nucleares é um sub-espaço vetorial de $\mathcal{L}(\mathcal{H})$;

(ii) Se $T \in \mathcal{L}(\mathcal{H})$ é um operador nuclear e $\left\{x_{n}\right\}$ é uma base ortonormal de $\mathcal{H}$, então a série $\sum_{n=1}^{\infty}\left\langle T\left(x_{n}\right), x_{n}\right\rangle_{\mathcal{H}}$ é absolutamente convergente;

(iii) Nas condições do item (ii), o valor da série independe da base utilizada;

(iv) Se $T \in \mathcal{L}(\mathcal{H})$ é nuclear, então $T$ é compacto. 
O espaço dos operadores nucleares é normalizável. Uma possível norma é dada pela expressão

$$
\|T\|_{t r}:=\sum_{n=1}^{\infty}\left|\left\langle T\left(x_{n}\right), x_{n}\right\rangle_{\mathcal{H}}\right|,
$$

onde $\left\{x_{n}\right\}$ é uma base ortonormal de $\mathcal{H}$. Como a expressão $\operatorname{tr}(T):=\sum_{n=1}^{\infty}\left\langle T\left(x_{n}\right), x_{n}\right\rangle_{\mathcal{H}}$ é absolutamente convergente e independe da base é imediato que $\operatorname{tr}(\cdot)$ é um funcional linear contínuo no espaço dos operadores nucleares com norma menor ou igual a 1.

\subsection{Um pouco de teoria espectral}

Esta seção contém aqueles resultados da Teoria Espectral que envolvem operadores dos tipos citados na seção anterior.

No caso de operadores compactos sobre espaços de Hilbert, o resultado mais básico é o Teorema de Riesz-Schauder ([33, p.203]). Utilizamos o símbolo $\sigma(T)$ para denotar o espectro de um operador $T$.

Teorema 1.5.1 (Riesz-Schauder). Seja $\mathcal{H}$ um espaço de Hilbert. Se T é um operador compacto sobre $\mathcal{H}$, então $\sigma(T)$ é um subconjunto discreto de $\mathbb{C}$, sem pontos de acumulação, exceto possivelmente 0. Além disso, $\lambda(T) \in \sigma(T) \backslash\{0\}$ é um autovalor de $T$ de multiplicidade finita.

Quando o operador é também auto-adjunto, o resultado anterior pode ser refinado como segue ([33, p.203]). Por simplicidade de notação a utilizamos o símbolo $\lambda(T)$ para representar um autovalor do operador $T$ ou 0 o qual também será chamado de autovalor, sem prejuísos aos resultados.

Teorema 1.5.2 (Hilbert-Schmidt). Seja T um operador compacto sobre um espaço de Hilbert $\mathcal{H}$. Se $T$ é auto-adjunto, então existe uma base ortonormal $\left\{x_{n}\right\}$ de $\mathcal{H} e$ $\left\{\lambda_{n}(T)\right\} \subset \mathbb{R}$ tais que

$$
T(x)=\sum_{n=1}^{\infty} \lambda_{n}(T)\left\langle x, x_{n}\right\rangle_{\mathcal{H}} x_{n}, \quad x \in \mathcal{H},
$$

com $\left|\lambda_{1}(T)\right| \geq\left|\lambda_{2}(T)\right| \geq \cdots \geq 0$ e $\lim _{n \rightarrow \infty} \lambda_{n}(T)=0$. A convergência da série é na norma de $\mathcal{H}$.

Uma versão do teorema no caso em que o operador é compacto e normal pode ser encontrada em [41, p.167].

Corolário 1.5.3. Nas condições do Teorema de Hilbert-Schmidt, se $T \geq 0$, então $\lambda_{n}(T) \geq 0, n=1,2, \ldots$ 
Demonstração: Basta notar que se $T(x)=\lambda(T) x$ e $x \neq 0$, então $0 \leq\langle T(x), x\rangle_{\mathcal{H}}=$ $\lambda(T)\langle x, x\rangle_{\mathcal{H}}$.

Se $T$ é um operador compacto sobre um espaço de Hilbert, já sabemos que $|T|$ é auto-adjunto e positivo. Logo, o Teorema de Hilbert-Schmidt é aplicável para este operador. Por simplicidade, denotamos os autovalores de $|T|$ por $\left\{s_{n}(T)\right\}$ e assumimos que os mesmos estão ordenados na forma

$$
s_{1}(T) \geq s_{2}(T) \geq \cdots \geq 0
$$

levando-se em conta possíveis repetições relacionadas com a multiplicidade algébrica de $s_{j}(T)$.

Utilizando-se o teorema anterior e algumas manipulações algébricas, não é difícil verificar que se $T$ é compacto e auto-adjunto, ou pelo menos normal, sobre um espaço de Hilbert, então $\lambda_{n}\left(T^{*}\right)=\overline{\lambda_{n}(T)}$ e $s_{n}(T)=\left|\lambda_{n}(T)\right|([15, \mathrm{p} .27])$.

$\mathrm{Na}$ análise do decaimento dos autovalores de operadores compactos, alguns resultados mais finos de análise espectral são necessários. Um deles é descrito abaixo ([14, p.51] e $[15$, p.28]).

Teorema 1.5.4. Se $\mathcal{H}$ é um espaço de Hilbert e $T \in \mathcal{L}(\mathcal{H})$ é compacto, então

$$
s_{n+1}(T)=\min \left\{\|T-S\|_{\mathcal{L}(\mathcal{H})}: S \in \mathrm{F}_{n}\right\}, \quad n=1,2, \ldots
$$

onde $\mathrm{F}_{n}$ é o subconjunto de $\mathcal{L}(\mathcal{H})$ formado pelos operadores de posto no máximo $n$.

\subsection{Operadores do tipo Hilbert-Schmidt}

Muitos resultados obtidos nas seções anteriores são válidos quando o operador em questão é compacto. Nesta seção estudamos algumas propriedades de uma classe especial de operadores compactos, os operadores do tipo Hilbert-Schmidt.

Definição 1.6.1. Seja $\mathcal{H}$ um espaço de Hilbert. Dizemos que um operador $T \in \mathcal{L}(\mathcal{H})$ é do tipo Hilbert-Schmidt quando $T^{*} T$ é nuclear.

Algumas propriedades básicas dos operadores do tipo Hilbert-Schmidt estão registradas no teorema abaixo ([33, p.210]).

Teorema 1.6.2. Seja $\mathcal{H}$ um espaço de Hilbert.

(i) O conjunto $\mathfrak{F}$ formado pelos operadores do tipo Hilbert-Schmidt sobre $\mathcal{H}$ é um espaço vetorial. A expressão

$$
\|T\|_{\mathfrak{F}}:=\sqrt{\operatorname{tr}\left(T^{*} T\right)}, \quad T \in \mathfrak{F}
$$


define uma norma em $\mathfrak{F}$;

(ii) $S e T \in \mathcal{L}(\mathcal{H})$ é do tipo Hilbert-Schmidt, então $T$ é compacto;

(iii) $\operatorname{Se} T \in \mathcal{L}(\mathcal{H})$ é do tipo Hilbert-Schmidt, então $\|T\|_{\mathcal{L}(\mathcal{H})} \leq\|T\|_{\mathfrak{F}}$.

Concluímos a seção apresentando uma caracterização para operadores do tipo HilbertSchmidt. A prova pode ser encontrada em [33, p. 210] e [40, p.132].

Teorema 1.6.3. Sejam $(M, \mathcal{M}, \mu)$ um espaço de medida $\sigma$-finito para o qual $\mathcal{H}=$ $L^{2}(M, \mu)$ é separável e $T \in \mathcal{L}(\mathcal{H})$. As seguintes afirmações são equivalentes:

(i) $T$ é do tipo Hilbert-Schmidt;

(ii) Existe $K \in L^{2}(M \times M, \mu \times \mu)$ tal que

$$
T(f)(x)=\int_{M} K(x, y) f(y) d \mu(y), \quad f \in \mathcal{H}(q . s .)
$$

Observe que no caso de $T$ ser do tipo Hilbert-Schmidt, a representação de $T$ dada pelo teorema acima, ainda nos dá a seguinte fórmula:

$$
\|T\|_{\mathcal{L}(\mathcal{H})}^{2} \leq\|T\|_{\mathfrak{F}}^{2}=\operatorname{tr}\left(T^{*} T\right)=\int_{M \times M}|K(x, y)|^{2} d \mu(x) d \mu(y) .
$$

Observação 1.6.4. Operadores com uma representação como aquela descrita no item (ii) do teorema anterior são comumente chamados de operadores integrais. Na formulação mais comum deste trabalho eles são da forma $T: L^{2}(X) \rightarrow L^{2}(X)$, onde $X \subset \mathbb{R}^{m}$ é Lebesgue-mensurável. Neste caso, sempre escreveremos $T=\mathcal{K}$ em consonância com a letra $K$ usada para indicar a função geradora do operador. Se $K \in L^{2}(X \times X)$, a desigualdade de Cauchy-Schwarz e o Teorema de Fubini implicam que

$$
\begin{aligned}
\|\mathcal{K}(\phi)\|_{L^{2}(X)}^{2} & =\int_{X}|\mathcal{K}(\phi)(x)|^{2} d x \\
& =\int_{X}\left|\int_{X} K(x, y) \phi(y) d y\right|^{2} d x \\
& \leq \int_{X}\left(\left(\int_{X}|K(x, y)|^{2} d y\right)^{1 / 2}\left(\int_{X}|\phi(y)|^{2} d y\right)^{1 / 2}\right)^{2} d x \\
& =\|K\|_{L^{2}(X \times X)}^{2}\|\phi\|_{L^{2}(X)}^{2}, \quad \phi \in L^{2}(X)
\end{aligned}
$$

ou seja, $\|\mathcal{K}\|_{\mathcal{L}\left(L^{2}(X)\right)} \leq\|K\|_{L^{2}(X \times X)}$.

A inspiração para o estudo da representação de núcleos positivos definidos por séries que desenvolveremos no Capítulo 2 reside no Teorema 1.6.6. O lema abaixo é técnico e facilita a demonstração do teorema. 
Lema 1.6.5. Sejam $\left(M_{1}, \mathcal{M}_{1}, \mu_{1}\right)$ e $\left(M_{2}, \mathcal{M}_{2}, \mu_{2}\right)$ espaços de medida $\sigma$-finitos. Suponha que $L^{2}\left(M_{1}, \mu_{1}\right)$ e $L^{2}\left(M_{2}, \mu_{2}\right)$ são espaços separáveis e sejam $\left\{\phi_{n}\right\}$ e $\left\{\psi_{m}\right\}$ bases ortonormais de $L^{2}\left(M_{1}, \mu_{1}\right)$ e $L^{2}\left(M_{2}, \mu_{2}\right)$, respectivamente. Defina

$$
\phi_{n} \otimes \psi_{m}(x, y):=\phi_{n}(x) \psi_{m}(y), \quad m, n=1,2, \ldots, \quad x \in M_{1}, \quad y \in M_{2} .
$$

Então $\left\{\phi_{n} \otimes \psi_{m}: m, n=1,2, \ldots\right\}$ é uma base ortonormal de $L^{2}\left(M_{1} \times M_{2}, \mu_{1} \times \mu_{2}\right)$.

Demonstração: Não é difícil ver que a seqüência dupla $\left\{\phi_{n} \otimes \psi_{m}\right\}$ é um subconjunto ortonormal de $L^{2}\left(M_{1} \times M_{2}, \mu_{1} \times \mu_{2}\right)$. Mostremos que é uma base ortonormal. Suponha que $f \in L^{2}\left(M_{1} \times M_{2}, \mu_{1} \times \mu_{2}\right)$ é ortogonal a este conjunto, ou seja, que

$$
\int_{M_{2}} \int_{M_{1}} f(x, y) \overline{\phi_{n}(x) \psi_{m}(y)} d \mu_{1}(x) d \mu_{2}(y)=0, \quad m, n=1,2, \ldots
$$

Aplicando o Teorema de Fubini, temos que

$$
\int_{M_{2}}\left[\int_{M_{1}} f(x, y) \overline{\phi_{n}(x)} d \mu_{1}(x)\right] \overline{\psi_{m}(y)} d \mu_{2}(y)=0 .
$$

Como a integral entre colchetes na expressão acima define um elemento de $L^{2}\left(M_{2}, \mu_{2}\right)$ e $\left\{\psi_{m}\right\}$ é uma base de $L^{2}\left(M_{2}, d \mu_{2}\right)$, temos que

$$
\int_{M_{1}} f(x, y) \overline{\phi_{n}(x)} d \mu_{1}(x)=0
$$

exceto para $y \in S_{n} \subset M_{2}$, onde $\mu_{2}\left(S_{n}\right)=0$. Segue que

$$
\int_{M_{1}} f(x, y) \overline{\phi_{n}(x)} d \mu_{1}(x)=0, \quad n=1,2, \ldots, \quad y \in M_{2} \backslash \cup_{n=1}^{\infty} S_{n} .
$$

conseqüentemente, para cada $y \in M_{2} \backslash \cup_{n=1}^{\infty} S_{n}, f(x, y)=0 \mu_{1}$-quase sempre, pois $\left\{\phi_{n}\right\}$ é base de $L^{2}\left(M_{1}, \mu_{1}\right)$ e $f(., y) \in L^{2}\left(M_{1}, \mu_{1}\right)$ quase sempre. Como $\mu_{2}\left(\cup_{n=1}^{\infty} S_{n}\right)=0$, $f(x, y)=0 \mu_{1} \times \mu_{2}$-quase sempre. Portanto, a prova está completa.

Deste ponto em diante, toda função da forma $K: X \times X \rightarrow \mathbb{C}$ será chamada de núcleo sobre $X$, ou simplesmente núcleo, quando o contexto permitir. No teorema abaixo utilizamos a noção de núcleo hermitiano, significando que $K(x, y)=\overline{K(y, x)}$, $x, y \in X$.

Teorema 1.6.6. Sejam $(X, \mathcal{M}, \mu)$ um espaço de medida $\sigma$-finito e $K \in L^{2}(X \times X)$. Se $L^{2}(X)$ é separável e $K$ é hermitiano então existe uma seqüência $\left\{\lambda_{n}\right\}:=\left\{\lambda_{n}(\mathcal{K})\right\} \subset \mathbb{R}$ e uma base ortonormal $\left\{\phi_{n}\right\}$ de $L^{2}(X)$ tais que

$$
K=\sum_{n=1}^{\infty} \lambda_{n}(\mathcal{K}) \phi_{n} \otimes \overline{\phi_{n}}
$$

A convergência da série é em $L^{2}(X \times X)$. 
Demonstração: Como $K$ é hermitiano, o operador integral associado $\mathcal{K}$ é autoadjunto. Sendo um operador integral, o Teorema 1.6.3 garante que $\mathcal{K}$ é do tipo HilbertSchmidt, logo compacto. Pelo Teorema 1.5.2 segue que existe uma base ortonormal $\left\{\phi_{n}\right\}$ de $L^{2}(X)$ e uma seqüência $\left\{\lambda_{n}(\mathcal{K})\right\} \subset \mathbb{R}$ tais que $\mathcal{K}\left(\phi_{n}\right)=\lambda_{n}(\mathcal{K}) \phi_{n}$. Como o Lema 1.6.5 garante que $\left\{\phi_{n} \otimes \overline{\phi_{m}}\right\}$ é uma base ortonormal de $L^{2}(X \times X)$, podemos usar o Teorema 1.4.4, para escrever

$$
K=\sum_{m, n=1}^{\infty}\left\langle K, \phi_{n} \otimes \overline{\phi_{m}}\right\rangle \phi_{n} \otimes \overline{\phi_{m}} .
$$

Entretanto, pelo Teorema de Fubini,

$$
\begin{aligned}
\left\langle K, \phi_{n} \otimes \overline{\phi_{m}}\right\rangle & =\int_{X} \int_{X} K(x, y) \overline{\phi_{n}(x)} \phi_{m}(y) d \mu(x) d \mu(y) \\
& =\int_{X}\left[\int_{X} K(x, y) \phi_{m}(y) d \mu(y)\right] \overline{\phi_{n}(x)} d \mu(x) \\
& =\int_{X} \lambda_{m}(\mathcal{K}) \phi_{m}(x) \overline{\phi_{n}(x)} d \mu(x) \\
& =\lambda_{m}(\mathcal{K}) \delta_{m n},
\end{aligned}
$$

onde $\delta_{m n}$ é a função delta de Kronecker. Segue que

$$
K=\sum_{n=1}^{\infty} \lambda_{n}(\mathcal{K}) \phi_{n} \otimes \overline{\phi_{n}}
$$

e também que a convergência é em $L^{2}(X \times X)$.

O lema seguinte será útil posteriormente.

Lema 1.6.7. Sejam $\left(M_{1}, \mathcal{M}_{1}, \mu_{1}\right)$ e $\left(M_{2}, \mathcal{M}_{2}, \mu_{2}\right)$ espaços de medida $\sigma$-finitos, $\left\{f_{n}\right\} \subset$ $L^{2}\left(M_{1}, d \mu_{1}\right)$ e $\left\{g_{n}\right\} \subset L^{2}\left(M_{2}, d \mu_{2}\right)$. Assuma que existem $f \in L^{2}\left(M_{1}, d \mu_{1}\right)$ e $g \in$ $L^{2}\left(M_{2}, d \mu_{2}\right)$ tais que $\lim _{n \rightarrow \infty}\left\|f_{n}-f\right\|_{L^{2}\left(M_{1}, \mu_{1}\right)}=0$ e $\lim _{n \rightarrow \infty}\left\|g_{n} \rightarrow g\right\|_{L^{2}\left(M_{2}, \mu_{2}\right)}=0$. Então

$$
\lim _{n \rightarrow \infty}\left\|f_{n} \otimes g_{n}-f \otimes g\right\|_{L^{2}\left(M_{1} \times M_{2}, \mu_{1} \times \mu_{2}\right)}=0 .
$$

Demonstração: Por simplicidade vamos omitir os sub-índices nas normas. Inicialmente note que

$$
\begin{aligned}
\left\|f_{n} \otimes g_{n}-f \otimes g\right\| & =\left\|f_{n} \otimes g_{n}-f_{n} \otimes g+f_{n} \otimes g-f \otimes g\right\| \\
& \leq\left\|f_{n} \otimes g_{n}-f_{n} \otimes g\right\|+\left\|f_{n} \otimes g-f \otimes g\right\| \\
& =\left\|f_{n} \otimes\left(g_{n}-g\right)\right\|+\left\|\left(f_{n}-f\right) \otimes g\right\|, \quad n=1,2, \ldots .
\end{aligned}
$$

Aplicando o Teorema de Fubini, concluímos que

$$
\left\|f_{n} \otimes g_{n}-f \otimes g\right\| \leq\left\|f_{n}\right\|\left\|g_{n}-g\right\|+\left\|f_{n}-f\right\|\|g\|, \quad n=1,2, \ldots,
$$

e o resultado segue. 


\subsection{Transformada de Fourier}

Nesta seção apresentamos muito rapidamente o conceito de transformada de Fourier e registramos algumas de suas propriedades. As demonstrações e resultados mais delicados relativos ao conceito podem ser encontrados em [12] e [13]. O produto interno usual de dois vetores $x, y$ de $\mathbb{R}^{m}$ é denotado por $x \cdot y$.

Definição 1.7.1. A transformada de Fourier é a função $f \in L^{1}\left(\mathbb{R}^{m}\right) \mapsto \mathcal{F}(f)$ dada pela fórmula

$$
\mathcal{F}(f)(v)=\int_{\mathbb{R}^{m}} f(x) e^{-2 \pi i x \cdot v} d x, \quad v \in \mathbb{R}^{m}
$$

Em geral escrevemos: $\hat{f}:=\mathcal{F}(f)$. Como

$$
|\hat{f}(v)| \leq \int_{\mathbb{R}^{m}}\left|f(x) e^{-2 \pi i x \cdot v}\right| d x=\int_{\mathbb{R}^{m}}|f(x)| d x=\|f\|_{L^{1}\left(\mathbb{R}^{m}\right)}, \quad v \in \mathbb{R}^{m},
$$

$\hat{f}$ é uma função limitada. Ainda, se $f, g \in L^{1}\left(\mathbb{R}^{m}\right)$ e $\alpha \in \mathbb{C}$, então

$$
(\widehat{f+\alpha g})(v)=\hat{f}(v)+\alpha \hat{g}(v), \quad v \in \mathbb{R}^{m}
$$

ou seja, a transformada de Fourier é linear.

A definição de transformada de Fourier também se aplica às funções pertencentes a $L^{1}\left(\mathbb{R}^{m}\right) \cap L^{2}\left(\mathbb{R}^{m}\right)$, que é um subconjunto denso de $L^{2}\left(\mathbb{R}^{m}\right)$. O teorema a seguir ([12, p.253]) permite a extensão do conceito para todas as funções deste espaço. Entretanto, ele é apenas um teorema de existência, não dando uma expressão geral para a transformada de Fourier.

Teorema 1.7.2 (Plancherel). Se $f \in L^{1}\left(\mathbb{R}^{m}\right) \cap L^{2}\left(\mathbb{R}^{m}\right)$, então $\mathcal{F}(f) \in L^{2}\left(\mathbb{R}^{m}\right)$. Ainda, a restrição de $\mathcal{F}$ a $L^{1}\left(\mathbb{R}^{m}\right) \cap L^{2}\left(\mathbb{R}^{m}\right)$ pode ser estendida, de maneira única, a um isomorfismo unitário de $L^{2}\left(\mathbb{R}^{m}\right)$ em $L^{2}\left(\mathbb{R}^{m}\right)$.

Corolário 1.7.3 (Identidade de Parseval). Se $f, g \in L^{2}\left(\mathbb{R}^{m}\right)$, então

$$
\langle f, g\rangle=\int_{\mathbb{R}^{m}} f(x) \overline{g(x)} d x=\int_{\mathbb{R}^{m}} \hat{f}(x) \overline{\hat{g}(x)} d x=\langle\hat{f}, \hat{g}\rangle .
$$

Para finalizar a seção, apresentamos três resultados que utilizamos à frente.

Lema 1.7.4 (Riemann-Lebesgue). Se $f \in L^{1}\left(\mathbb{R}^{m}\right)$, então $\hat{f}$ é contínua e possui o comportamento assintótico $\lim _{|v| \rightarrow \infty} \hat{f}(v)=0$. Em particular, $\hat{f}$ é uniformemente continua.

Lema 1.7.5. Se $f \in L^{2}\left(\mathbb{R}^{m}\right)$, então $\hat{f(v)}=\overline{\hat{f}(-v)}, v \in \mathbb{R}^{m}$. 
Demonstração: Se $\phi \in L^{1}\left(\mathbb{R}^{m}\right) \cap L^{2}\left(\mathbb{R}^{m}\right)$, então

$$
\hat{\overline{\phi(v)}}=\int_{\mathbb{R}^{m}} \overline{\phi(x)} e^{-2 \pi i x \cdot v} d x=\overline{\int_{\mathbb{R}^{m}} \phi(x) e^{-2 \pi i x \cdot(-v)} d x}=\overline{\hat{\phi}(-v)}, \quad v \in \mathbb{R}^{m} .
$$

Se $f \in L^{2}\left(\mathbb{R}^{m}\right)$, usamos a densidade de $L^{1}\left(\mathbb{R}^{m}\right) \cap L^{2}\left(\mathbb{R}^{m}\right)$ em $L^{2}\left(\mathbb{R}^{m}\right)$ e o Teorema 1.3.8 para obter uma seqüência $\left\{f_{n}\right\} \subset L^{1}\left(\mathbb{R}^{m}\right) \cap L^{2}\left(\mathbb{R}^{m}\right)$ tal que $f_{n} \rightarrow f$ em $L^{2}\left(\mathbb{R}^{m}\right)$ e $f_{n} \rightarrow f$ quase sempre. Como a transformada de Fourier estendida é um isomorfismo, temos que $\hat{f}_{n} \rightarrow \hat{f}$ em $L^{2}\left(\mathbb{R}^{m}\right)$ e podemos assumir que esta convergência é tambem quase sempre. Conseqüentemente,

$$
\lim _{n \rightarrow \infty} \overline{f_{n}(v)}=\lim _{n \rightarrow \infty} \overline{\hat{f}_{n}(-v)}=\hat{f(v)}=\overline{\hat{f}(-v)}, \quad v \in \mathbb{R}^{m} \text { q.s. }
$$

Redefinindo-se $\hat{f}$ em um conjunto de medida nula, se necessário, o resultado segue.

Observação 1.7.6. Para funções de $L^{p}\left(R^{m} \times \mathbb{R}^{m}\right), p=1,2$, vamos escrever convenientemente a transformada de Fourier. Para tanto, note que podemos visualizar uma função $f(x, y), x, y \in \mathbb{R}^{m}$ como uma função $g(z)$, onde $z=\left(x_{1}, \ldots, x_{m}, y_{1}, \ldots, y_{m}\right) \in$ $\mathbb{R}^{2 m}$. Com esta observação é natural que a transformada de Fourier de $f \in L^{1}\left(R^{m} \times \mathbb{R}^{m}\right)$ seja definida, via Teorema de Fubini, da seguinte forma

$$
\hat{f}(v, w)=\int_{\mathbb{R}^{m}} \int_{\mathbb{R}^{m}} f(x, y) e^{-2 \pi i(x, y) \cdot(v, w)} d x d y=\int_{\mathbb{R}^{2 m}} g(z) e^{-2 \pi i z \cdot u} d z=\hat{g}(z),
$$

onde $u=\left(v_{1}, \ldots, v_{m}, w_{1}, \ldots, w_{m}\right)$.

Lema 1.7.7. Se $\phi, \psi \in L^{2}\left(\mathbb{R}^{m}\right)$, então $\widehat{\phi \otimes \psi}(v, w)=\hat{\phi}(v) \hat{\psi}(w), v, w \in \mathbb{R}^{m}$.

Demonstração: Se $\phi, \psi \in L^{2}\left(\mathbb{R}^{m}\right)$, existem seqüências $\left\{\phi_{n}\right\}$ e $\left\{\psi_{n}\right\}$ de $L^{1}\left(\mathbb{R}^{m}\right) \cap$ $L^{2}\left(\mathbb{R}^{m}\right)$ que convergem, em $L^{2}\left(\mathbb{R}^{m}\right)$, para $\phi$ e $\psi$, respectivamente. Logo, pelo Lema 1.6.7, a seqüência $\left\{\phi_{n} \otimes \psi_{n}\right\}$ converge para $\phi \otimes \psi$, em $L^{2}\left(\mathbb{R}^{m} \times \mathbb{R}^{m}\right)$. Como $\phi_{n} \otimes \psi_{n} \in$ $L^{1}\left(\mathbb{R}^{m} \times \mathbb{R}^{m}\right) \cap L^{2}\left(\mathbb{R}^{m} \times \mathbb{R}^{m}\right), n=1,2, \ldots$,

$$
\widehat{\phi_{n} \otimes \psi_{n}}(v, w)=\hat{\phi}_{n}(v) \hat{\psi}_{n}(w), \quad v, w \in \mathbb{R}^{m}, \quad n=1,2, \ldots
$$

Tomando-se o limite quando $n \rightarrow \infty$ e usando a continuidade da transformada de Fourier, deduzimos que $\widehat{\phi \otimes \psi}(v, w)=\hat{\phi}(v) \hat{\psi}(w), v, w \in \mathbb{R}^{m}$, quase sempre. Redefinindo $\widehat{\phi \otimes \psi}$ em um conjunto de medida nula, se necessário, concluímos a prova do lema. 


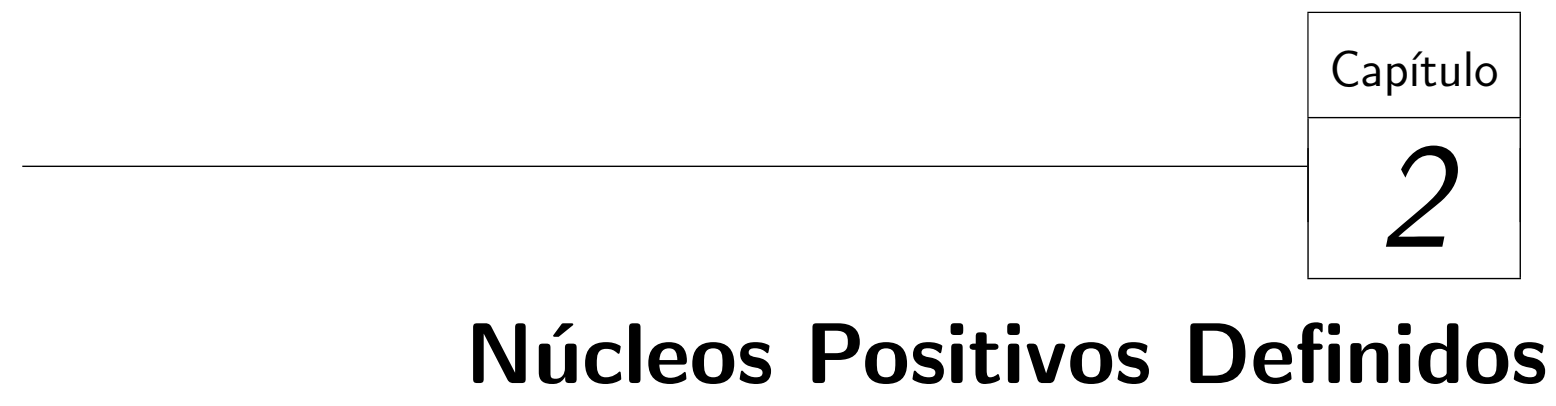

Núcleos Positivos Definidos

Neste capítulo apresentamos duas vertentes para o conceito de positividade definida de um núcleo $K: X \times X \rightarrow \mathbb{C}$. Estudamos possíveis relações entre os conceitos e algumas de suas propriedades. Apresentamos algumas variações e generalizações e finalizamos estudando algumas propriedades da transformada de Fourier de um núcleo positivo definido, no caso em que $X=\mathbb{R}^{m}$.

\subsection{Núcleos positivos definidos}

Nesta seção introduzimos o conceito usual de positividade definida e analisamos algumas de suas propriedades.

Definição 2.1.1. Seja $X$ um conjunto não-vazio. Dizemos que um núcleo $K$ sobre $X$ é positivo definido quando a matriz $A=\left(K\left(x_{i}, x_{j}\right)\right)$ de ordem n é não-negativa definida, para qualquer $n \geq 1$ e qualquer $n$-upla $\left(x_{1}, x_{2}, \ldots, x_{n}\right) \in X^{n}$.

É fácil ver que a definição acima equivale então à validade da desigualdade

$$
\sum_{i, j=1}^{n} \overline{c_{i}} c_{j} K\left(x_{i}, x_{j}\right) \geq 0,
$$

quando $n \geq 1,\left\{x_{1}, x_{2}, \ldots, x_{n}\right\} \subset X$ e $\left\{c_{1}, c_{2}, \ldots, c_{n}\right\} \subset \mathbb{C}$. Escrevemos $P D(X)$ para denotar a classe dos núcleos positivos definidos com domínio $X \times X$.

Exemplo 2.1.2. Se $f: X \rightarrow \mathbb{C}$ é uma função qualquer, o núcleo dado pela fórmula $K:=f \otimes \bar{f}$, é positivo definido. De fato, se $\left\{x_{1}, x_{2}, \ldots, x_{n}\right\} \subset X$ e $\left\{c_{1}, c_{2}, \ldots, c_{n}\right\} \subset \mathbb{C}$, 
então

$$
\sum_{i, j=1}^{n} \overline{c_{i}} c_{j} K\left(x_{i}, x_{j}\right)=\sum_{i, j=1}^{n} \overline{c_{i}} c_{j} f\left(x_{i}\right) \overline{f\left(x_{j}\right)}=\sum_{i, j=1}^{n} \overline{c_{i}} f\left(x_{i}\right) \overline{\overline{c_{j}} f\left(x_{j}\right)}=\left|\sum_{i, j=1}^{n} \overline{c_{i}} f\left(x_{i}\right)\right|^{2} .
$$

Exemplo 2.1.3. Se $X$ é um espaço vetorial complexo com produto interno $\langle\cdot, \cdot\rangle_{X}$, então $K(x, y):=\langle x, y\rangle_{X}, x, y \in X$, é positivo definido. Basta notar que a forma quadrática da definição pode ser escrita na forma

$$
\sum_{i, j=1}^{n} \overline{c_{i}} c_{j} K\left(x_{i}, x_{j}\right)=\left\|\sum_{j=1}^{n} \overline{c_{j}} x_{j}\right\|_{X}^{2}
$$

Exemplo 2.1.4. Seja $(X, \mu)$ um espaço de medida e $S: X \times X \rightarrow \mathbb{C}$ tal que $S(\cdot, x) \in$ $L^{2}(X), x \in X$. Neste caso o núcleo $K$ dado por

$$
K(x, y)=\int_{X} S(z, y) \overline{S(z, x)} d \mu(z), \quad x \in X,
$$

é um elemento de $P D(X)$. De fato, a forma quadrática da definição toma a forma

$$
\sum_{i, j=1}^{n} \bar{c}_{i} c_{j} K\left(x_{i}, x_{j}\right)=\int_{X} \sum_{i, j=1}^{n} \overline{c_{i}} c_{j} S\left(z, x_{j}\right) \overline{S\left(z, x_{i}\right)} d \mu(z)=\int_{X}\left|\sum_{j=1}^{n} c_{j} S\left(z, x_{j}\right)\right|^{2} d \mu(z) .
$$

O exemplo anterior nos dá uma fórmula para a construção de núcleos positivos definidos. Veremos posteriormente (veja o Lema 3.3.3) que alguns núcleos positivos definidos de $L^{2}(X \times X)$ que geram operadores integrais nucleares possuem uma representação semelhante.

Listamos abaixo algumas propriedades importantes dos núcleos positivos definidos.

\section{Propriedades 2.1.5.}

(i) Se $K_{1}, K_{2}, \ldots, K_{p} \in P D(X)$ e $d_{1}, d_{2}, \ldots, d_{p} \geq 0$, então $\sum_{j=1}^{p} d_{j} K_{j} \in P D(X)$.

De fato, basta observar que a forma quadrática para $K$ toma a forma

$$
\sum_{i, j=1}^{n} \overline{c_{i}} c_{j} K\left(x_{i}, x_{j}\right)=\sum_{l=1}^{p} d_{l}\left(\sum_{i, j=1}^{n} \overline{c_{i}} c_{j} K_{l}\left(x_{i}, x_{j}\right)\right) .
$$

(ii) Se $\left\{K_{p}\right\}$ é uma seqüência em $P D(X)$ que converge para $K$, então $K \in P D(X)$. De fato, neste caso a forma quadrática para $K$ toma a forma

$$
\sum_{i, j=1}^{n} \overline{c_{i}} c_{j} K\left(x_{i}, x_{j}\right)=\lim _{p \rightarrow \infty} \sum_{i, j=1}^{n} \overline{c_{i}} c_{j} K_{p}\left(x_{i}, x_{j}\right)
$$

(iii) Se $K_{1}, K_{2} \in P D(X)$, então o produto $K_{1} K_{2} \in P D(X)$.

De fato, isso segue diretamente do Teorema do Produto de Schür ([17, p.79]). 
(iv) Se $K \in P D(X)$ é limitado, $f(z)=\sum_{n=0}^{\infty} a_{n} z^{n}$ é holomorfa em $\{z \in \mathbb{C}:|z|<\rho\}$, $a_{n} \geq 0, n=0,1, \ldots$, e a composição $f \circ K$ faz sentido, então $f \circ K \in P D(X)$.

(v) Seja $K: X \times X \rightarrow \mathbb{R}$ um núcleo hermitiano. Então $K \in P D(X)$ se, e somente se,

$$
\sum_{l, j=1}^{n} c_{l} c_{j} K\left(x_{l}, x_{j}\right) \geq 0
$$

quando $n \geq 1,\left\{x_{1}, x_{2}, \ldots, x_{n}\right\} \subset X$ e $\left\{c_{1}, c_{2}, \ldots, c_{n}\right\} \subset \mathbb{R}$.

Basta notar que, se $\left\{x_{1}, x_{2}, \ldots, x_{n}\right\} \subset X,\left\{c_{1}, c_{2}, \ldots, c_{n}\right\} \subset \mathbb{C}$ e $c_{j}=a_{j}+i b_{j}$, então

$$
\sum_{l, j=1}^{n} \bar{c}_{l} c_{j} K\left(x_{l}, x_{j}\right)=\sum_{l, j=1}^{n}\left(a_{l} a_{j}+b_{l} b_{j}\right) K\left(x_{l}, x_{j}\right)+i \sum_{l, j=1}^{n}\left(a_{l} b_{j}-a_{j} b_{l}\right) K\left(x_{l}, x_{j}\right) .
$$

Exemplo 2.1.6. Sejam $\left\{\phi_{n}\right\}$ uma seqüência de funções com domínio $X$ e $\left\{\mu_{n}\right\}$ uma seqüência de termos não-negativos. Se a série $\sum_{n=1}^{\infty} \mu_{n} \phi_{n} \otimes \overline{\phi_{n}}(x, y)$ é pontualmente convergente em $X \times X$, então, pelas propriedades acima, vemos que

$$
K(x, y):=\sum_{n=1}^{\infty} \mu_{n} \phi_{n}(x) \overline{\phi_{n}(y)}
$$

pertence a $P D(X)$. Dentro de certas condições, o Teorema 1.6.6 fornece uma espécie de recíproca deste resultado.

Vimos no Exemplo 2.1.2 que se $X$ é um conjunto não-vazio, então $P D(X) \neq \emptyset$. Se $Y \subset X$ e $K: X \times X \rightarrow \mathbb{C}$ é tal que $\left.K\right|_{Y \times Y} \in P D(Y)$, então não temos necessariamente que $K \in P D(X)$. Isto pode ser ratificado no exemplo dado abaixo e na Observação 2.2.10. Se $Y \subset X$ e $K \in P D(Y)$, a extensão $\dot{K}$ de $K$ dada por

$$
\dot{K}(x, y)=\chi_{Y}(x) K(x, y) \chi_{Y}(y), \quad x, y \in X,
$$

onde $\chi_{Y}$ é a função característica de $Y$, define um elemento de $P D(X)$. A existência de uma extensão contínua é uma questão mais complicada para a qual possivelmente não exista uma resposta geral. No artigo [39] há uma discussão sobre o assunto, quando $X \subset \mathbb{R}$.

Exemplo 2.1.7. Se $K(x, y)=(1-|x-y|), x, y \in \mathbb{R}$, então $K \chi_{[-1,1]^{2}}$ é um elemento de $P D(\mathbb{R})([39])$. Logo, se $X \subset \mathbb{R}^{m}, \max \left\{\left|x_{j}\right|: j=1,2, \ldots, m\right\} \leq 1, x \in X$, e

$$
K_{m}(x, y):=m-\sum_{1}^{m}\left|x_{i}-y_{i}\right|, \quad x, y \in X
$$

então $K_{m} \in P D(X)$. De fato, se $\left\{y^{1}, y^{2}, \ldots, y^{p}\right\} \subset X$ e $\left\{c_{1}, c_{2}, \ldots, c_{p}\right\} \subset \mathbb{R}$, então

$$
\sum_{j, l=1}^{p} c_{j} c_{l} K_{m}\left(y^{j}, y^{l}\right)=\sum_{i=1}^{m}\left(\sum_{j, l=1}^{p} c_{j} c_{l} K\left(y_{i}^{j}, y_{i}^{l}\right)\right)
$$


Por outro lado, tomando $x_{1}, x_{2} \in \mathbb{R}$, com $\left|x_{1}-x_{2}\right|=2+\epsilon, \epsilon>0$, e $0<c_{1}=c_{2} \in \mathbb{R}$, vemos que

$$
\sum_{i, j=1}^{2} c_{i} c_{j} K\left(x_{i}, x_{j}\right)=\left(\sum_{j=1}^{2} c_{j}\right)^{2}-\sum_{i, j=1}^{2} c_{i} c_{j}\left|x_{i}-x_{j}\right|=4 c_{1}^{2}-2 c_{1}^{2}(2+\epsilon)<0 .
$$

Segue que $K \notin P D(X)$ quando $X \supset[-1-\epsilon, 1+\epsilon]$. Em particular, $K_{m} \notin P D(X)$, quando $X \subset \mathbb{R}^{m}$ e $X$ contém $\left\{x \in \mathbb{R}^{m}: \max \left\{\left|x_{j}\right|: j=1,2, \ldots, m\right\} \leq 1+\epsilon\right\}$.

Para mais exemplos veja [2, p.79], [38] e [39].

Para finalizarmos a seção, vejamos mais três propriedades básicas dos núcleos positivos definidos.

Teorema 2.1.8. Se $K \in P D(X)$, então:

(i) $K(x, x) \geq 0, x \in X$;

(ii) $K$ é hermitiano;

(iii) $|K(x, y)|^{2} \leq K(x, x) K(y, y), x, y \in X$.

Demonstração: O item $(i)$ segue da definição usando-se $n=1$. O item $(i i)$ segue da definição usando-se $n=2$ e do fato de uma matriz não-negativa definida ser hermiteana. Quanto a (iii), ele segue da definição com $n=2$ e do item anterior.

Corolário 2.1.9. Seja $(X, \mathcal{M}, \mu)$ um espaço de medida $\sigma$-finito. Se $K \in P D(X)$ e a função $x \in X \mapsto K(x, x)$ pertence a $L^{1}(X)$, então $K \in L^{2}(X \times X)$ e

$$
\|K\|_{L^{2}(X \times X)} \leq \int_{X} K(x, x) d \mu(x) .
$$

Demonstração: Se $K \in P D(X)$, o Teorema 2.1.8-(iii) implica que $|K(x, y)|^{2} \leq$ $K(x, x) K(y, y), x, y \in X$. Se $x \in X \mapsto K(x, x)$ pertence a $L^{1}(X)$, podemos integrar esta desigualdade para obter

$$
\begin{aligned}
\int_{X} \int_{X}|K(x, y)|^{2} d \mu(x) d \mu(y) & \leq \int_{X} \int_{X} K(x, x) K(y, y) d \mu(x) d \mu(y) \\
& =\left(\int_{X} K(x, x) d \mu(x)\right)^{2} .
\end{aligned}
$$

Lembrando que $K(x, x) \geq 0$, a desigualdade do corolário segue.

Observação 2.1.10. No caso em que $X=\mathbb{R}^{m}$ e o núcleo $K$ do Teorema 2.1 .8 satisfaz $K(x, y)=f(x-y), x, y \in X$, as conclusões do teorema tomam a seguinte forma:

(i) $f(0) \geq 0$

(ii) $f(x)=\overline{f(-x)}$. Se $f$ for uma função real, então $f$ é par;

(iii) $|f(x)| \leq f(0), x \in \mathbb{R}^{m}$ e, em particular, $f$ é limitada. 
O exemplo seguinte satisfaz às condições da observação anterior e tem aplicações importantes em problemas de interpolação na esfera $S^{1}([18])$.

Exemplo 2.1.11. O núcleo $K(x, y):=f(x-y), x, y \in X \subset \mathbb{R}$, é positivo definido, onde

$$
f(x)=\sum_{n=0}^{\infty} \rho^{n} \cos (n x)=\frac{1-\rho \cos (x)}{1+\rho^{2}-2 \rho \cos (x)}, \quad \rho \in(0,1), \quad x \in X .
$$

\subsection{Núcleos $L^{2}$-positivos definidos}

O conceito de positividade definida tem muitas aplicações em diversas áreas da Matemática, cada contexto exigindo uma adequação conveniente do mesmo. Nesta seção introduzimos uma versão que é mais adequada ao contexto de espaços de Hilbert, não sendo, portanto, equivalente ao conceito introduzido na seção anterior.

Definição 2.2.1. Sejam $(X, \mathcal{M}, \mu)$ um espaço de medida e $K \in L^{2}(X \times X)$. Dizemos que $K$ é um núcleo $L^{2}$-positivo definido, e escrevemos $K \in L^{2} P D(X)$, quando

$$
\langle\mathcal{K}(\phi), \phi\rangle=\int_{X}\left(\int_{X} K(x, y) \phi(y) d \mu(y)\right) \overline{\phi(x)} d \mu(x) \geq 0, \quad \phi \in L^{2}(X) .
$$

Vejamos um exemplo de núcleo $L^{2}$-positivo definido.

Exemplo 2.2.2. Sejam $(X, \mathcal{M}, \mu)$ um espaço de medida e $f \in L^{2}(X)$. Como

$$
\int_{X}\left(\int_{X} f(x) \overline{f(y)} \phi(y) d \mu(y)\right) \overline{\phi(x)} d \mu(x)=\left|\int_{X} f(x) \overline{\phi(x)} d \mu(x)\right|^{2}, \quad \phi \in L^{2}(X),
$$

temos que $K:=f \otimes \bar{f} \in L^{2} P D(X)$.

A proposição abaixo indica um contexto onde as definições de positividade definida coincidem. O lema abaixo é utilizado em sua demonstração.

Lema 2.2.3. Sejam $X$ um subconjunto mensurável de $\mathbb{R}^{m}$ e $K \in L^{2}(X \times X)$. Então, $K \in L^{2} P D(X)$ se, e somente se, $\dot{K} \in L^{2} P D\left(\mathbb{R}^{m}\right)$.

Demonstração: Basta observar dois fatos: se $\phi \in L^{2}\left(\mathbb{R}^{m}\right)$, então $\left.\phi\right|_{X} \in L^{2}(X)$; se $\psi \in L^{2}(X)$ e definirmos $\dot{\psi}(x):=\chi_{X}(x) \psi(x), x \in \mathbb{R}^{m}$, então $\dot{\psi} \in L^{2}\left(\mathbb{R}^{m}\right)$.

Proposição 2.2.4. Seja $X$ um subconjunto mensurável de $\mathbb{R}^{m}$. Se $K \in P D(X), \dot{K}$ é um elemento de $L^{2}(X \times X)$ e a função

$$
(x, y) \in \mathbb{R}^{m} \times \mathbb{R}^{m} \mapsto \dot{K}(x, y) \overline{\phi(x)} \phi(y), \quad \phi \in L^{2}\left(\mathbb{R}^{m}\right) \cap C\left(\mathbb{R}^{m}\right),
$$

é Riemann-integrável, então $K \in L^{2} P D(X)$. 
Demonstração: Seja $K \in P D(X)$ e assuma que $(x, y) \in \mathbb{R}^{m} \times \mathbb{R}^{m} \mapsto \dot{K}(x, y) \overline{\phi(x)} \phi(y)$ é Riemann-integrável, para toda função $\phi \in L^{2}\left(\mathbb{R}^{m}\right) \cap C\left(\mathbb{R}^{m}\right)$. Vamos ratificar a desigualdade da Definição 2.2.1 em duas etapas. Fixado $r>0$, concluímos, utilizando a hipótese que o núcleo

$$
(x, y) \in[-r, r]^{m} \times[-r, r]^{m} \mapsto \dot{K}(x, y) \phi(y) \overline{\phi(x)}
$$

é Riemann-integrável. Particionando cada intervalo $[-r, r]$ em $n$ pontos igualmente espaçados, geramos uma malha $M_{n}$ de $[-r, r]^{m}$ formada por $n^{m}$ pontos da forma $x_{i}=$ $\left(x_{i 1}, x_{i 2} \ldots, x_{i m}\right)$, com

$$
x_{i j}=-r+(j-1) \frac{2 r}{n-1}, \quad j=1,2, \ldots, n, \quad i=1,2, \ldots, m
$$

e obtemos

$$
\int_{[-r, r]^{m}} \int_{[-r, r]^{m}} \dot{K}(x, y) \phi(y) \overline{\phi(x)} d x d y=\lim _{n \rightarrow \infty}\left(\frac{2 r}{n-1}\right)^{2 m} \sum_{l, p=1}^{n^{m}} \dot{K}\left(x_{l}, x_{p}\right) \phi\left(x_{p}\right) \overline{\phi\left(x_{l}\right)} .
$$

Lembrando o lema anterior, concluímos que

$$
\int_{[-r, r]^{m}} \int_{[-r, r]^{m}} \dot{K}(x, y) \phi(y) \overline{\phi(x)} d x d y \geq 0 .
$$

Tomando o limite quando $r \rightarrow \infty$, obtemos

$$
\int_{\mathbb{R}^{m}} \int_{\mathbb{R}^{m}} \dot{K}(x, y) \phi(y) \overline{\phi(x)} d x d y \geq 0 .
$$

Se $\phi \in L^{2}\left(\mathbb{R}^{m}\right)$, lembrando o Teorema 1.3.2, podemos encontrar uma seqüência $\left\{\phi_{n}\right\}$ de funções contínuas com suporte compacto em $\mathbb{R}^{m}$ tal que $\lim _{n \rightarrow \infty}\left\|\phi_{n}-\phi\right\|_{L^{2}\left(\mathbb{R}^{m} \times \mathbb{R}^{m}\right)}=0$. Devido ao Lema 1.6.7,

$$
\lim _{n \rightarrow \infty}\left\|\phi_{n} \otimes \overline{\phi_{n}}-\phi \otimes \bar{\phi}\right\|_{L^{2}}=0
$$

e pelo Teorema 1.4.2 segue que

$$
\int_{\mathbb{R}^{m}} \int_{\mathbb{R}^{m}} \dot{K}(x, y) \phi(y) \overline{\phi(x)} d x d y=\lim _{n \rightarrow \infty} \int_{\mathbb{R}^{m}} \int_{\mathbb{R}^{m}} \dot{K}(x, y) \phi_{n}(y) \overline{\phi_{n}(x)} d x d y \geq 0 .
$$

Utilizando o Teorema de Fubini, concluímos que $\dot{K} \in L^{2} P D\left(\mathbb{R}^{m}\right)$. Assim, pelo lema anterior, completamos a prova.

No que segue, $\partial X$ indica a fronteira do conjunto $X, X^{o}$ indica o interior do conjunto $X, C(X)$ indica o conjunto das funções contínuas de $X$ em $\mathbb{C}$, enquanto que $\operatorname{supp}(K)$ é o suporte do núcleo $K$. O próximo corolário é apenas uma aplicação da proposição que acabamos de provar. 
Corolário 2.2.5. Sejam $X$ um subconjunto mensurável de $\mathbb{R}^{m}$ e $K \in C(X \times X)$. Se $K \in P D(X) \cap L^{2}(X \times X)$ e $\partial(X)$ tem medida nula, então $K \in L^{2} P D(X)$. A mesma conclusão vale se trocarmos a condição sobre $\partial(X)$ pela seguinte: $\operatorname{supp}(K) \subset X^{o} \times X^{o}$.

Demonstração: Basta notar que o conjunto dos pontos de descontinuidade da função

$$
(x, y) \in \mathbb{R}^{m} \times \mathbb{R}^{m} \mapsto \dot{K}(x, y) \overline{\phi(x)} \phi(y), \quad \phi \in L^{2}\left(\mathbb{R}^{m}\right) \cap C\left(\mathbb{R}^{m}\right),
$$

é um conjunto de medida nula. Portanto, $\dot{K} \bar{\phi} \otimes \phi$ que pertence a $L^{1}\left(\mathbb{R}^{m} \times \mathbb{R}^{m}\right)$ é Riemann-integrável ([25, p.369]) e o resultado segue.

Vejamos alguns exemplos de núcleos que são $L^{2}$-positivos definidos e positivos definidos.

Exemplo 2.2.6. Se $X \subset \mathbb{R}^{m}$ é mensurável e tem medida finita, então os exemplos 2.2 .2 e 2.1.2 mostram que se $K(x, y):=\cos (|x|-|y|), x, y \in X$, então $K \in L^{2} P D(X) \cap$ $P D(X)$.

Exemplo 2.2.7. Se $X$ é um subconjunto mensurável de $\mathbb{R}^{m}$ e

$$
K(x, y):=\exp \left(-|x|^{2}-|y|^{2}\right), \quad x, y \in X
$$

então $K \in L^{2} P D(X) \cap P D(X)$.

O próximo resultado descreve uma situação onde a restrição de um núcleo $L^{2}$ positivo definido torna-se um núcleo positivo definido.

Definição 2.2.8. Dizemos que um subconjunto $D$ de $\mathbb{R}^{m}$ é $\partial$-mensurável quando: $D$ é mensurável, $\partial(D)=\partial\left(D^{o}\right)$ e $D^{o}$ é não-vazio.

Proposição 2.2.9. Se $D$ é $\partial$-mensurável, então $L^{2} P D(D) \cap C(D \times D) \subset P D(D)$.

Demonstração: Assuma que $D$ é $\partial$-mensurável. Sejam $K \in L^{2} P D(D) \cap C(D \times D)$, $\left\{x_{1}, x_{2}, \ldots, x_{n}\right\} \subset D^{o}$ e $\left\{c_{1}, c_{2}, \ldots, c_{n}\right\} \subset \mathbb{C}$. Tome $\epsilon>0$ de modo que o cubo $C\left[x_{j}, \epsilon\right]$ esteja em $D^{o}, j=1,2, \ldots, n$, e defina

$$
\phi_{j}(x)=\frac{1}{\left|C\left[x_{j}, \epsilon\right]\right|} \chi_{C\left[x_{i}, \epsilon\right]}(x), \quad j=1,2, \ldots, n .
$$

Como $\phi_{j} \in L^{2}(D), j=1,2, \ldots, n$, segue que $\phi(x)=\sum_{j=1}^{n} c_{j} \phi_{j}(x) \in L^{2}(D)$. Então

$$
\begin{aligned}
0 \leq \int_{D} \int_{D} K(x, y) \phi(y) \overline{\phi(x)} d x d y & =\int_{D} \int_{D} K(x, y) \sum_{i, j=1}^{n} c_{j} \phi_{j}(y) \overline{c_{i} \phi_{i}(x)} d x d y \\
& =\sum_{i, j=1}^{n} \overline{c_{i}} c_{j} \frac{1}{\left|C\left[\left(x_{i}, x_{j}\right), \epsilon\right]\right|} \int_{C\left[\left(x_{i}, x_{j}\right), \epsilon\right]} K(x, y) d x d y
\end{aligned}
$$


Usando o Teorema da Diferenciação de Lebesgue, obtemos

$$
\sum_{i, j=1}^{n} \overline{c_{i}} c_{j} K\left(x_{i}, x_{j}\right) \geq 0 .
$$

O caso geral segue aproximando-se elementos de $D$ por elementos de $D^{o}$ e usando-se argumentos de continuidade.

Observação 2.2.10. A condição $\partial(D)=\partial\left(D^{o}\right)$ na proposição anterior é essencial. De fato, seja $u=(1,1, \ldots, 1) \in \mathbb{R}^{m}$ e $Y=[-1,1]^{m} \cup\{t u: t \geq 0\}$. Sejam $f: \mathbb{R}^{m} \rightarrow \mathbb{C}$ uma função contínua tal que $f(u)=1$ e $g: \mathbb{R}^{m} \rightarrow \mathbb{C}$ uma função contínua tal que $g(u)=1$ e $g(2 u)=-1$. Se definirmos $K: Y \times Y \rightarrow \mathbb{C}$ por

$$
K(x, y)= \begin{cases}f(x) \overline{f(y)}, & x \in[-1,1]^{m} \\ g(x), & x \notin[-1,1]^{m}\end{cases}
$$

então $K \in L^{2} P D(Y) \cap C(Y \times Y) \backslash P D(Y)$. Na realidade a condição $\partial(X)=\partial\left(X^{o}\right)$ implica que $X$ não possui subconjuntos da forma $B(x, r) \cap X, x \in X$ e $r>0$, com medida nula.

Observação 2.2.11. A demonstração da proposição anterior ainda vale mesmo quando a integral

$$
\int_{D} \int_{D} K(x, y) \phi(y) \overline{\phi(x)} d x d y
$$

assume o valor $+\infty$ para algumas escolhas de $\phi \in L^{2}(D)$, ou seja, quando esta existe e é não-negativa e $K \notin L^{2}(D \times D)$.

O teorema abaixo descreve um contexto onde as duas definições de positividade definida são equivalentes.

Teorema 2.2.12. Sejam $D$ um conjunto d-mensurável e $K \in L^{2}(D \times D) \cap C(D \times D)$. Se $\partial(D)$ tem medida nula, as seguintes afirmações são equivalentes:

(i) $K \in P D(D)$;

(ii) $K \in L^{2} P D(D)$;

(iii) $\mathcal{K}$ é um operador positivo.

Demonstração: A equivalência entre $(i)$ e $(i i)$ segue do Corolário 2.2.5 e da Proposição 2.2.9. A equivalência entre $(i i)$ e (iii) é imediata.

Embora o teorema acima descreva condições sob as quais os conceitos de positividade definida são equivalentes, a Proposição 2.2.9 será mais utilizada no trabalho. O próximo resultado é um indicativo deste fato. 
Teorema 2.2.13. Seja $(X, \mathcal{M}, \mu)$ um espaço de medida $\sigma$-finito tal que $L^{2}(X)$ é separável. Se $K \in L^{2} P D(X)$, então $\mathcal{K}$ é auto-adjunto, do tipo Hilbert-Schmidt e compacto. Em particular, as conclusões do Teorema 1.5.2 valem para $\mathcal{K}$.

Demonstração: Como $\mathcal{K} \geq 0$ e o corpo envolvido é $\mathbb{C}$, pelo Teorema 1.4 .17 segue que $\mathcal{K}$ é auto-adjunto. O restante segue do Teorema 1.6.2 e do Teorema 1.6.3.

O corolário seguinte serve de motivação para a próxima seção.

Corolário 2.2.14. Sob as condições do Teorema 2.2.13 o núcleo $K$ admite uma expansão $L^{2}(X \times X)$-convergente na forma

$$
K=\sum_{n=1}^{\infty} \lambda_{n}(\mathcal{K}) \phi_{n} \otimes \overline{\phi_{n}}
$$

onde o conjunto $\left\{\phi_{n}\right\}$ é $L^{2}(X)$-ortonormal e cada $\phi_{n}$ é uma autofunção do operador $\mathcal{K}$ associada ao respectivo autovalor não-negativo $\lambda_{n}(\mathcal{K})$.

Demonstração: A demonstração deste corolário segue do Teorema 1.6.6.

\subsection{Representação de núcleos $L^{2}$-positivos definidos}

Nesta seção, assumindo-se que $D$ é um subconjunto $\partial$-mensurável e fechado de $\mathbb{R}^{m}$ bem como outras hipóteses, caracterizamos os núcleos de $L^{2} P D(D) \cap C(D \times D)$ por séries que são $L^{2}(D \times D)$, absoluta e uniformemente convergentes. As ferramentas básicas são a Observação 2.2.11 e o Teorema 2.2.13. Começamos com algumas definições de classes de funções e núcleos que aparecem na seção.

Definição 2.3.1. Seja $X$ um subconjunto de $\mathbb{R}^{m}$ e $V$ um espaço vetorial normado. Se $X$ é ilimitado, indicamos por $C_{o}(X, V)$ o conjunto das funções contínuas $f: X \rightarrow V$ que se anulam no infinito, ou seja, que satisfazem $\lim _{|x| \rightarrow \infty} f(x)=0$ em $V$. Se $X$ é limitado, escrevemos $C_{o}(X, V):=C(X, V)$.

No caso em que $V=\mathbb{C}$, escrevemos $C_{o}(X, V)=C_{o}(X)$.

Se $K: X \times X \rightarrow \mathbb{C}$ é um núcleo qualquer e $x \in X$, escrevemos $K_{x}$ para denotar a função $K_{x}: X \rightarrow \mathbb{C}$ dada por $K_{x}(y):=K(x, y), y \in X$. Similarmente, para $y \in X$ fixo, definimos a função $K^{y}: X \rightarrow \mathbb{C}$ dada por $K^{y}(x):=K(x, y), x \in X$.

Definição 2.3.2. Seja $X$ um subconjunto mensurável de $\mathbb{R}^{m}$. Dizemos que um núcleo $K: X \times X \rightarrow \mathbb{C}$ é suave quando:

(i) $K_{x} \in L^{2}(X), x \in X, e K^{y} \in L^{2}(X), y \in X$;

(ii) As funções $x \in X \mapsto K_{x} \in L^{2}(X)$ e $x \in X \mapsto K^{y} \in L^{2}(X)$ são elementos de 
$C_{o}\left(X, L^{2}(X)\right)$

(iii) $K \in C_{o}(X \times X)$.

Observação 2.3.3. Nas condições da definição, a função $x \in X \mapsto\left\|K_{x}\right\|_{L^{2}(X)}$ é um elemento de $C_{o}(X)$. De fato, a continuidade da função segue da desigualdade triangular e da continuidade de $x \in X \mapsto K_{x} \in L^{2}(X)$. Se $X$ é ilimitado, a igualdade $\lim _{|x| \rightarrow \infty}\left\|K_{x}\right\|_{L^{2}(X)}=0$ segue do item (ii) da definição e da continuidade da norma.

O próximo teorema é o principal resultado da seção. É uma versão multidimensional de resultado análogo provado em [28]. Antes do teorema, enunciamos e provamos as etapas técnicas necessárias na prova do mesmo.

Lema 2.3.4. Seja $K: X \times X \rightarrow \mathbb{C}$ um núcleo suave. Então a imagem do operador integral associado $\mathcal{K}$ é um subconjunto de $C_{o}(X)$.

Demonstração: Isto segue da igualdade

$$
\mathcal{K}(\phi)(x)=\int_{X} K(x, y) \phi(y) d y=\int_{X} \phi(y) K_{x}(y) d y=H_{\phi} \circ \overline{K_{x}}, \quad \phi \in L^{2}(X),
$$

onde $H_{\phi}(\psi)=\langle\phi, \psi\rangle, \psi \in L^{2}(X)$, e do item (ii) da definição.

Lema 2.3.5. Seja $D$ um subconjunto d-mensurável de $\mathbb{R}^{m}$. Seja $K: D \times D \rightarrow \mathbb{C}$ um núcleo suave cujo operador integral associado $\mathcal{K}$ possui uma representação espectral $L^{2}(D)$-convergente na forma

$$
\mathcal{K}(f)=\sum_{n=1}^{\infty} \lambda_{n}(\mathcal{K})\left\langle f, \phi_{n}\right\rangle \phi_{n}, \quad f \in L^{2}(D),
$$

onde $\left\{\phi_{n}\right\}$ é um subconjunto ortonormal de $L^{2}(D)$ e $\left\{\lambda_{n}(\mathcal{K})\right\} \subset[0, \infty)$ é a seqüência de autovalores de $\mathcal{K}$. Então a série

$$
\sum_{n=1}^{\infty} \lambda_{n}(\mathcal{K})\left|\phi_{n}(x)\right|^{2}, \quad x \in D,
$$

converge absolutamente em D. Em particular

$$
0 \leq \sum_{n=1}^{\infty} \lambda_{n}(\mathcal{K})\left|\phi_{n}(x)\right|^{2} \leq K(x, x), \quad x \in D .
$$

Demonstração: Podemos assumir, sem perda de generalidade, que $\lambda_{n}(\mathcal{K})>0, n=$ $1,2, \ldots$ Fixemos $p \geq 1$ e consideremos o núcleo $K_{p}$ dado por

$$
K_{p}(x, y)=K(x, y)-\sum_{n=1}^{p} \lambda_{n}(\mathcal{K}) \phi_{n}(x) \overline{\phi_{n}(y)}, \quad x, y \in D
$$


O fato de $K$ ser suave e o Lema 2.3.4 mostram que $K_{p} \in C_{o}(D \times D)$. Se $\mathcal{K}_{p}$ é o operador integral com núcleo $K_{p}$, podemos utilizar o Teorema de Fubini e o Teorema 1.4.2 para obter

$$
\begin{aligned}
\left\langle\mathcal{K}_{p}(f), f\right\rangle & =\int_{D}\left[\int_{D}\left(K(x, y)-\sum_{1}^{p} \lambda_{n}(\mathcal{K}) \phi_{n}(x) \overline{\phi_{n}(y)}\right) f(y) d y\right] \overline{f(x)} d x \\
= & \int_{D}\left(\sum_{n=1}^{\infty} \lambda_{n}(\mathcal{K})\left\langle f, \phi_{n}\right\rangle \phi_{n}(x)\right) \overline{f(x)} d x \\
& \quad-\sum_{n=1}^{p} \int_{D} \int_{D} \lambda_{n}(\mathcal{K}) \phi_{n}(x) \overline{\phi_{n}(y)} f(y) \overline{f(x)} d y d x \\
= & \sum_{n=p+1}^{\infty} \lambda_{n}(\mathcal{K})\left|\left\langle f, \phi_{n}\right\rangle\right|^{2}, \quad f \in L^{2}(D) .
\end{aligned}
$$

Em particular, $\left\langle\mathcal{K}_{p}(f), f\right\rangle \geq 0, f \in L^{2}(D)$, ou seja, $\mathcal{K}_{p}$ é um operador positivo. Pela Observação 2.2.11, $K_{p}(x, x) \geq 0, x \in D$. Logo, como $p$ é arbitrário, segue que

$$
0 \leq \sum_{n=1}^{\infty} \lambda_{n}(\mathcal{K})\left|\phi_{n}(x)\right|^{2} \leq K(x, x)<\infty, \quad x \in D .
$$

A prova está completa.

Lema 2.3.6. Assuma que as hipóteses do Lema 2.3.5 valem e que D é fechado. Fixado $x \in D$, a série

$$
\sum_{n=1}^{\infty} \lambda_{n}(\mathcal{K}) \phi_{n}(x) \overline{\phi_{n}(y)}
$$

converge absoluta e uniformemente em $D$ para a função contínua $B_{x}$ dada por $B_{x}(y):=$ $B(x, y), y \in D$. A mesma conclusão é válida trocando-se a ordem das variáveis.

Demonstração: A suavidade de $K$ garante que $M=\max _{y \in D} K(y, y)<\infty$. Utilizando a desigualdade de Cauchy-Schwarz e o Lema 2.3.5 deduzimos que para $1 \leq p \leq q$,

$$
\left|\sum_{n=p}^{q} \lambda_{n}(\mathcal{K}) \phi_{n}(x) \overline{\phi_{n}(y)}\right|^{2} \leq M \sum_{n=p}^{q} \lambda_{n}(\mathcal{K})\left|\phi_{n}(x)\right|^{2}, \quad x, y \in D,
$$

conseqüentemente, pelo critério de Cauchy ([35, p.147]), a convergência da série $\sum_{n=1}^{\infty} \lambda_{n}(\mathcal{K})\left|\phi_{n}(x)\right|^{2}$ implica na convergência da série

$$
\sum_{n=1}^{\infty} \lambda_{n}(\mathcal{K}) \phi_{n}(x) \overline{\phi_{n}(y)}
$$


em $D \times D$, absolutamente em $x$ e uniformemente em $y$. Sendo assim, fixado $x \in D$, sua soma $B_{x}(y)=B(x, y)$ é contínua em relação a $y$, pelo Teorema 1.2.4. De maneira análoga, concluímos que, fixado $y \in D, B(x, y)$ é contínua em relação a $x$.

Note que o lema anterior continua válido se trocarmos a hipótese "D fechado" por " $K$ é limitado".

Teorema 2.3.7. Seja $D$ um subconjunto d-mensurável e fechado de $\mathbb{R}^{m}$. Seja $K: D \times$ $D \rightarrow \mathbb{C}$ um núcleo suave cujo operador integral associado $\mathcal{K}$ possui uma representação espectral $L^{2}(D)$-convergente na forma

$$
\mathcal{K}(f)=\sum_{n=1}^{\infty} \lambda_{n}(\mathcal{K})\left\langle f, \phi_{n}\right\rangle \phi_{n}, \quad f \in L^{2}(D),
$$

onde $\left\{\phi_{n}\right\}$ é um subconjunto ortonormal de $L^{2}(D)$ e a seqüência $\left\{\lambda_{n}(\mathcal{K})\right\}$ de autovalores de $\mathcal{K}$ é um subconjunto de um setor circular de ângulo $<\pi$ e vértice na origem de $\mathbb{C}$. Então a série

$$
\sum_{n=1}^{\infty} \lambda_{n}(\mathcal{K}) \phi_{n}(x) \overline{\phi_{n}(y)}
$$

converge absoluta e uniformemente para $K$ em $D \times D$. Ainda, se $\lambda_{n}(\mathcal{K})$ é não-nulo, então $\phi_{n} \in C_{o}(D)$.

Demonstração: Vamos demonstrar o teorema usando um operador integral auxiliar. Para tanto, escolhamos $\alpha \in[0,2 \pi]$ de modo que os autovalores

$$
\alpha_{n}=e^{i \alpha} \lambda_{n}(\mathcal{K}):=\alpha_{n}^{1}+i \alpha_{n}^{2}, \quad n=1,2, \ldots,
$$

do operador $P:=e^{i \alpha} \mathcal{K}$ estejam em um setor circular limitado pelos raios $t= \pm l s$, $s \geq 0$, para algum $l>0$. O operador $T:=\left(P+P^{*}\right) / 2$ tem, pelo Corolário 1.4.9, forma espectral

$$
T(f)=\sum_{n=1}^{\infty} \alpha_{n}^{1}(T)\left\langle f, \phi_{n}\right\rangle \phi_{n}, \quad f \in L^{2}(D),
$$

com $\alpha_{n}^{1}(T)=\alpha_{n}^{1} \geq 0$. Ele é um operador integral com núcleo

$$
L(x, y)=\frac{e^{i \alpha} K(x, y)+e^{-i \alpha} \overline{K(y, x)}}{2}
$$

que é suave. Pelo Lema 2.3.6 concluímos que, para cada $x \in D$ fixo, a série

$$
\sum_{n=1}^{\infty} \alpha_{n}^{1} \phi_{n}(x) \overline{\phi_{n}(y)}, \quad y \in D
$$


converge absoluta e uniformemente para uma função contínua $B_{x}(y)=B(x, y), y \in D$. O próximo passo da prova consiste em concluir que $B(x, y)=L(x, y), x, y \in D$. Feito isso a convergência uniforme da série

$$
\sum_{n=1}^{\infty} \alpha_{n}^{1} \phi_{n}(x) \overline{\phi_{n}(y)}, \quad x, y \in D
$$

seguirá. Aplicando a desigualdade de Bessel e a Observação 2.3.3 obtemos

$$
\sum_{n=1}^{\infty}\left(\alpha_{n}^{1}\right)^{2}\left|\phi_{n}(x)\right|^{2}=\sum_{n=1}^{\infty}\left|\left\langle\phi_{n}, \overline{L_{x}}\right\rangle\right|^{2} \leq\left\|L_{x}\right\|_{L^{2}(D)}^{2} \leq \max _{x \in D}\left\{\left\|L_{x}\right\|_{L^{2}(D)}^{2}\right\}<\infty .
$$

Logo, pelo Teorema de Riesz-Fisher, concluímos que

$$
\sum_{n=1}^{\infty}\left(\alpha_{n}^{1} \phi_{n}(x)\right) \overline{\phi_{n}} \in L^{2}(D), \quad x \in D
$$

ou seja, $B_{x} \in L^{2}(D), x \in D$. Além disso, pelo Teorema 1.4.2, vale

$$
\int_{D} B(x, y) f(y) d y=\sum_{n=1}^{\infty} \alpha_{n}^{1}\left\langle f, \phi_{n}\right\rangle \phi_{n}(x), \quad f \in L^{2}(D), \quad x \in D .
$$

A série acima converge uniformemente, pois, pelas desigualdades de Cauchy-Schwarz e Bessel e as estimativas acima,

$$
\begin{aligned}
\left|\sum_{n=p}^{q} \alpha_{n}^{1} \phi_{n}(x)\left\langle f, \phi_{n}\right\rangle\right|^{2} & \leq \sum_{n=p}^{q}\left(\alpha_{n}^{1}\right)^{2}\left|\phi_{n}(x)\right|^{2} \sum_{n=p}^{q}\left|\left\langle f, \phi_{n}\right\rangle\right|^{2} \\
& \leq \max _{x \in D}\left\{\left\|L_{x}\right\|_{L^{2}(D)}^{2}\right\} \sum_{n=p}^{q}\left|\left\langle f, \phi_{n}\right\rangle\right|^{2} .
\end{aligned}
$$

Voltando à definição de $T$, notamos agora que

$$
\int_{D}[L(x, y)-B(x, y)] f(y) d y=0, \quad x \in D, \quad f \in L^{2}(D) .
$$

Fixado $x \in D$, as funções $B(x, \cdot)$ e $L(x, \cdot)$ coincidem quase sempre. Como $D$ não possui pontos isolados, tem medida positiva e as funções acima são contínuas em $y$, temos que $B(x, \cdot)=L(x, \cdot), x \in D$. Em particular,

$$
B(x, x)=L(x, x)=\sum_{n=1}^{\infty} \alpha_{n}^{1}\left|\phi_{n}(x)\right|^{2}, \quad x \in D .
$$

Sendo assim, para cada $x \in D$, a seqüência $\left\{B_{p}(x, x)\right\}=\left\{\sum_{n=1}^{p} x_{n}\left|\phi_{n}(x)\right|^{2}\right\}$ é monótona e pontualmente convergente para $L(x, x)$. Por outro lado, o Lema 2.3.4 implica que 
as autofunções $\phi_{n}$ de $T$ associadas aos autovalores não nulos são elementos de $C_{o}(D)$. Logo, as funções $x \in D \mapsto B_{p}(x, x)$ e $x \in D \mapsto L(x, x)$ são elementos de $C_{o}(D)$. Portanto, pelo Teorema 1.2.3 e pelo Teorema de Dini, a série da igualdade acima converge uniformemente em $D$. Utilizando a desigualdade de Cauchy-Schwarz para escrever

$$
\left|\sum_{n=p}^{q} \alpha_{n}^{1} \phi_{n}(x) \overline{\phi_{n}(y)}\right|^{2} \leq \sum_{n=p}^{q} \alpha_{n}^{1}\left|\phi_{n}(x)\right|^{2} \sum_{n=p}^{q} \alpha_{n}^{1}\left|\phi_{n}(y)\right|^{2}, \quad q \geq p,
$$

concluímos que a série dupla (2.1) converge absoluta e uniformemente para $L$ em $D \times D$. A convergência da série do enunciado pode agora ser analisada. Usando a estimativa

$$
\left|\alpha_{n}\right|^{2}=\left|\lambda_{n}(\mathcal{K})\right|^{2}=\left(\alpha_{n}^{1}\right)^{2}+\left(\alpha_{n}^{2}\right)^{2} \leq\left(\alpha_{n}^{1}\right)^{2}\left(1+l^{2}\right), \quad n=1,2, \ldots,
$$

e a desigualdade de Cauchy-Schwarz mais uma vez, obtemos (para $q \geq p \geq 1$ )

$$
\left|\sum_{n=p}^{q} \lambda_{n}(\mathcal{K}) \phi_{n}(x) \overline{\phi_{n}(y)}\right|^{2} \leq\left(1+l^{2}\right) \sum_{n=p}^{q} \alpha_{n}^{1}\left|\phi_{n}(x)\right|^{2} \sum_{n=p}^{q} \alpha_{n}^{1}\left|\phi_{n}(y)\right|^{2} .
$$

Logo, a convergência da séria dupla é absoluta e uniforme em $D \times D$. Sua soma $G$ é necessariamente um elemento de $C_{o}(D \times D)$. Agora, repetindo o raciocínio empregado na conclusão da igualdade entre $B$ e $L$ para os núcleos $G$ e $K$, a partir da equação (2.2), concluímos que $G=K$ em $D \times D$. A prova está completa.

Terminamos esta seção com uma versão mais fraca do teorema anterior, já adaptada ao contexto do trabalho.

Teorema 2.3.8. Seja $D$ um subconjunto d-mensurável e fechado de $\mathbb{R}^{m}$. Se $K \in$ $L^{2} P D(D)$ é um núcleo suave, então valem as seguintes afirmações:

(i) $\operatorname{Im} \mathcal{K} \subset C_{o}(D) \cap L^{2}(D)$.

(ii) $K$ é representável por uma série $L^{2}(D \times D)$, absoluta e uniformemente convergente da forma

$$
K(x, y)=\sum_{n=1}^{\infty} \lambda_{n}(\mathcal{K}) \phi_{n}(x) \overline{\phi_{n}(y)}, \quad x, y \in D,
$$

sendo $\left\{\phi_{n}\right\}$ um conjunto $L^{2}(D)$-ortonormal e $\lambda_{1}(\mathcal{K}) \geq \lambda_{2}(\mathcal{K}) \geq \cdots \geq 0$.

Demonstração: Utilizando o Teorema 2.2.13 e o Corólário 1.5.3 temos que $\mathcal{K}$ é representável por uma série $L^{2}(D)$-convergente da forma

$$
\mathcal{K}(f)=\sum_{n=1}^{\infty} \lambda_{n}(\mathcal{K})\left\langle f, \phi_{n}\right\rangle \phi_{n}, \quad f \in L^{2}(D),
$$

onde $\left\{\lambda_{n}(\mathcal{K})\right\}$ são os autovalores (não-negativos) de $\mathcal{K}$ e $\left\{\phi_{n}\right\}$ é uma base $L^{2}(D)$ ortonormal de autofunções de $\mathcal{K}$. Logo, os itens $(i)$ e $(i i)$ seguem do Lema 2.3.4 e do Teorema 2.3.7. 
Observação 2.3.9. As definições desta seção são relevantes apenas quando $D$ não é compacto. De fato, se $D$ é compacto, algumas hipóteses nos resultados da seção podem ser retiradas, uma vez que as mesmas já estarão satisfeitas em função da continuidade do núcleo. Isto motiva a discussão da seção seguinte.

\subsection{Núcleos $L^{2}$-positivos definidos: generalizações}

É possível encontrar na literatura versões diferentes para o Teorema 2.3.7. Em [20], Kühn estabelece uma versão para núcleos em $L^{2} P D(X)$, onde $X$ é um espaço de Hausdorff compacto, munido de uma medida de Borel finita e não-nula $\mu$, cujo suporte é o próprio $X$ (se $Y \subset X$ é aberto e não-vazio, então $\mu(Y)>0$ ). O método empregado na prova do Teorema 2.3.7 pode ser adaptado para cobrir a seguinte situação: $X$ é um espaço topológico compacto munido de uma medida de Borel finita e não-nula $\mu$, $X$ é o suporte de $\mu$ e $L^{2}(X, \mu)$ é separável. Embora estejamos interessados apenas em subconjuntos de $\mathbb{R}^{m}$, dedicamos esta parte do trabalho a esta variante.

A menos de especificação em contrário, nesta seção, $X$ é um espaço topológico compacto munido de uma medida de Borel finita $\mu$ tal que $\mu(X)>0$. Começamos nosso estudo com os seguintes lemas.

Lema 2.4.1. Seja $(Y, \mathcal{M}, \mu)$ um espaço de medida, com $\mu(Y)<\infty$. Se K é um elemento hermitiano de $L^{2}(Y \times Y, \mu \times \mu)$, então

$$
\int_{Y} \int_{Y} K(x, y) d \mu(x) d \mu(y)=\int_{Y} \int_{Y} \operatorname{Re}(K(x, y)) d \mu(x) d \mu(y),
$$

Demonstração: Pelo Corolário 1.3.5 sabemos que $L^{2}(Y \times Y, \mu \times \mu) \subset L^{1}(Y \times Y, \mu \times \mu)$. Pelo Teorema de Fubini, $K(\cdot, y) \in L^{1}(Y, \mu)$ quase sempre e

$$
\begin{aligned}
\int_{Y} \int_{Y} K(x, y) d \mu(x) d \mu(y) & =\int_{Y}\left[\int_{Y} K(x, y) d \mu(x)\right] d \mu(y) \\
& =\int_{Y}\left[\int_{Y} K(x, y) d \mu(y)\right] d \mu(x) \\
& =\int_{Y}\left[\int_{Y} K(y, x) d \mu(x)\right] d \mu(y) .
\end{aligned}
$$

Por outro lado, pelo mesmo teorema

$$
\begin{aligned}
& \overline{\int_{Y} \int_{Y} K(x, y) d \mu(x) d \mu(y)}=\overline{\int_{Y}\left[\int_{Y} K(x, y) d \mu(x)\right] d \mu(y)} \\
& =\int_{Y} \overline{\left[\int_{Y} K(x, y) d \mu(x)\right]} d \mu(y) \\
& =\int_{Y}\left[\int_{Y} \overline{K(x, y)} d \mu(x)\right] d \mu(y) \text {. }
\end{aligned}
$$


Utilizando a hipótese temos que as duas equações acima são iguais. Portanto,

$$
\int_{Y} \int_{Y} K(x, y) d \mu(x) d \mu(y)=\overline{\int_{Y} \int_{Y} K(x, y) d \mu(x) d \mu(y)}
$$

e o resultado segue.

O lema abaixo é um corolário da demonstração do Lema 2.3.4.

Lema 2.4.2. Se $K \in C(X \times X)$ as seguintes afirmações são válidas:

(i) A função $x \in X \mapsto\left\|K_{x}\right\|_{L^{2}(X, \mu)}$ é um elemento de $C(X)$;

(ii) $\operatorname{Im} \mathcal{K} \subset C(X)$.

Demonstração: Basta notar que a continuidade de $K$ e a compacidade de $X$ implicam que $K_{x} \in L^{2}(X, \mu)$. Como $\mu(X)<\infty$, pelo Teorema da Convergência Dominada segue que $x \in X \mapsto K_{x}$ é contínua. Procedendo-se como na Observação 2.3.3 e na demonstração do Lema 2.3.4, obtemos os demais resultados.

Lema 2.4.3. Assuma que $X$ é o suporte de $\mu$. Se $K \in L^{2} P D(X) \cap C(X \times X)$, então $K$ é hermitiano e $K(x, x) \geq 0, x \in X$.

Demonstração: Nas condições do enunciado, pelo Teorema 1.4.17 o operador integral $\mathcal{K}$ é auto-adjunto. Entretanto, alguns cálculos utilizando o Teorema de Fubini e a unicidade do operador adjunto de $\mathcal{K}$, mostram que $K^{*}(x, y):=\overline{K(y, x)}$ é o núcleo de $\mathcal{K}^{*}$, ou seja,

$$
\int_{X}[K(x, y)-\overline{K(y, x)}] \phi(y) d \mu(y)=0, \quad \phi \in L^{2}(X, \mu),
$$

quase sempre. Como $K$ é contínua, utilizando o Lema 2.4.2-(ii), concluímos que a equação acima é nula para todo $x \in X$. Além disso, a função $y \in X \mapsto \phi_{x}(y):=$ $\overline{K(x, y)}-K(y, x)$ é um elemento de $L^{2}(X, \mu) \cap C(X)$, para todo $x \in X$. Logo, da equação acima

$$
\int_{X}\left|\phi_{x}(y)\right|^{2} d \mu(y)=0
$$

Suponhamos que existam $z, w \in X$ tais que $K(z, w) \neq \overline{K(w, z)}$. Da continuidade de $\phi_{z}$ segue que $Y=\left(\phi_{z}\right)^{-1}(\mathbb{C} \backslash\{0\}) \neq \emptyset$ é aberto e possui medida positiva, pois $X$ é o suporte de $\mu$. Mas se isto ocorrer teremos

$$
\int_{Y}\left|\phi_{x}(y)\right|^{2} d \mu(y)=0
$$

o que é absurdo, pelo Teorema 1.3.7. Para mostrarmos que $K(x, x) \geq 0, x \in X$, suponhamos que existe $x_{o} \in X$ tal que $K\left(x_{o}, x_{o}\right)<0$. Como $K \in C(X \times X)$, existe uma vizinhança aberta e com medida positiva e finita $V_{x_{o}}$ de $x_{o}$ tal que $\operatorname{Re}(K(x, y))<0$, 
$x, y \in V_{x_{o}}$. Tomando $f:=\chi_{V_{x_{o}}} \in L^{2}(X, \mu)$, utilizando o Lema 2.4.1 e o Teorema 1.3.7, obtemos

$$
\langle\mathcal{K}(f), f\rangle=\int_{V_{x_{o}}} \int_{V_{x_{o}}} K(x, y) d \mu(x) d \mu(y)<0,
$$

contradizendo o fato de $K \in L^{2} P D(X)$.

Note que o Lema 2.4.3 ainda é verdadeiro quando $X$ é um subconjunto nãocompacto de $\mathbb{R}^{m}$ com a topologia usual e $K$ é um núcleo suave, desde que $X$ seja o suporte da restrição da medida de Lebesgue.

Lema 2.4.4. Assuma que $X$ é o suporte de $\mu$. Se $K \in C(X \times X)$ e o operador integral $\mathcal{K}$ possui uma representação espectral $L^{2}(X, \mu)$-convergente na forma

$$
\mathcal{K}(f)=\sum_{n=1}^{\infty} \lambda_{n}(\mathcal{K})\left\langle f, \phi_{n}\right\rangle \phi_{n}, \quad f \in L^{2}(X, \mu),
$$

onde $\left\{\phi_{n}\right\}$ é um subconjunto ortonormal de $L^{2}(X, \mu)$ e $\left\{\lambda_{n}(\mathcal{K})\right\} \subset[0, \infty)$ é a seqüência de autovalores de $\mathcal{K}$, então a série

$$
\sum_{n=1}^{\infty} \lambda_{n}(\mathcal{K})\left|\phi_{n}(x)\right|^{2}, \quad x \in X,
$$

converge absolutamente em $X$. Em particular

$$
0 \leq \sum_{n=1}^{\infty} \lambda_{n}(\mathcal{K})\left|\phi_{n}(x)\right|^{2} \leq K(x, x), \quad x \in X .
$$

Demonstração: Segue da demonstração do Lema 2.3.5, trocando-se nos argumentos, o Lema 2.3.4 pelo Lema 2.4.2 e a Proposição 2.2.9 pelo Lema 2.4.3.

Lema 2.4.5. Sob as condições do Lema 2.4.4, fixado $x \in X$, a série

$$
\sum_{n=1}^{\infty} \lambda_{n}(\mathcal{K}) \phi_{n}(x) \overline{\phi_{n}(y)}
$$

converge absoluta e uniformemente em $X$ para uma função contínua $B_{x}$, onde $B_{x}(y):=$ $B(x, y)$. A mesma conclusão é válida trocando-se a ordem das variáveis.

Demonstração: Segue da demonstração do Lema 2.3.6, utilizando na argumentação o Lema 2.4.4 no lugar do Lema 2.3.5.

Teorema 2.4.6. Assuma que $X$ é o suporte de $\mu$. Se $K \in C(X \times X)$ e o operador integral associado $\mathcal{K}$ possui uma representação espectral $L^{2}(X, \mu)$-convergente na forma

$$
\mathcal{K}(f)=\sum_{n=1}^{\infty} \lambda_{n}(\mathcal{K})\left\langle f, \phi_{n}\right\rangle \phi_{n}, \quad f \in L^{2}(X, \mu),
$$


onde $\left\{\phi_{n}\right\}$ é um subconjunto ortonormal de $L^{2}(X, \mu)$ e a seqüência $\left\{\lambda_{n}(\mathcal{K})\right\}$ de autovalores de $\mathcal{K}$ é um subconjunto de um setor circular de ângulo $<\pi$ e vértice na origem de $\mathbb{C}$, então a série

$$
\sum_{n=1}^{\infty} \lambda_{n}(\mathcal{K}) \phi_{n}(x) \overline{\phi_{n}(y)}
$$

converge absoluta e uniformemente para $K$ em $X \times X$. Ainda, se $\lambda_{n}(\mathcal{K})$ é não-nulo, então $\phi_{n} \in C(X)$.

Demonstração: Segue da demonstração do Teorema 2.3.7 e dos lemas anteriores.

Teorema 2.4.7. Assuma que $X$ é o suporte de $\mu$ e que $L^{2}(X, \mu)$ é separável. Se $K \in L^{2} P D(X) \cap C(X \times X)$, então as seguintes afirmações valem:

(i) $\operatorname{Im} \mathcal{K} \subset C(X) \cap L^{2}(X, \mu)$;

(ii) $K$ é representável por uma série $L^{2}(X \times X, \mu \times \mu)$, absoluta e uniformemente convergente da forma

$$
K(x, y)=\sum_{n=1}^{\infty} \lambda_{n}(\mathcal{K}) \phi_{n}(x) \overline{\phi_{n}(y)}, \quad x, y \in X,
$$

onde $\left\{\phi_{n}\right\}$ é um conjunto $L^{2}(X, \mu)$-ortonormal formado por autofunções do operador integral $\mathcal{K}$ associadas aos autovalores não-negativos $\lambda_{n}(\mathcal{K})$.

(iii) $O$ operador $\mathcal{K}$ é nuclear e

$$
\operatorname{tr}(|\mathcal{K}|)=\operatorname{tr}(\mathcal{K})=\sum_{n=1}^{\infty} \lambda_{n}(\mathcal{K})=\int_{X} K(x, x) d \mu(x)<\infty .
$$

Demonstração: Os itens $(i)$ e (ii) seguem da demonstração do Teorema 2.3.8 e dos lemas anteriores. Para o item (iii), observe que a convergência da série (2.3) é uniforme e que a seqüência

$$
\sum_{n=1}^{k} \lambda_{n}(\mathcal{K})\left|\phi_{n}(x)\right|^{2}
$$

é não-decrescente e convergente para $K(x, x)$. O resultado segue pelo Teorema da Convergência Monótona.

Os resultados obtidos nesta seção nos permitem provar agora uma generalização da Proposição 2.2.9.

Teorema 2.4.8. Se $X$ é o suporte de $\mu$ e $L^{2}(X, \mu)$ é separável, então $L^{2} P D(X) \cap$ $C(X \times X) \subset P D(X)$.

Demonstração: Segue diretamente do teorema anterior e das propriedades dos núcleos positivos definidos. 
Observação 2.4.9. Assumindo-se que o espaço $(X, \mathcal{M}, \mu)$ é tão somente um espaço com medida finita, não podemos garantir a mensurabilidade dos conjuntos abertos e das funções contínuas, informações utilizadas em argumentos importantes das provas dos resultados anteriores. Logo, com essa hipótese não há garantia de validade dos resultados. Por outro lado, observamos que os resultados continuam válidos, quando substituimos a medida de Borel pelo seu completamento. Em particular, os resultados valem quando $X$ é um subconjunto de $\mathbb{R}^{m}$ e a medida é a restrição da medida de Lebesgue.

Os resultados abaixo capturam algumas escolhas importantes para o espaço $X$.

Corolário 2.4.10. Seja $S^{m}$ a esfera unitária de $\mathbb{R}^{m+1}, m \geq 1$, munida da medida de Lebesgue usual. Se $X$ é um subconjunto fechado de $S^{m}$, então os teoremas 2.4.6 e 2.4.7 são ainda verdadeiros, contanto que $X$ seja o suporte da restrição da medida de Lebesgue $\sigma_{m}$ a $X$.

Demonstração: Basta notar que $L^{2}\left(S^{m}, \sigma_{m}\right)$ é separável $([16$, p.71]) e utilizar a Observação 2.4.9.

O teorema abaixo contém o Teorema de Mercer e generaliza um pouco mais os resultados obtidos até o momento.

Teorema 2.4.11. Seja $X$ é um subconjunto fechado de $\mathbb{R}^{m}$ com medida positiva e que coincide com o suporte da restrição da medida de Lebesgue a $X$.

(i) Se X for limitado, então o Teorema 2.4.6 e o Teorema 2.4.7 são verdadeiros.

(ii) Se $X$ for ilimitado, então as conclusões do Teorema 2.3.7 e do Teorema 2.3.8 ainda são válidas.

Demonstração: O item $(i)$ segue da Observação 2.4.9. Para o item $(i i)$, basta proceder como na demonstração dos teoremas 2.3 .7 e 2.3.8, substituindo o Lema 2.3.6 pelo Lema 2.4.5 nos argumentos.

Os resultados obtidos nesta seção nos permitem provar agora mais uma generalização da Proposição 2.2.9.

Teorema 2.4.12. Se $Y$ é um subconjunto fechado de $\mathbb{R}^{m}$ que coincide com o suporte da restrição da medida de Lebesgue de $\mathbb{R}^{m}$ a $Y$, então $L^{2} P D(Y) \cap C(Y \times Y) \subset P D(Y)$.

Demonstração: Sejam $\left\{x_{1}, x_{2}, \ldots, x_{n}\right\}$ um subconjunto de $Y$ e $r>0$. Como $X$ é o suporte da restrição da medida de Lebesgue, então $X=\cup_{j=1}^{n}\left(B\left(x_{j}, r\right) \cap Y\right)$ também é o suporte da restrição da medida. Além disso, $Z:=\bar{X} \cap Y$, onde $\bar{X}$ denota o fecho de $X$, é um conjunto compacto, contém o conjunto $\left\{x_{1}, x_{2}, \ldots, x_{n}\right\}$ e é também o 
suporte da restrição da medida. Aplicando o Teorema 2.4.8 ao núcleo $K_{\mid Z}$ concluímos que $K_{\mid Z} \in P D(Z)$. Voltando à definição de núcleos positivos definidos concluímos a prova.

A prova da recíproca do teorema anterior não é do nosso conhecimento. Entretanto, acreditamos que seja verdadeira com base nas afirmações de Kühn [20] e Schaback [37].

\subsection{Núcleos $L^{2}$-positivos definidos: caracterização}

Nesta seção refinamos os resultados obtidos na seção anterior. Mais específicamente, assumindo-se que $D$ é um subconjunto fechado de $\mathbb{R}^{m}$ que coincide com o suporte da restrição da medida de Lebesgue de $\mathbb{R}^{m}$ a $D$, caracterizamos os núcleos $K$ contínuos e em $L^{2} P D(D)$ para os quais a função $x \in D \mapsto K(x, x)$ pertence a $L^{1}(D) \cap C_{o}(D)$. Mostramos que tais núcleos possuem uma expansão em série que é $L^{2}(D \times D)$, absoluta e uniformemente convergente, cujo operador integral associado é nuclear. No caso em que a função $x \in D \mapsto K(x, x)^{1 / 2}$ também pertence a $L^{1}(D)$, obtemos ainda a convergência no sentido $L^{1}$ e estimativas para a norma de $K$ e das autofunções associadas aos autovalores não nulos de $\mathcal{K}$.

Começamos com as seguintes definições.

Definição 2.5.1. Dizemos que um subconjunto $X$ de $\mathbb{R}^{m}$ tem medida estritamente positiva se $X$ é igual ao suporte da restrição da medida de Lebesgue de $\mathbb{R}^{m}$ a $X$.

Definição 2.5.2. Seja $X$ um subconjunto mensurável de $\mathbb{R}^{m}$. O conjunto $\mathcal{A}_{o}(X)$ é o subconjunto de $C_{o}(X \times X) \cap L^{2} P D(X)$ formado por todos os núcleos $K$ para os quais a função $x \in X \mapsto K(x, x)$ é um elemento de $L^{1}(X)$.

Note que a Proposição 2.2.9 e o Teorema 2.4.12 justificam a inclusão $\mathcal{A}_{o}(D) \subset$ $P D(D)$ no caso em que $D$ é $\partial$-mensurável ou $D$ é fechado e tem medida estritamente positiva. Note também que os conjuntos $\partial$-mensuráveis têm medida estritamente positiva.

Lema 2.5.3. Seja $D$ um subconjunto fechado de $\mathbb{R}^{m}$. Se D tem medida estritamente positiva e $K \in \mathcal{A}_{o}(D)$, então $K$ é um núcleo suave.

Demonstração: Pelo Teorema 2.1.8, já sabemos que $|K(x, y)|^{2} \leq K(x, x) K(y, y)$, $x, y \in D$. Logo,

$$
\int_{D}\left|K_{x}(y)\right|^{2} d y=\int_{D}|K(x, y)|^{2} d y \leq K(x, x) \int_{D} K(y, y) d y, \quad x \in D .
$$

Em particular, $K_{x} \in L^{2}(D), x \in D$. A igualdade $K_{x}(y)=\overline{K^{y}(x)}, x, y \in D$, revela que $K^{y} \in L^{2}(D), y \in D$. Analisemos agora a continuidade da função $x \in D \mapsto K_{x} \in L^{2}(D)$ 
em um ponto arbitrário $x_{0}$. Podemos supor que $K(x, x) \neq 0$ para algum $x \in D$, já que, caso contrário, as condições do lema podem ser trivialmente verificadas. Para tanto, vamos estimar

$$
\left\|K_{x}-K_{x_{0}}\right\|_{L^{2}(D)}^{2}=\int_{D}\left|K(x, y)-K\left(x_{0}, y\right)\right|^{2} d y
$$

convenientemente. Seja $\epsilon>0$ e defina $M:=\max _{x \in D}\{K(x, x)\}$. Obviamente, $M \in$ $(0, \infty)$. Como a função não-negativa $y \in D \mapsto K(y, y)$ é um elemento de $L^{1}(D)$, é possível escolhermos $r>0$ suficientemente grande, de tal forma que o cubo $[-r, r]^{m}$ contém $x_{0}$ em seu interior, $Q:=D \cap[-r, r]^{m}$ tem medida positiva e

$$
\int_{D \backslash Q} K(y, y) d y<\frac{\epsilon}{5 M}
$$

Analisamos agora cada integral na decomposição abaixo:

$$
\begin{aligned}
\int_{D}\left|K(x, y)-K\left(x_{0}, y\right)\right|^{2} d y=\int_{Q} \mid K(x, y) & -\left.K\left(x_{0}, y\right)\right|^{2} d y \\
& +\int_{D \backslash Q}\left|K(x, y)-K\left(x_{0}, y\right)\right|^{2} d y
\end{aligned}
$$

Sendo $K$ uniformemente contínuo em $Q \times Q$, existe uma vizinhança aberta $V_{x_{0}}$ de $x_{0}$ tal que

$$
\left|K(x, y)-K\left(x_{0}, y\right)\right|^{2}<\frac{\epsilon}{5|Q|}, \quad y \in Q, \quad x \in V_{x_{0}} \cap Q
$$

Logo,

$$
\int_{Q}\left|K(x, y)-K\left(x_{0}, y\right)\right|^{2} d y<\frac{\epsilon}{5}, \quad x \in V_{x_{0}} \cap Q .
$$

Para controlar a outra integral usamos a desigualdade,

$$
\begin{aligned}
\left|K(x, y)-K\left(x_{0}, y\right)\right|^{2} \leq & |K(x, y)|^{2}+\left|K\left(x_{0}, y\right)\right|^{2}+2|K(x, y)|\left|K\left(x_{0}, y\right)\right| \\
\leq & K(x, x) K(y, y)+K\left(x_{0}, x_{0}\right) K(y, y) \\
& +K\left(x_{0}, x_{0}\right)^{1 / 2} K(x, x)^{1 / 2} K(y, y) \\
\leq & 4 M K(y, y), \quad y \in D \backslash Q .
\end{aligned}
$$

Integrando e relembrando a nossa escolha de $r$, obtemos

$$
\int_{D \backslash Q}\left|K(x, y)-K\left(x_{0}, y\right)\right|^{2} d y \leq 4 M \int_{D \backslash Q} K(y, y) d y<\frac{4 \epsilon}{5} .
$$

Assim,

$$
\int_{D}\left|K(x, y)-K\left(x_{0}, y\right)\right|^{2} d y<\epsilon \quad x \in V_{x_{0}} \cap Q .
$$

O restante segue da desigualdade do início da prova e do fato de $K \in C_{o}(D \times D)$. 
Corolário 2.5.4. Nas condições do lema anterior, o operador integral $\mathcal{K}$ tem as seguintes propriedades: $\operatorname{Im} \mathcal{K} \subset C_{o}(D) \cap L^{2}(D)$,

$$
\|\mathcal{K}(\phi)\|_{C_{o}(D)} \leq \sqrt{M}\left(\int_{D} K(x, x) d x\right)^{1 / 2}\|\phi\|_{L^{2}(D)}, \quad \phi \in L^{2}(D),
$$

$e$

$$
\|\mathcal{K}(\phi)\|_{L^{2}(D)} \leq \int_{D} K(x, x) d x\|\phi\|_{L^{2}(D)}, \quad \phi \in L^{2}(D),
$$

onde $M=\max \{K(x, x): x \in D\}$.

Demonstração: Seja $\phi \in L^{2}(D)$. A desigualdade

$$
\begin{aligned}
\left|\mathcal{K}(\phi)(x)-\mathcal{K}(\phi)\left(x_{0}\right)\right| & =\left|\int_{D}\left[K(x, y)-K\left(x_{0}, y\right)\right] \phi(y) d y\right| \\
& \leq\left(\int_{D}\left|K(x, y)-K\left(x_{0}, y\right)\right|^{2} d y\right)^{1 / 2}\|\phi\|_{L^{2}(D)}, \quad \phi \in L^{2}(D),
\end{aligned}
$$

e o lema anterior justificam a continuidade de $\mathcal{K}(\phi)$ em $x_{0} \in D$. Como a função $x \in$ $D \mapsto K(x, x)$ é um elemento de $L^{1}(D)$, a estimativa

$$
\begin{aligned}
|\mathcal{K}(\phi)(x)|=\left|\int_{D} K(x, y) \phi(y) d y\right| & \leq\left(\int_{D}|K(x, y)|^{2} d y\right)^{1 / 2}\|\phi\|_{L^{2}(D)} \\
& \leq\left(K(x, x) \int_{D} K(y, y) d y\right)^{1 / 2}\|\phi\|_{L^{2}(D)} \\
& =K(x, x)^{1 / 2}\left(\int_{D} K(y, y) d y\right)^{1 / 2}\|\phi\|_{L^{2}(D)} .
\end{aligned}
$$

mostra que $\lim _{|x| \rightarrow \infty} \mathcal{K}(\phi)(x)=0$. As duas desigualdades do enunciado do corolário são obtidas diretamente desta mesma estimativa.

Teorema 2.5.5. Seja D um subconjunto fechado de $\mathbb{R}^{m}$. Se D tem medida estritamente positiva e $K \in \mathcal{A}_{o}(D)$, então valem as seguintes afirmações:

(i) $\operatorname{Im} \mathcal{K} \subset C_{o}(D) \cap L^{2}(D)$;

(ii) $K$ é representável por uma série $L^{2}(D \times D)$, absoluta e uniformemente convergente da forma

$$
K(x, y)=\sum_{n=1}^{\infty} \lambda_{n}(\mathcal{K}) \phi_{n}(x) \overline{\phi_{n}(y)}, \quad x, y \in D,
$$

sendo $\left\{\phi_{n}\right\}$ um conjunto $L^{2}(D)$-ortonormal e $\lambda_{n}(\mathcal{K}) \geq \lambda_{n}(\mathcal{K}) \geq \cdots \geq 0$.

Demonstração: Segue do Lema 2.5.3 e do Teorema 2.4.11. 
Teorema 2.5.6. Seja $D$ um subconjunto fechado de $\mathbb{R}^{m}$ com medida estritamente positiva e $K \in L^{2} P D(D)$ um núcleo suave. Então

$$
\operatorname{tr}(|\mathcal{K}|)=\operatorname{tr}(\mathcal{K})=\sum_{n=1}^{\infty} \lambda_{n}(\mathcal{K})=\int_{D} K(x, x) d x .
$$

Em particular, $\mathcal{K}$ é nuclear se, e somente se, $K \in \mathcal{A}_{o}(D)$.

Demonstração: Do Teorema 2.4.11, segue que a seqüência $\left\{\sum_{n=1}^{k} \lambda_{n}(\mathcal{K})\left|\phi_{n}(x)\right|^{2}\right\}$ é crescente, convergindo uniformemente para $K(x, x)$. Com isso podemos usar o Teorema da Convergência Monótona para deduzir que

$$
\int_{D} K(x, x) d x=\int_{D} \sum_{n=1}^{\infty} \lambda_{n}(\mathcal{K})\left|\phi_{n}(x)\right|^{2} d x=\sum_{n=1}^{\infty} \lambda_{n}(\mathcal{K}) \int_{D}\left|\phi_{n}(x)\right|^{2} d x=\sum_{n=1}^{\infty} \lambda_{n}(\mathcal{K}) .
$$

Segue que

$$
\operatorname{tr}(|\mathcal{K}|)=\operatorname{tr}(\mathcal{K})=\sum_{n=1}^{\infty} \lambda_{n}(\mathcal{K})=\int_{D} K(x, x) d x .
$$

A expressão acima é finita se, e somente se, $x \in D \mapsto K(x, x)$ é um elemento de $L^{1}(D)$. Nas condições do teorema, isto equivale a $K \in \mathcal{A}_{o}(D)$.

Observação 2.5.7. A condição $K \in C_{o}(D \times D)$ é essencial no Teorema 2.5.5, conforme o seguinte exemplo mostra. Seja $\phi_{n}=f_{n}+g_{n}$, onde

$$
f_{n}(x)=\frac{n^{2}+1}{a_{n}}\left(x-n-\frac{1}{2}+a_{n}\right) \chi_{\left[n-a_{n}+1 / 2, n+1 / 2\right]}(x)
$$

$\mathrm{e}$

$$
g_{n}(x)=\frac{n^{2}+1}{-a_{n}}\left(x-n-\frac{1}{2}-a_{n}\right) \chi_{\left[n+1 / 2, n+a_{n}+1 / 2\right]}(x),
$$

com $a_{n}=3 /\left(2\left(n^{2}+1\right)^{2}\right)$. Os gráficos de $\phi_{1}$ e $\phi_{2}$ estão mostrados na figura seguinte.

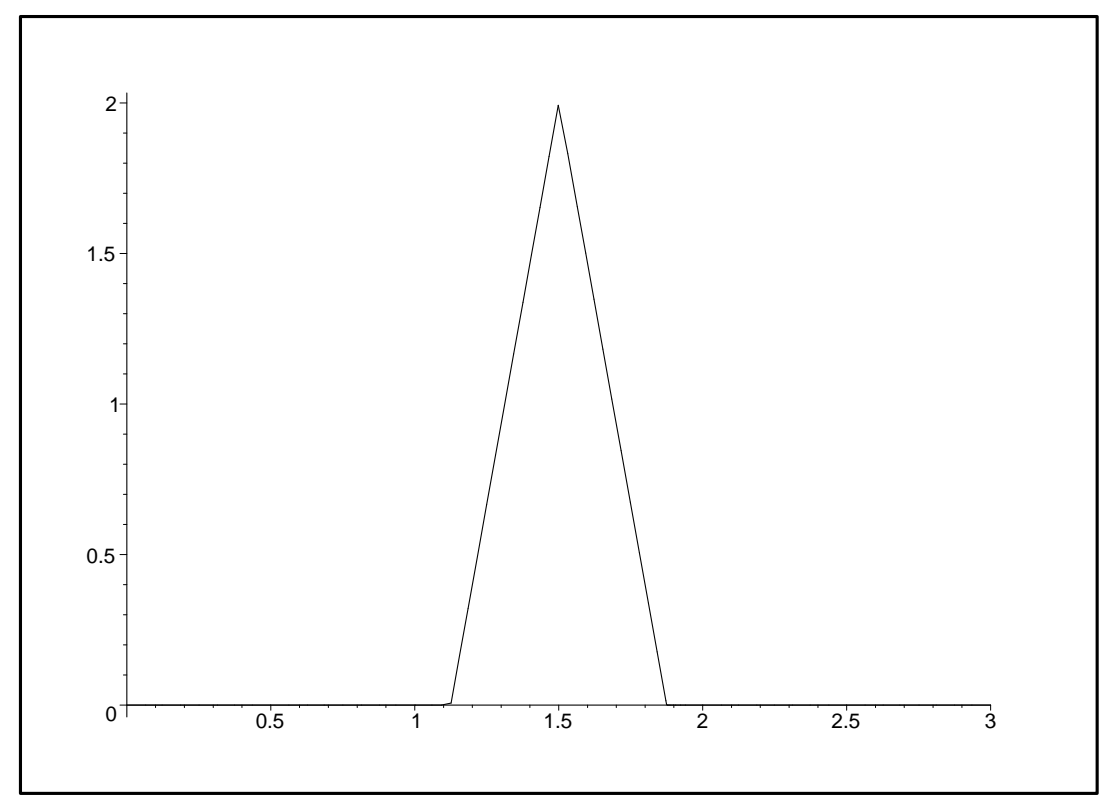




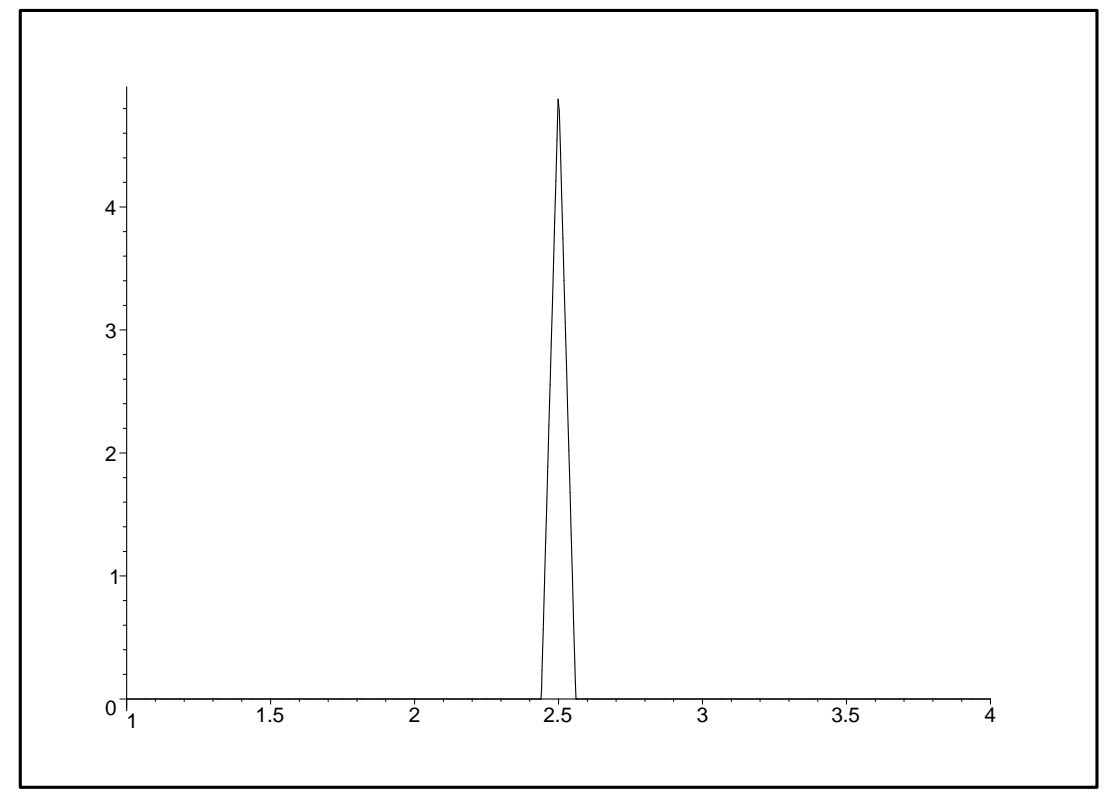

É fácil ver que $\left\{\phi_{n}\right\}$ é um conjunto $L^{2}(\mathbb{R})$-ortonormal. Tomando $\lambda_{n}(\mathcal{K})=1 / n^{2}$, o núcleo

$$
K(x, y):=\sum_{1}^{\infty} \lambda_{n}(\mathcal{K}) \phi_{n}(x) \overline{\phi_{n}(y)}, \quad x, y \in \mathbb{R},
$$

é tal que $K \in L^{2} P D(\mathbb{R}) \cap C(\mathbb{R} \times \mathbb{R})$ e $x \in \mathbb{R} \mapsto K(x, x)$ é um elemento de $L^{1}(\mathbb{R})$. Entretanto, a série acima não converge uniformemente para $K$ pois a soma parcial da série é limitada enquanto que $K$ não é.

A próxima definição permite uma reformulação de parte dos resultados provados anteriormente.

Definição 2.5.8. Sejam $X$ um subconjunto de $\mathbb{R}^{m}, \alpha \in \mathbb{R}$ e $f: X \rightarrow \mathbb{R}$. Dizemos que $f(x)=O\left(|x|^{\alpha}\right)$ se existem $r, M>0$ tais que $|f(x)| \leq M|x|^{\alpha}, x \in X \backslash[-r, r]^{m}$. Dizemos que $f(x)=o\left(|x|^{\alpha}\right)$ se, dado $M>0$, existir $r=r(M)>0$ tal que $|f(x)| \leq M|x|^{\alpha}$, $x \in X \backslash[-r, r]^{m}$.

Corolário 2.5.9. Seja $D$ um subconjunto fechado de $\mathbb{R}^{m}$. Se $D$ tem medida estritamente positiva, $K \in L^{2} P D(D) \cap C(D \times D)$ e $K(x, x)=O\left(|x|^{-\beta}\right)$, para algum $\beta>m$, então todas as afirmações do Teorema 2.5 .5 são válidas.

Demonstração: Pelo Teorema 1.3 .12 e pela continuidade de $K$ segue que a função $x \in D \mapsto K(x, x)$ é um elemento de $L^{1}(D)$. A hipótese adicional sobre $K$ implica que $K \in C_{o}(D \times D)$. Portanto, $K \in \mathcal{A}_{o}(D)$ e o Teorema 2.5.5 é aplicável.

O lema abaixo será útil na próxima seção. 
Lema 2.5.10. Sejam $D$ um subconjunto fechado de $\mathbb{R}^{m}$ com medida estritamente positiva e $K \in L^{2} P D(D) \cap C_{o}(D \times D)$. Se $x \in D \mapsto K^{1 / 2}(x, x)$ é um elemento de $L^{1}(D)$, então as seguintes afirmações são verdadeiras:

(i) $K \in \mathcal{A}_{o}(D)$ e, em particular, as conclusões do Teorema 2.5.5 valem;

(ii) $\operatorname{Im} \mathcal{K} \subset C_{o}(D) \cap L^{2}(D) \cap L^{1}(D) e$

$$
\|\mathcal{K}(\phi)\|_{L^{1}(D)} \leq\left(\int_{D} K^{1 / 2}(x, x) d x\right)\left[\int_{D} K(x, x) d x\right]^{1 / 2}\|\phi\|_{L^{2}(D)} .
$$

(iii) Se $\lambda_{n}(\mathcal{K})$ é não-nulo, então

$$
\left\|\phi_{n}\right\|_{L^{1}(D)} \leq \frac{1}{\lambda_{n}(\mathcal{K})} \int_{D} K^{1 / 2}(x, x) d x\left[\int_{D} K(x, x) d x\right]^{1 / 2} .
$$

(iv) O núcleo $K$ é um elemento de $L^{1}(D \times D)$,

$$
\|K\|_{L^{1}(D \times D)} \leq\left(\int_{D} K^{1 / 2}(x, x) d x\right)^{2}
$$

e a série $\sum_{n=1}^{\infty} \lambda_{n}(\mathcal{K}) \phi_{n} \otimes \overline{\phi_{n}}$ é $L^{1}(D \times D)$-convergente para $K$.

Demonstração: $(i)$ Se $M:=\max \left\{K^{1 / 2}(x, x): x \in D\right\}<\infty$, então

$$
\int_{D} K(x, x) d x=\int_{D} K^{1 / 2}(x, x) K^{1 / 2}(x, x) d x \leq M \int_{D} K^{1 / 2}(x, x) d x .
$$

Logo, $x \in D \mapsto K(x, x)$ é um elemento de $L^{1}(D)$. Portanto, $K \in \mathcal{A}_{0}(D)$.

(ii) Se $\phi \in L^{2}(D)$, usando a desigualdade de Cauchy-Schwarz, obtemos

$$
\begin{aligned}
\int_{D}|\mathcal{K}(\phi)(x)| d x & =\int_{D}\left|\int_{D} K(x, y) \phi(y) d y\right| d x \\
& \leq \int_{D}\left(\int_{D}|K(x, y)|^{2} d y\right)^{1 / 2}\left(\int_{D}|\phi(y)|^{2} d y\right)^{1 / 2} d x \\
& \leq \int_{D}\left(\int_{D} K(x, x) K(y, y) d y\right)^{1 / 2}\left(\int_{D}|\phi(y)|^{2} d y\right)^{1 / 2} d x \\
& =\left(\int_{D} K^{1 / 2}(x, x) d x\right)\left(\int_{D} K(y, y) d y\right)^{1 / 2}\|\phi\|_{L^{2}(D)}
\end{aligned}
$$

(iii) Basta notar que se $\phi_{n}$ é uma autofunção associada a um autovalor não-nulo $\lambda_{n}(\mathcal{K})$, então o item (ii) implica que $\mathcal{K}\left(\phi_{n}\right)=\lambda_{n}(\mathcal{K}) \phi_{n} \in L^{1}(D)$, bem como a desigualdade anunciada.

(iv) A desigualdade

$$
\int_{D} \int_{D}|K(x, y)| d x d y \leq \int_{D} K^{1 / 2}(x, x) K^{1 / 2}(y, y) d x d y=\left(\int_{D} K^{1 / 2}(x, x) d x\right)^{2}
$$


revela que $K \in L^{1}(D \times D)$. Para completar a prova do item, defina

$$
g_{n}(x, y)=\sum_{j=n}^{\infty} \lambda_{j}(\mathcal{K})\left|\phi_{j}(x)\right|\left|\phi_{j}(y)\right|, \quad x, y \in D, \quad n=1,2, \ldots
$$

A desigualdade de Cauchy-Schwarz implica que

$$
\begin{aligned}
g_{n}(x, y) & =\sum_{j=n}^{\infty} \lambda_{j}(\mathcal{K})\left|\phi_{j}(x)\right|\left|\phi_{j}(y)\right| \\
& \leq\left(\sum_{j=n}^{\infty} \lambda_{j}(\mathcal{K})\left|\phi_{j}(x)\right|^{2}\right)^{1 / 2}\left(\sum_{j=n}^{\infty} \lambda_{j}(\mathcal{K})\left|\phi_{j}(y)\right|^{2}\right)^{1 / 2} \\
& \leq K^{1 / 2}(x, x) K^{1 / 2}(y, y), \quad x, y \in D
\end{aligned}
$$

e segue que a seqüência $\left\{g_{n}\right\}$ é não-crescente e converge pontualmente para 0. Logo, aplicando o Teorema da Convergência Monótona na seqüência $\left\{g_{1}-g_{n}\right\}$ obtemos

$$
\int_{D} \int_{D}\left|K(x, y)-\sum_{j=1}^{n} \lambda_{j}(\mathcal{K}) \phi_{j}(x) \overline{\phi_{j}(y)}\right| d x d y \leq \int_{D} \int_{D} g_{n}(x, y) d x d y \rightarrow 0 .
$$

Portanto, a série $\sum_{j=1}^{\infty} \lambda_{j}(\mathcal{K}) \phi_{j} \otimes \overline{\phi_{j}}$ é $L^{1}(D \times D)$-convergente para $K$.

Corolário 2.5.11. Seja $D$ um subconjunto compacto de $\mathbb{R}^{m}$ com medida estritamente positiva. Se $K \in L^{2} P D(D) \cap C(D \times D)$, então as conclusões do Teorema 2.5.5 e do Lema 2.5.10 são válidas.

Demonstração: Basta notar que nas condições do corolário, as hipóteses do Lema 2.5.10 estão satisfeitas.

O corolário abaixo é uma versão mais fraca do Teorema 2.5.5.

Corolário 2.5.12. Sejam $D$ um subconjunto fechado de $\mathbb{R}^{m}$ com medida estritamente positiva e $K \in L^{2} P D(D) \cap C(D \times D)$. Se $K(x, x)=O\left(|x|^{-\beta}\right)$ para algum $\beta>2 m$, então todas as afirmações do Teorema 2.5.5 e do Lema 2.5.10 são verdadeiras.

Demonstração: Basta notar que a condição $K^{1 / 2}(x, x)=O\left(|x|^{-\beta / 2}\right)$ implica que a função $x \in D \mapsto K^{1 / 2}(x, x)$ é um elemento de $L^{1}(D)$ e que $K \in C_{o}(D \times D)$.

Observação 2.5.13. Seja

$$
\phi(x):=\left\{\begin{array}{ll}
\left(x \pi^{1 / 2}\right)^{-1} \operatorname{sen} x & \text { se } x \neq 0 \\
1 & \text { se } x=0
\end{array} .\right.
$$

É fácil ver que $\|\phi\|_{L^{2}(\mathbb{R})}=1$. Definindo

$$
K(x, y):=\pi \phi(x) \overline{\phi(y)}, \quad x, y \in \mathbb{R}
$$


vemos que $K \in L^{2} P D(\mathbb{R}) \cap C_{o}(\mathbb{R} \times \mathbb{R})$. Ainda, $\mathcal{K}(\phi)=\pi \phi$, ou seja, $\phi$ é autofunção de $\mathcal{K}$ associada ao autovalor $\pi$. Como $\mathcal{K}$ tem posto $1, \phi$ é a única autofunção de $\mathcal{K}$ associada ao único autovalor não-nulo $\pi$. Além disso, $x \in \mathbb{R} \mapsto K(x, x)$ é um elemento de $L^{1}(\mathbb{R})$, enquanto que $x \in \mathbb{R} \mapsto K^{1 / 2}(x, x)$ não é um elemento de $L^{1}(\mathbb{R})$. Portanto, como $\phi \notin L^{1}(\mathbb{R})$, segue que $\operatorname{Im} \mathcal{K} \not \subset L^{1}(\mathbb{R})$. Em particular, $K \notin L^{1}(\mathbb{R})$ e a série que define $K$ (que neste caso possui um único termo) não converge em $L^{1}(\mathbb{R} \times \mathbb{R})$. Assim, este exemplo mostra que a hipótese $x \in D \mapsto K^{1 / 2}(x, x) \in L^{1}(D)$ do Lema 2.5.10 é essencial.

Observação 2.5.14. Note que a estimativa para a norma de $K$ em $L^{1}(D \times D)$, dada pelo Lema 2.5.10, é atingida quando $K(x, y)=\phi(x) \overline{\phi(y)}$, com $\phi(x) \geq 0$ e $\phi \in C_{o}(D) \cap$ $L^{1}(D) \cap L^{2}(D)$, ou seja, a estimativa não é passível de melhora.

\subsection{Núcleos $L^{2}$-positivos definidos e a Transformada de Fourier}

Nesta seção investigamos possíveis relações entre núcleos $L^{2}$-positivos definidos e sua transformada de Fourier.

A principal conexão entre os dois conceitos tem origem no teorema abaixo ([39]).

Teorema 2.6.1 (Bochner). Um núcleo contínuo da forma $(x, y) \in \mathbb{R}^{m} \times \mathbb{R}^{m} \mapsto$ $f(x-y)$ é um elemento de $P D\left(\mathbb{R}^{m}\right)$ se, e somente se, $f$ é a transformada de Fourier de uma medida $\sigma$ positiva e finita, isto é,

$$
f(x)=\int_{\mathbb{R}^{m}} e^{i y \cdot x} d \sigma(y), \quad x \in \mathbb{R}^{m} .
$$

Comecemos nosso estudo formalizando no contexto $X=\mathbb{R}^{m}$, o funcional linear implícito na Definição 2.2.1.

Definição 2.6.2. Seja $\phi \in L^{2}\left(\mathbb{R}^{m}\right)$. A aplicação $T_{\phi}: L^{2}\left(\mathbb{R}^{m} \times \mathbb{R}^{m}\right) \rightarrow \mathbb{C}$ dada por

$$
T_{\phi}(K)=\langle K, \phi \otimes \bar{\phi}\rangle=\int_{\mathbb{R}^{m}} \int_{\mathbb{R}^{m}} K(x, y) \overline{\phi(x)} \phi(y) d x d y, \quad K \in L^{2}\left(\mathbb{R}^{m} \times \mathbb{R}^{m}\right),
$$

é o funcional linear contínuo determinado por $\phi$.

No lema abaixo utilizamos a notação introduzida na Seção 1.7. Se $K \in L^{2}\left(\mathbb{R}^{m} \times \mathbb{R}^{m}\right)$, então $\hat{K}$ é o núcleo obtido via a Observação 1.7.6 enquanto que $\tilde{K}$ é o núcleo dado pela fórmula $\tilde{K}(u, v):=\hat{K}(u,-v), u, v \in \mathbb{R}^{m}$.

Lema 2.6.3. Nas condições do parágrafo anterior, a seguinte fórmula vale: $T_{\phi}(K)=$ $T_{\hat{\phi}}(\tilde{K}), \phi \in L^{2}\left(\mathbb{R}^{m}\right)$. 
Demonstração: Seja $\phi \in L^{2}\left(\mathbb{R}^{m}\right)$. Pela Identidade de Parseval já temos que

$$
T_{\phi}(K)=\langle K, \phi \otimes \bar{\phi}\rangle=\langle\hat{K}, \widehat{\phi \otimes \bar{\phi}}\rangle .
$$

Lembrando o Lema 1.7.5 e o Lema 1.7.7 e utilizando o Teorema da Mudança de Variável em Integrais, obtemos

$$
\int_{\mathbb{R}^{m}} \int_{\mathbb{R}^{m}}|\hat{K}(u,-v)|^{2} d u d v=\int_{\mathbb{R}^{m}} \int_{\mathbb{R}^{m}}|\hat{K}(u, v)|^{2} d u d v=\|\hat{K}\|_{L^{2}\left(\mathbb{R}^{m} \times \mathbb{R}^{m}\right)}^{2}
$$

ou seja, $\tilde{K} \in L^{2}\left(\mathbb{R}^{m} \times \mathbb{R}^{m}\right)$. Também,

$T_{\phi}(K)=\int_{\mathbb{R}^{m}} \int_{\mathbb{R}^{m}} \hat{K}(u, v) \overline{\hat{\phi}(u)} \hat{\phi}(-v) d u d v=\int_{\mathbb{R}^{m}} \int_{\mathbb{R}^{m}} \hat{K}(u,-v) \overline{\hat{\phi}(u)} \hat{\phi}(v) d u d v=T_{\hat{\phi}}(\tilde{K})$,

já que

$$
\widehat{\phi \otimes \bar{\phi}}(u, v)=\hat{\phi}(u) \hat{\bar{\phi}}(v)=\hat{\phi(u)} \overline{\hat{\phi}(-v)}, \quad u, v \in \mathbb{R}^{m}
$$

Isto completa a prova.

O lema acima nos fornece a seguinte equivalência.

Teorema 2.6.4. Seja $K \in L^{2}\left(\mathbb{R}^{m} \times \mathbb{R}^{m}\right)$. Então $K \in L^{2} P D\left(\mathbb{R}^{m}\right)$ se, e somente se, $\tilde{K} \in L^{2} P D\left(\mathbb{R}^{m}\right)$.

Demonstração: Pelo Lema 2.6.3, a condição $T_{\phi}(K) \geq 0, \phi \in L^{2}\left(\mathbb{R}^{m}\right)$, equivale a $T_{\hat{\phi}}(\tilde{K}) \geq 0, \phi \in L^{2}\left(\mathbb{R}^{m}\right)$. Como a transformada de Fourier é um isomorfismo, o resultado segue.

Corolário 2.6.5. Seja $K \in L^{2}\left(\mathbb{R}^{m} \times \mathbb{R}^{m}\right)$. Então $K \in L^{2} P D\left(\mathbb{R}^{m}\right)$ se, e somente se, existe $G \in L^{2} P D\left(\mathbb{R}^{m}\right)$ tal que $K=\tilde{G}$.

Demonstração: Pelo Teorema de Plancherel, existe $H \in L^{2}\left(\mathbb{R}^{m} \times \mathbb{R}^{m}\right)$ tal que $K=\hat{H}$. Tomemos agora $\left\{H_{n}\right\} \subset L^{1}\left(\mathbb{R}^{m} \times \mathbb{R}^{m}\right) \cap L^{2}\left(\mathbb{R}^{m} \times \mathbb{R}^{m}\right)$ convergindo para $H$ em $L^{2}\left(\mathbb{R}^{m} \times\right.$ $\left.\mathbb{R}^{m}\right)$. Definindo $G(x, y):=H(x,-y), x, y \in \mathbb{R}^{m}$ e usando o Teorema da Mudança de Variaveis em Integrais, vemos que $G_{n}(x, y):=H_{n}(x,-y)$ converge para $G$ em $L^{2}\left(\mathbb{R}^{m} \times\right.$ $\left.\mathbb{R}^{m}\right)$. Aplicando a definição da transformada de Fourier $\left(\right.$ em $L^{1}\left(\mathbb{R}^{m} \times \mathbb{R}^{m}\right)$ ) concluímos que $\hat{G}_{n}(u,-v)=\hat{H}_{n}(u, v)$. Da continuidade da transformada de Fourier, segue que $\tilde{G}(u, v)=\hat{G}(u,-v)=\hat{H}(u, v)=K(u, v)$, quase sempre. Utilizando o Teorema 2.6.4 temos o resultado.

Corolário 2.6.6. Seja $K \in L^{2}\left(\mathbb{R}^{m} \times \mathbb{R}^{m}\right)$. Defina $K_{(1)}=K$ e, indutivamente, $K_{(n)}=$ $\tilde{K}_{(n-1)}, n=2,3, \ldots$ Se $K \in L^{2} P D\left(\mathbb{R}^{m}\right)$, então $K_{(n)} \in L^{2} P D\left(\mathbb{R}^{m}\right), n=1,2, \ldots$. 
No que segue, denotamos pela letra $B$ a transformação bilinear $B: L^{2}\left(\mathbb{R}^{m} \times \mathbb{R}^{m}\right) \times$ $L^{2}\left(\mathbb{R}^{m}\right) \rightarrow L^{2}\left(\mathbb{R}^{m}\right)$ dada por

$$
B(K, \phi)=\mathcal{K}(\phi), \quad K \in L^{2}\left(\mathbb{R}^{m} \times \mathbb{R}^{m}\right), \quad \phi \in L^{2}\left(\mathbb{R}^{m}\right) .
$$

Se normalizarmos $L^{2}\left(\mathbb{R}^{m} \times \mathbb{R}^{m}\right) \times L^{2}\left(\mathbb{R}^{m}\right)$ com a norma do máximo, então $B$ é contínua. Ainda,

$$
\|B(K, \phi)\|_{L^{2}\left(\mathbb{R}^{m}\right)} \leq\|K\|_{L^{2}\left(\mathbb{R}^{m} \times \mathbb{R}^{m}\right)}\|\phi\|_{L^{2}\left(\mathbb{R}^{m}\right)}, \quad K \in L^{2}\left(\mathbb{R}^{m} \times \mathbb{R}^{m}\right), \quad \phi \in L^{2}\left(\mathbb{R}^{m}\right) .
$$

Seja $K \in L^{2}\left(\mathbb{R}^{m} \times \mathbb{R}^{m}\right)$ e suponha que $K(x, \cdot) \in L^{2}\left(\mathbb{R}^{m}\right)$, para algum $x \in \mathbb{R}^{m}$. Neste caso, podemos denotar por $\hat{K}_{2}(x, y)$ a transformada de Fourier da função $y \in \mathbb{R}^{m} \mapsto$ $K(x, y)$. Do Teorema de Fubini segue que $K(x, \cdot) \in L^{2}\left(\mathbb{R}^{m}\right)$ para todo $x \in \mathbb{R}^{m} \backslash X$, com $X$ de medida nula. Utilizando a Identidade de Parseval e o Teorema de Tonelli, temos que

$$
\int_{\mathbb{R}^{m} \backslash X} \int_{\mathbb{R}^{m}}\left|\hat{K}_{2}(x, y)\right|^{2} d y d x=\int_{\mathbb{R}^{m} \backslash X} \int_{\mathbb{R}^{m}}|K(x, y)|^{2} d y d x=\int_{\mathbb{R}^{m}} \int_{\mathbb{R}^{m}}|K(x, y)|^{2} d y d x
$$

Após redefinição em um conjunto de medida nula a função $(x, y) \in R^{m} \times \mathbb{R}^{m} \mapsto \hat{K}_{2}(x, y)$ é um elemento de $L^{2}\left(R^{m} \times \mathbb{R}^{m}\right)$.

Lema 2.6.7. Nas condições acima:

(i) Se $K(x, \cdot) \in L^{2}(D)$, então

$$
B(K, \phi)(x)=B\left(\hat{K}_{2}, \overline{\hat{\phi}}\right)(x), \quad \phi \in L^{2}\left(\mathbb{R}^{m}\right)
$$

(ii) Vale a fórmula

$$
\left.B \widehat{\left(\hat{K}_{2}, \psi\right.}\right)=B(\hat{K}, \psi), \quad \psi \in L^{2}\left(\mathbb{R}^{m}\right)
$$

Demonstração: Para o item $(i)$ utilizamos a Identidade de Parseval para obter,

$$
\begin{aligned}
B(K, \phi)(x) & =\int_{\mathbb{R}^{m}} K(x, y) \phi(y) d y \\
& =\int_{\mathbb{R}^{m}} \hat{K}_{2}(x, y) \overline{\overline{\phi(y)}} d y \\
& =B\left(\hat{K}_{2}, \overline{\bar{\phi}}\right)(x), \quad x \in \mathbb{R}^{m} .
\end{aligned}
$$

Para (ii), usamos o Teorema 1.3.2 para obter seqüências $\left\{K^{n}\right\} \subset C\left(\mathbb{R}^{m} \times \mathbb{R}^{m}\right)$ e $\left\{\psi_{n}\right\} \subset C\left(\mathbb{R}^{m}\right)$ de funções com suporte compacto de modo que $\left\{K^{n}\right\}$ converge para 
$K$ em $L^{2}\left(\mathbb{R}^{m} \times \mathbb{R}^{m}\right)$ e $\left\{\psi_{n}\right\}$ converge para $\psi$ em $L^{2}\left(\mathbb{R}^{m}\right)$. Como $K^{n} \in L^{2}\left(\mathbb{R}^{m} \times \mathbb{R}^{m}\right) \cap$ $L^{1}\left(\mathbb{R}^{m} \times \mathbb{R}^{m}\right)$,

$$
B\left(\hat{K}^{n}, \psi_{n}\right)(x)=\int_{\mathbb{R}^{m}}\left(\int_{\mathbb{R}^{m}} \int_{\mathbb{R}^{m}} K^{n}(u, v) e^{-2 \pi i(u, v) \cdot(x, y)} d u d v\right) \psi_{n}(y) d y
$$

Observando que

$$
\left|K^{n}(u, v) e^{-2 \pi i(u, v) \cdot(x, y)} \psi_{n}(y)\right|=\left|K^{n}(u, v) \psi_{n}(y)\right|, \quad u, v, y \in \mathbb{R}^{m}
$$

e utilizando o Teorema de Fubini, a equação acima toma a forma

$$
\begin{aligned}
B\left(\hat{K}^{n}, \psi_{n}\right)(x) & =\int_{\mathbb{R}^{m}}\left(\int_{\mathbb{R}^{m}}\left(\int_{\mathbb{R}^{m}} K^{n}(u, v) e^{-2 \pi i v \cdot y} d v\right) \psi_{n}(y) d y\right) e^{-2 \pi i u \cdot x} d u \\
& =\int_{\mathbb{R}^{m}}\left(\int_{\mathbb{R}^{m}} \hat{K}_{2}^{n}(u, y) \psi_{n}(y) d y\right) e^{-2 \pi i x \cdot u} d u \\
& =\int_{\mathbb{R}^{m}} B\left(\hat{K}_{2}^{n}, \psi_{n}\right)(u) e^{-2 \pi i x \cdot u} d u,
\end{aligned}
$$

pois $K^{n}(u, \cdot) \in L^{1}\left(\mathbb{R}^{m}\right), u \in \mathbb{R}^{m}$. Mas

$$
\begin{aligned}
& \int_{\mathbb{R}^{m}}\left|B\left(\hat{K_{2}^{n}}, \psi_{n}\right)(u)\right| d u=\int_{\mathbb{R}^{m}}\left|\int_{\mathbb{R}^{m}}\left(\int_{\mathbb{R}^{m}} K^{n}(u, v) e^{-2 \pi i v \cdot y} d v\right) \psi_{n}(y) d y\right| d u \\
& \leq \int_{\mathbb{R}^{m}}\left(\int_{\mathbb{R}^{m}}\left(\int_{\mathbb{R}^{m}}\left|K^{n}(u, v)\right| d v\right)\left|\psi_{n}(y)\right| d y\right) d u \text {. }
\end{aligned}
$$

Como $K^{n}$ e $\psi_{n}$ são contínuas e possuem suporte compacto segue que $B\left(\hat{K}_{2}^{n}, \psi_{n}\right) \in$ $L^{1}\left(\mathbb{R}^{m}\right)$. Portanto,

$$
\left.B\left(\hat{K}^{n}, \psi_{n}\right)(x)=B \widehat{\left(\hat{K}_{2}^{n}, \psi_{n}\right.}\right)(x) .
$$

Agora, utilizando a continuidade de $B$ e da transformada de Fourier e lembrando que $\lim _{n \rightarrow \infty} K^{n}(x,)=.K(x,$.$\left.) , quase sempre, obtemos B(\hat{K}, \psi)=\widehat{B\left(\hat{K}_{2}, \psi\right.}\right)$.

A próxima proposição analisa a possibilidade de representarmos a transformada de Fourier de um núcleo $K \in L^{2} P D\left(\mathbb{R}^{m}\right)$ por uma série.

Proposição 2.6.8. Se $K \in L^{2} P D\left(\mathbb{R}^{m} \times \mathbb{R}^{m}\right)$ e $\tilde{K}(u, v)=\hat{K}(u,-v), u, v \in \mathbb{R}^{m}$, então as seguintes afirmações são verdadeiras:

(i) Os operadores positivos $\mathcal{K}$ e $\tilde{\mathcal{K}}$ possuem o mesmo espectro, isto é, os mesmos autovalores com as mesmas multiplicidades;

(ii) Se $\phi_{n}$ é uma autofunção de $\mathcal{K}$, associada ao autovalor $\lambda_{n}(\mathcal{K})$, então $\hat{\phi}_{n}$ é uma autofunção de $\tilde{\mathcal{K}}$, associada ao autovalor $\lambda_{n}(\mathcal{K})$ e reciprocamente;

(iii) Se

$$
K=\sum_{n=1}^{\infty} \lambda_{n}(\mathcal{K}) \phi_{n} \otimes \overline{\phi_{n}}
$$


com convergência em $L^{2}\left(\mathbb{R}^{m} \times \mathbb{R}^{m}\right)$, onde $\left\{\phi_{n}\right\}$ é $L^{2}\left(\mathbb{R}^{m}\right)$-ortonormal, então $\tilde{K}$ possui uma expansão $L^{2}\left(\mathbb{R}^{m} \times \mathbb{R}^{m}\right)$-convergente na forma

$$
\tilde{K}=\sum_{n=1}^{\infty} \lambda_{n}(\mathcal{K}) \hat{\phi}_{n} \otimes \overline{\hat{\phi}_{n}},
$$

onde $\left\{\hat{\phi}_{n}\right\}$ é $L^{2}\left(\mathbb{R}^{m}\right)$-ortonormal;

(iv) Vale a recíproca do item anterior.

Demonstração: Note que $\phi_{n} \in L^{2}\left(\mathbb{R}^{m}\right)$ é uma autofunção de $\mathcal{K}$ associada a $\lambda_{n}(\mathcal{K})$ se, e somente se,

$$
\left\|\mathcal{K}\left(\phi_{n}\right)\right\|_{L^{2}\left(\mathbb{R}^{m}\right)}^{2}-\lambda_{n}(\mathcal{K})\left\langle\mathcal{K}\left(\phi_{n}\right), \phi_{n}\right\rangle-\lambda_{n}(\mathcal{K})\left\langle\phi_{n}, \mathcal{K}\left(\phi_{n}\right)\right\rangle+\lambda_{n}(\mathcal{K})^{2}\left\|\phi_{n}\right\|_{L^{2}\left(\mathbb{R}^{m}\right)}^{2}=0 .
$$

Parte da prova consiste em reescrever a identidade acima. Utilizando os lemas 2.6.3 e 2.6.7, temos que

$$
\left\langle\mathcal{K}\left(\phi_{n}\right), \phi_{n}\right\rangle=T_{\phi_{n}}(K)=T_{\hat{\phi}_{n}}(\tilde{K})=\left\langle\tilde{\mathcal{K}}\left(\hat{\phi_{n}}\right), \hat{\phi_{n}}\right\rangle
$$

e

$$
\begin{array}{r}
\left\|\mathcal{K}\left(\phi_{n}\right)\right\|_{L^{2}\left(\mathbb{R}^{m}\right)}^{2}=\left\|B\left(K, \phi_{n}\right)\right\|_{L^{2}\left(\mathbb{R}^{m}\right)}^{2}=\left\|B\left(\hat{\hat{K}_{2}}, \overline{\hat{\phi_{n}}}\right)\right\|_{L^{2}\left(\mathbb{R}^{m}\right)}^{2} \\
\left.=\| B \overline{\left(\hat{\hat{K}_{2}}, \overline{\hat{\phi_{n}}}\right.}\right)\left\|_{L^{2}\left(\mathbb{R}^{m}\right)}^{2}=\right\| B\left(\hat{K}, \overline{\hat{\phi_{n}}}\right)\left\|_{L^{2}\left(\mathbb{R}^{m}\right)}^{2}=\right\| \tilde{\mathcal{K}}\left(\hat{\phi_{n}}\right) \|_{L^{2}\left(\mathbb{R}^{m}\right)}^{2} .
\end{array}
$$

Como $\left\|\phi_{n}\right\|_{L^{2}\left(\mathbb{R}^{m}\right)}^{2}=\left\|\hat{\phi}_{n}\right\|_{L^{2}\left(\mathbb{R}^{m}\right)}^{2}$, os cálculos acima nos permitem concluir, via Identidade de Parseval, que

$$
\left\|\hat{\mathcal{K}}\left(\hat{\phi}_{n}\right)\right\|_{L^{2}\left(\mathbb{R}^{m}\right)}^{2}-\lambda_{n}(\mathcal{K})\left\langle\hat{\mathcal{K}}\left(\hat{\phi}_{n}\right), \hat{\phi}_{n}\right\rangle-\lambda_{n}(\mathcal{K})\left\langle\hat{\phi}_{n}, \hat{\mathcal{K}}\left(\hat{\phi}_{n}\right)\right\rangle+\lambda_{n}(\mathcal{K})^{2}\left\|\hat{\phi}_{n}\right\|_{L^{2}\left(\mathbb{R}^{m}\right)}^{2}=0
$$

Isto prova a primeira metade de $(i)$ e de $(i i)$. As segundas metades são feitas de maneira análoga. Para provarmos (iii) utilizamos a Observação 1.7.6 para obter

$$
\overline{\tilde{K}(u, v)}=\overline{\hat{K}(u,-v)}=\overline{\hat{K(-u, v)}}=\hat{K}(v,-u)=\tilde{K}(v, u), \quad u, v \in \mathbb{R}^{m} \text { (q.s.), }
$$

uma vez que a positividade de $\mathcal{K}$ implica que $K$ é hermitiano quase sempre. Logo, $\tilde{K}$ é hermitiano e o Teorema 1.6.6 implica que $\tilde{K}$ possui uma representação $L^{2}\left(\mathbb{R}^{m} \times \mathbb{R}^{m}\right)$ convergente na forma

$$
\tilde{K}=\sum_{n=1}^{\infty} \lambda_{n}(\tilde{\mathcal{K}}) \psi_{n} \otimes \overline{\psi_{n}},
$$

onde $\left\{\psi_{n}\right\}$ é uma base $L^{2}\left(\mathbb{R}^{m}\right)$-ortonormal de autofunções de $\tilde{\mathcal{K}}$. Note que por $(i)$, necessariamente $\lambda_{n}(\tilde{\mathcal{K}})=\lambda_{n}(\mathcal{K})$, enquanto que por $(i i), \psi_{n}=\hat{\phi}_{n}, n=1,2, \ldots$ A prova do item (iv) está implícita na argumentação acima.

O resultado principal desta seção é o teorema abaixo. 
Teorema 2.6.9. Seja $K \in \mathcal{A}_{o}\left(\mathbb{R}^{m}\right)$ tal que a função $x \in \mathbb{R}^{m} \mapsto K^{1 / 2}(x, x)$ é um elemento de $L^{1}\left(\mathbb{R}^{m}\right)$. Então, além das conclusões do Lema 2.5.10, as seguintes afirmações são válidas:

(i) O núcleo $\tilde{K}$ é um elemento de $\mathcal{A}_{o}\left(\mathbb{R}^{m}\right)$ e possui uma expansão $L^{2}\left(\mathbb{R}^{m} \times \mathbb{R}^{m}\right)$, absoluta e uniformemente convergente da forma

$$
\tilde{K}(u, v)=\sum_{n=1}^{\infty} \lambda_{n}(\mathcal{K}) \hat{\phi}_{n}(u) \overline{\hat{\phi}_{n}(v)}
$$

onde cada $\lambda_{n}(\mathcal{K})$ é um autovalor do operador integral $\mathcal{K}$ e cada $\hat{\phi}_{n}$ é a transformada de Fourier da autofunção $\phi_{n}$ correspondente. O conjunto $\left\{\hat{\phi}_{n}\right\}$ é $L^{2}\left(\mathbb{R}^{m}\right)$-ortonormal, cada $\hat{\phi}_{n}$ é autofunção do operador $\tilde{\mathcal{K}}$ cuja imagem é um subconjunto de $C_{o}\left(\mathbb{R}^{m}\right) \cap L^{2}\left(\mathbb{R}^{m}\right)$; (ii) O operador $\tilde{\mathcal{K}}$ é nuclear e

$$
\operatorname{tr}(|\tilde{\mathcal{K}}|)=\operatorname{tr}(\tilde{\mathcal{K}})=\operatorname{tr}(\mathcal{K})=\sum_{n=1}^{\infty} \lambda_{n}(\mathcal{K})=\int_{\mathbb{R}^{m}} K(x, x) d x=\int_{\mathbb{R}^{m}} \tilde{K}(u, u) d u
$$

Demonstração: Essencialmente, a demonstração consiste em mostrar que $\tilde{K}$ satisfaz às hipóteses do Teorema 2.5.5. Do Teorema 2.6.4 e do Lema de Riemann-Lebesgue, segue que $\tilde{K} \in L^{2} P D\left(\mathbb{R}^{m}\right) \cap C_{o}\left(\mathbb{R}^{m} \times \mathbb{R}^{m}\right)$, pois $K \in L^{2} P D\left(\mathbb{R}^{m}\right) \cap L^{1}\left(\mathbb{R}^{m} \times \mathbb{R}^{m}\right)$. Mostremos que $u \in \mathbb{R}^{m} \mapsto \tilde{K}(u, u)$ está em $L^{1}\left(\mathbb{R}^{m}\right)$. Como as condições do Lema 2.5.10 estão satisfeitas,

$$
K(x, y)=\sum_{n=1}^{\infty} \lambda_{n}(\mathcal{K}) \phi_{n}(x) \overline{\phi_{n}(y)}
$$

onde a convergência é $L^{2}\left(\mathbb{R}^{m} \times \mathbb{R}^{m}\right)$, absoluta e uniforme, cada $\phi_{n}$ é autofunção de $\mathcal{K}$ associada ao autovalor $\lambda_{n}(\mathcal{K})$ e $\left\{\phi_{n}\right\}$ é uma família $L^{2}\left(\mathbb{R}^{m}\right)$-ortonormal. Ainda, $\phi_{n} \in$ $L^{1}\left(\mathbb{R}^{m}\right) \cap C_{o}\left(\mathbb{R}^{m}\right)$ quando $\lambda_{n}(\mathcal{K})$ é não-nulo. Além disso, pelo Teorema 2.5.6, $\mathcal{K}$ é nuclear $\mathrm{e}$

$$
\operatorname{tr}(\mathcal{K})=\int_{\mathbb{R}^{m}} K(x, x) d x=\sum_{n=1}^{\infty} \lambda_{n}(\mathcal{K})<\infty
$$

A equação acima implica que $\tilde{\mathcal{K}}$ é nuclear, uma vez que $\operatorname{tr}(\tilde{\mathcal{K}})=\operatorname{tr}(\mathcal{K})$ pela Proposição 2.6.8. Isso justifica parcialmente o item $(i i)$. Para finalizarmos a demonstração vamos mostrar que

$$
\operatorname{tr}(\tilde{\mathcal{K}})=\int_{\mathbb{R}^{m}} \tilde{K}(u, u) d u
$$

Para isso, considere a soma

$$
K_{p}(x, y):=\sum_{n=1}^{p} \lambda_{n}(\mathcal{K}) \phi_{n}(x) \overline{\phi_{n}(y)}, \quad x, y \in \mathbb{R}^{m} .
$$


Como $\lambda_{n}(\mathcal{K}) \geq 0, n=1,2, \ldots, p$, o Exemplo 2.1.2 implica que $K_{p} \in P D\left(\mathbb{R}^{m}\right)$. Logo, pelo Teorema 2.1.8,

$$
\left|K_{p}(x, y)\right| \leq K_{p}^{1 / 2}(x, x) K_{p}^{1 / 2}(y, y) \leq K^{1 / 2}(x, x) K^{1 / 2}(y, y), \quad x, y \in \mathbb{R}^{m} .
$$

Como $x \in \mathbb{R}^{m} \mapsto K^{1 / 2}(x, x)$ é um elemento de $L^{1}\left(\mathbb{R}^{m}\right)$ e $K \in L^{1}\left(\mathbb{R}^{m} \times \mathbb{R}^{m}\right)$, os teoremas de Fubini e da convergência dominada são aplicáveis. Logo,

$$
\int_{\mathbb{R}^{m}} \int_{\mathbb{R}^{m}} K(x, y) d x d y=\lim _{p \rightarrow \infty} \int_{\mathbb{R}^{m}} \int_{\mathbb{R}^{m}} K_{p}(x, y) d x d y
$$

e as integrais podem ser iteradas. O mesmo argumento se aplica ao núcleo

$$
K_{p}^{\prime}(x, y):=K_{p}(x, y) e^{-2 \pi i(x, y) \cdot(u, v)}, \quad x, y \in \mathbb{R}^{m},
$$

onde $(u, v)$ é um elemento de $\mathbb{R}^{m} \times \mathbb{R}^{m}$, ou seja,

$$
\int_{\mathbb{R}^{m}} \int_{\mathbb{R}^{m}}\left(\lim _{p \rightarrow \infty} K_{p}^{\prime}(x, y)\right) d x d y=\int_{\mathbb{R}^{m}} \int_{\mathbb{R}^{m}} K(x, y) e^{-2 \pi i(x, y) \cdot(u, v)} d x d y=\hat{K}(u, v) .
$$

Como

$$
\begin{aligned}
\int_{\mathbb{R}^{m}} \int_{\mathbb{R}^{m}} K_{p}^{\prime}(x, y) d x d y & =\sum_{n=1}^{p} \lambda_{n}(\mathcal{K}) \int_{\mathbb{R}^{m}} \phi_{n}(x) e^{-2 \pi i x \cdot u} d x \int_{\mathbb{R}^{m}} \overline{\phi_{n}(y)} e^{-2 \pi i y \cdot v} d y \\
& =\sum_{n=1}^{p} \lambda_{n}(\mathcal{K}) \hat{\phi_{n}}(u) \overline{\phi_{n}(v)} \\
& =\sum_{n=1}^{p} \lambda_{n}(\mathcal{K}) \hat{\phi}_{n}(u) \overline{\hat{\phi}_{n}(-v)}
\end{aligned}
$$

segue que

$$
\hat{K}(u, v)=\sum_{n=1}^{\infty} \lambda_{n}(\mathcal{K}) \hat{\phi}_{n}(u) \overline{\hat{\phi}_{n}(-v)}
$$

uma vez que $K \in L^{1}\left(\mathbb{R}^{m} \times \mathbb{R}^{m}\right)$ e $\phi_{n} \in L^{1}\left(\mathbb{R}^{m}\right)$ quando $\lambda_{n}(\mathcal{K})$ é não-nulo. Em particular,

$$
\tilde{K}(u, v)=\sum_{n=1}^{\infty} \lambda_{n}(\mathcal{K}) \hat{\phi}_{n}(u) \overline{\hat{\phi}_{n}(v)}, \quad(u, v) \in \mathbb{R}^{m} \times \mathbb{R}^{m} .
$$

Aplicando o Lema de Riemann-Lebesgue concluímos que todas as funções do conjunto $\left\{\hat{\phi}_{n}: \lambda_{n}(\mathcal{K}) \neq 0\right\}$ são uniformemente contínuas e se anulam no infinito. Portanto, a seqüência $\left\{\tilde{K}_{p}(u, u)\right\}$, onde

$$
\tilde{K}_{p}(u, u)=\sum_{n=1}^{p} \lambda_{n}(\mathcal{K})\left|\hat{\phi}_{n}(u)\right|^{2}, \quad p=1,2, \ldots,
$$


é uma seqüência não-decrescente de funções positivas convergindo pontualmente para $\tilde{K}(v, v)$. Pelo Teorema da Convergência Monótona,

$$
\lim _{p \rightarrow \infty} \int_{\mathbb{R}^{m}} \tilde{K}_{p}(u, u) d v=\int_{\mathbb{R}^{m}} \tilde{K}(u, u) d u
$$

e, conseqüentemente,

$$
\lim _{p \rightarrow \infty}\left(\sum_{n=1}^{p} \lambda_{n}(\mathcal{K}) \int_{\mathbb{R}^{m}}\left|\hat{\phi}_{n}(v)\right|^{2} d v\right)=\sum_{n=1}^{\infty} \lambda_{n}(\mathcal{K})=\int_{\mathbb{R}^{m}} \tilde{K}(u, u) d u .
$$

Segue que a função $u \in \mathbb{R}^{m} \mapsto \tilde{K}(u, u)$ é um elemento de $L^{1}\left(\mathbb{R}^{m}\right)$, pois $\tilde{K}(u, u) \geq 0$, $u \in \mathbb{R}^{m}$. Agora, aplicando o Teorema 2.5.5 a $\tilde{K}$, as demais afirmações seguem.

Observação 2.6.10. A referência [4] trata de resultado similar ao demonstrado acima no caso $m=1$. Lá, os autores afirmam que o teorema anterior ainda vale quando substituimos a condição de integrabilidade da função $x \in \mathbb{R}^{m} \mapsto K^{1 / 2}(x, x)$ pela seguinte condição mais fraca: $K \in L^{1}\left(\mathbb{R}^{m} \times \mathbb{R}^{m}\right)$. Não conseguimos ratificar este fato neste contexto mais geral. Na verdade, na demonstração em [4], afirma-se que a convergência uniforme da série permite a aplicação da transformada de Fourier e a conseqüente conclusão de que a série das transformadas é a transformada da série, com convergência pontual, fato este não muito claro para nós.

O teorema abaixo apresenta uma versão mais fraca do teorema anterior.

Teorema 2.6.11. Seja $K \in \mathcal{A}_{o}\left(\mathbb{R}^{m}\right)$ tal que $K \in L^{1}\left(\mathbb{R}^{m} \times \mathbb{R}^{m}\right)$. Então, além das conclusões do Teorema 2.5.5, as seguintes afirmações valem:

(i) O núcleo $\tilde{K}$ é um elemento de $L^{2} P D\left(\mathbb{R}^{m}\right) \cap C_{o}\left(\mathbb{R}^{m} \times \mathbb{R}^{m}\right)$ possuindo uma expansão $L^{2}\left(\mathbb{R}^{m} \times \mathbb{R}^{m}\right)$-convergente na forma

$$
\tilde{K}=\sum_{n=1}^{\infty} \lambda_{n}(\mathcal{K}) \hat{\phi}_{n} \otimes \overline{\hat{\phi}_{n}}
$$

onde $\left\{\lambda_{n}(\mathcal{K})\right\}$ é seqüência de autovalores do operador integral $\mathcal{K}$. Cada $\hat{\phi}_{n}$ é a transformada de Fourier da autofunção $\phi_{n}$ associada ao autovalor $\lambda_{n}(\mathcal{K})$ de $\mathcal{K}$. Além disso, cada $\hat{\phi}_{n}$ é uma autofunção do operador $\tilde{\mathcal{K}}$, a família $\left\{\hat{\phi}_{n}\right\}$ é $L^{2}\left(\mathbb{R}^{m}\right)$-ortonormal e $\hat{\phi}_{n}, \phi_{n} \in C_{o}\left(\mathbb{R}^{m}\right)$, quando $\lambda_{n}(\mathcal{K})$ é não-nulo.

(ii) O operador $\tilde{\mathcal{K}}$ é nuclear e

$$
\operatorname{tr}(|\tilde{\mathcal{K}}|)=\operatorname{tr}(\tilde{\mathcal{K}})=\operatorname{tr}(\mathcal{K})=\sum_{n=1}^{\infty} \lambda_{n}(\mathcal{K})=\int_{\mathbb{R}^{m}} K(x, x) d x
$$


Demonstração: Como $K \in \mathcal{A}_{o}\left(\mathbb{R}^{m}\right)$, as condições do Teorema 2.5.5 estão satisfeitas. Em particular, $\phi_{n} \in C_{o}\left(\mathbb{R}^{m}\right)$, quando $\lambda_{n}(\mathcal{K})$ é não-nulo. Logo,

$$
\begin{aligned}
\left\|\lambda_{n}(\mathcal{K}) \phi_{n}\right\|_{L^{1}\left(\mathbb{R}^{m}\right)}=\left\|\mathcal{K}\left(\phi_{n}\right)\right\|_{L^{1}\left(\mathbb{R}^{m}\right)} & =\int_{\mathbb{R}^{m}}\left|\int_{\mathbb{R}^{m}} K(x, y) \phi_{n}(y) d y\right| d x \\
\leq & M_{n} \int_{\mathbb{R}^{m}} \int_{\mathbb{R}^{m}}|K(x, y)| d x d y=M_{n}\|K\|_{\left.L^{1}\left(\mathbb{R}^{m} \times \mathbb{R}^{m}\right)\right)}
\end{aligned}
$$

onde $M_{n}=\sup \left\{\left|\phi_{n}(y)\right|: y \in \mathbb{R}^{m}\right\}<\infty$. Pela Proposição 2.6.8 temos que

$$
\tilde{K}=\sum_{n=1}^{\infty} \lambda_{n}(\mathcal{K}) \hat{\phi}_{n} \otimes \overline{\hat{\phi}_{n}}
$$

com convergência em $L^{2}\left(\mathbb{R}^{m} \times \mathbb{R}^{m}\right)$, e que $\tilde{K} \in L^{2} P D\left(\mathbb{R}^{m}\right)$. O restante do item $(i)$ segue da aplicação do Lema de Riemann-Lebesgue às funções $K$ e $\phi_{n}$. Como

$$
\operatorname{tr}(|\tilde{\mathcal{K}}|)=\operatorname{tr}(\tilde{\mathcal{K}})=\sum_{n=1}^{\infty} \lambda_{n}(\mathcal{K})
$$

e o Teorema 2.5.6 garante que

$$
\operatorname{tr}(\mathcal{K})=\sum_{n=1}^{\infty} \lambda_{n}(\mathcal{K})=\int_{\mathbb{R}^{m}} K(x, x) d x<\infty,
$$

o item (ii) segue. 



$-\frac{5}{3}$

\section{Decaimento de autovalores}

\subsection{Introdução}

Neste capítulo obtemos informações sobre o decaimento dos autovalores do operador integral $\mathcal{K}$, quando $K \in L^{2} P D(D)$ satisfaz alguma condição de suavidade e $D$ é um subconjunto fechado de $\mathbb{R}^{m}$ com medida estritamente positiva.

No caso em que $K \in \mathcal{A}_{o}(D)$, o Teorema 2.5.5 já nos garante que

$$
K(x, y)=\sum_{n=1}^{\infty} \lambda_{n}(\mathcal{K}) \phi_{n}(x) \overline{\phi_{n}(y)}, \quad x, y \in D,
$$

com convergência absoluta e uniforme, sendo que $\left\{\phi_{n}\right\}$ é um conjunto $L^{2}(D)$-ortonormal de autofunções de $\mathcal{K}$ associado à seqüência não-crescente e não-negativa de autovalores $\left\{\lambda_{n}(\mathcal{K})\right\}$. O Teorema 2.5.6 revela que $\mathcal{K}$ é nuclear e que

$$
0 \leq \operatorname{tr}(\mathcal{K})=\sum_{n=1}^{\infty} \lambda_{n}(\mathcal{K})=\int_{D} K(x, x) d x<\infty .
$$

Com isto, o Corolário 1.2.10 nos dá uma primeira informação sobre o decaimento dos autovalores de $\mathcal{K}$, a saber, que $\lambda_{n}(\mathcal{K})=o\left(n^{-1}\right)$ quando $n \rightarrow \infty$. Os exemplos da Seção 3.7 mostram que esta é a melhor estimativa que podemos obter deste teorema.

Em alguns casos específicos, a literatura nos fornece algumas estimativas melhores do que a citada acima: veja por exemplo as referências $[9,10,15,29,30,31]$ para o caso $D=[a, b]$ e as referências $[20,21]$ para conjuntos compactos mais gerais. Recentemente, a referência [5] analisou generalizações dos resultados das referências acima, no caso 
em que $K \in A_{o}(D)$ é suficientemente suave e $D$ é um intervalo fechado de $\mathbb{R}$. Outros resultados recentes sobre estimativas para os autovalores podem ser encontrados em $[6,7]$.

Neste capítulo, generalizamos os resultados de [5] para o caso em que $D$ é um subconjunto fechado de $\mathbb{R}^{m},(m \geq 1)$, com medida estritamente positiva.

\subsection{Aproximação por operadores de posto finito}

Nesta seção discutimos alguns resultados específicos da teoria dos operadores compactos em espaços de Hilbert. Os resultados são, na medida do possível, adaptados ao contexto do trabalho.

Iniciamos com um pequeno refinamento do Teorema 1.5.4. Sabemos, pelo Teorema 1.5.2, que um operador compacto e auto-adjunto sobre um espaço de Hilbert $\mathcal{H}$ é representável na forma

$$
T(x)=\sum_{n=1}^{\infty} \lambda_{n}(T)\left\langle x, x_{n}\right\rangle_{\mathcal{H}} x_{n}, \quad x \in \mathcal{H},
$$

onde $\left\{x_{n}\right\}$ é uma base ortonormal de $\mathcal{H}$ e $\left|\lambda_{1}(T)\right| \geq\left|\lambda_{2}(T)\right| \geq \cdots \geq 0$. É fácil ver que $\|T\|=\left|\lambda_{1}(T)\right|$. Em particular, se

$$
T_{j}(x):=\sum_{n=1}^{j} \lambda_{n}(T)\left\langle x, x_{n}\right\rangle_{\mathcal{H}} x_{n}, \quad x \in \mathcal{H}
$$

então $\left\|T-T_{j}\right\|=\left|\lambda_{j+1}(T)\right|=s_{j+1}(T)$. Logo, pelo Teorema 1.5.4, temos que a melhor aproximação para $T$ na norma de $\mathcal{L}(\mathcal{H})$ por operadores de posto no máximo $j$ é o operador $T_{j}$.

O lema seguinte mostra que, se $T$ é também nuclear, então $T_{j}$ é a melhor aproximação para $T$ na norma do traço por operadores auto-adjuntos de posto menor ou igual a $j$.

Lema 3.2.1. Nas condições acima, se $R \in L(\mathcal{H})$ é auto-adjunto de posto inferior a $j+1$, então

$$
\left\|T-T_{j}\right\|_{t r}=\sum_{n=j+1}^{\infty}\left|\lambda_{n}(T)\right| \leq\|T-R\|_{t r}
$$

Demonstração: Pelo Teorema 1.5.2 temos que

$$
(T-R)(x)=\sum_{n=1}^{\infty} \lambda_{n}(T-R)\left\langle x, y_{n}\right\rangle_{\mathcal{H}} y_{n}, \quad x \in \mathcal{H}
$$


onde $\left\{y_{n}\right\}$ é uma base ortonormal de $\mathcal{H}$. Defina $A_{0}=R$ e

$$
A_{p}(x)=R(x)+\sum_{n=1}^{p} \lambda_{n}(T-R)\left\langle x, y_{n}\right\rangle_{\mathcal{H}} y_{n}, \quad x \in \mathcal{H}, \quad p=1,2, \ldots
$$

O operador $A_{p}$ tem posto não-superior a $j+p$ e

$$
\left(T-A_{p}\right)(x)=\sum_{n=p+1}^{\infty} \lambda_{n}(T-R)\left\langle x, y_{n}\right\rangle_{\mathcal{H}} y_{n}, \quad x \in \mathcal{H} .
$$

Portanto, dos comentários anteriores, segue que

$$
\left|\lambda_{p+1}(T-R)\right|=\left\|T-A_{p}\right\| \geq\left\|T-T_{j+p}\right\|=\left|\lambda_{j+p+1}(T)\right|, \quad p=0,1, \ldots
$$

conseqüentemente,

$$
\left\|T-T_{j}\right\|_{t r}=\sum_{n=j+1}^{\infty}\left|\lambda_{n}(T)\right| \leq \sum_{n=1}^{\infty}\left|\lambda_{n}(T-R)\right|=\|T-R\|_{t r},
$$

o que completa a prova.

\subsection{A raiz quadrada de um operador}

Utilizando um refinamento do Lema 3.2.1 (veja o Teorema 3.4.4), pretendemos obter, pelo menos em alguns casos, informações sobre a taxa de decaimento dos autovalores do operador integral $\mathcal{K}$, quando $K$ é no mínimo $L^{2}$-positivo definido. Para tanto, vamos utilizar um operador $R_{j}$ apropriado, que depende de $\sqrt{\mathcal{K}}$. Esta seção é então dedicada ao estudo de algumas propriedades de $\sqrt{\mathcal{K}}$.

Pelo Teorema 1.4.18, todo operador linear positivo $T$ sobre um espaço de Hilbert $\mathcal{H}$ possui uma única raiz quadrada positiva. Em geral, é muito trabalhoso encontrar uma expressão fechada para a raiz quadrada de um operador, como os resultados ([33, p.196] e [43, p.221]) indicam. Entretanto, se existir uma base ortonormal $\left\{x_{n}\right\}$ de $\mathcal{H}$ e $T$ for representável na forma

$$
T(x)=\sum_{n=1}^{\infty} \lambda_{n}(T)\left\langle x, x_{n}\right\rangle_{\mathcal{H}} x_{n}, \quad x \in \mathcal{H}
$$

com $\lambda_{1}(T) \geq \lambda_{2}(T) \geq \cdots \geq 0$, poucos cálculos revelam que o operador linear $\mathcal{S}$ dado por

$$
\mathcal{S}(x)=\sum_{n=1}^{\infty} \lambda_{n}(T)^{1 / 2}\left\langle x, x_{n}\right\rangle_{\mathcal{H}} x_{n}, \quad x \in \mathcal{H},
$$

é a raíz quadrada de $T$. Em geral, $\sqrt{T}$ pode não herdar as propriedades de $T$. Por exemplo, se $T$ é um operador nuclear não existe garantia de que a raíz quadrada de $T$ é nuclear. Comecemos nosso estudo com o seguinte lema. 
Lema 3.3.1. Seja $D$ um subconjunto fechado de $\mathbb{R}^{m}$ com medida estritamente positiva. Se $K \in \mathcal{A}_{o}(D)$ tem uma representação na forma

$$
K(x, y)=\sum_{n=1}^{\infty} \lambda_{n}(\mathcal{K}) \phi_{n}(x) \overline{\phi_{n}(y)}, \quad x, y \in D
$$

onde $\left\{\phi_{n}\right\}$ é uma base ortonormal de $L^{2}(D)$ e $\lambda_{1}(\mathcal{K}) \geq \lambda_{2}(\mathcal{K}) \geq \ldots \geq 0$, então $\sqrt{\mathcal{K}}$ é um operador integral com núcleo $S \in L^{2} P D(D)$ dado por

$$
S(x, y)=\sum_{n=1}^{\infty} \lambda_{n}(\mathcal{K})^{1 / 2} \phi_{n}(x) \overline{\phi_{n}(y)}, \quad x, y \in D .
$$

Demonstração: Devido ao Teorema 2.5.6, $\mathcal{K}$ é nuclear. Logo, o núcleo $S$ dado por

$$
S=\sum_{n=1}^{\infty} \lambda_{n}(\mathcal{K})^{1 / 2} \phi_{n} \otimes \overline{\phi_{n}}
$$

é um elemento de $L^{2} P D(D)$ e $\|S\|_{L^{2}(D \times D)}^{2}=\operatorname{tr}(\mathcal{K})$. Pelo Teorema 2.5.5, temos ainda que

$$
\sum_{n=1}^{\infty} \lambda_{n}(\mathcal{K})\left|\phi_{n}(x)\right|^{2}=K(x, x), \quad x \in D,
$$

com convergência absoluta e uniforme. conseqüentemente, para cada $x \in D, S(x, \cdot) \in$ $L^{2}(D)$ e $\|S(x, \cdot)\|_{L^{2}(D)}^{2}=K(x, x)$. Com isso podemos utilizar o Teorema 1.4.2 para concluir que

$$
\mathcal{S}(f)(x)=\int_{D} S(x, y) f(y) d y=\sum_{n=1}^{\infty} \lambda_{n}(\mathcal{K})^{1 / 2}\left\langle f, \phi_{n}\right\rangle \phi_{n}(x), \quad x \in D .
$$

A unicidade de $\sqrt{\mathcal{K}}$ e as observações anteriores implicam que $\mathcal{S}=\sqrt{\mathcal{K}}$.

Lema 3.3.2. Seja $D$ um subconjunto fechado de $\mathbb{R}^{m}$ com medida estritamente positiva. Se $K \in \mathcal{A}_{o}(D)$, então $\operatorname{Im} \sqrt{\mathcal{K}} \subset C_{o}(D)$.

Demonstração: Se $K \in \mathcal{A}_{o}(D)$, do lema anterior segue que $\sqrt{\mathcal{K}}$ é um operador integral com núcleo $S \in L^{2}(D \times D)$ dado por

$$
S(x, y)=\sum_{n=1}^{\infty} \lambda_{n}(\mathcal{K})^{1 / 2} \phi_{n}(x) \overline{\phi_{n}(y)}, \quad x, y \in D .
$$

Pelo Teorema 2.5.5 já temos que cada autofunção $\phi_{n}$ associada a um autovalor não-nulo é contínua. Ainda,

$$
\sqrt{\mathcal{K}}(f)(x)=\sum_{n=1}^{\infty} \lambda_{n}(\mathcal{K})^{1 / 2}\left\langle f, \phi_{n}\right\rangle \phi_{n}(x), \quad x \in D, \quad f \in L^{2}(D) .
$$


Para obtermos a continuidade de $\sqrt{\mathcal{K}}(f), f \in L^{2}(D)$, basta, segundo o Teorema 1.2.4, mostrarmos que a convergência da série acima é uniforme. No entanto, pelas desigualdades de Cauchy-Schwarz e de Bessel, temos que, para $1 \leq p \leq q$,

$$
\begin{aligned}
\left|\sum_{n=p}^{q} \lambda_{n}(\mathcal{K})^{1 / 2}\left\langle f, \phi_{n}\right\rangle \phi_{n}(x)\right|^{2} & \leq \sum_{n=p}^{q}\left|\lambda_{n}(\mathcal{K})^{1 / 2} \phi_{n}(x)\right|^{2} \sum_{n=p}^{q}\left|\left\langle f, \phi_{n}\right\rangle\right|^{2} \\
& \leq\|f\|_{L^{2}(D)}^{2} \sum_{n=p}^{q} \lambda_{n}(\mathcal{K})\left|\phi_{n}(x)\right|^{2}, \quad x \in D .
\end{aligned}
$$

Logo, como $K \in C_{o}(D \times D)$ e a série na igualdade

$$
\sum_{n=1}^{\infty} \lambda_{n}(\mathcal{K})\left|\phi_{n}(x)\right|^{2}=K(x, x), \quad x \in D,
$$

converge uniformemente, o resultado segue.

Lema 3.3.3. Seja $D$ um subconjunto fechado de $\mathbb{R}^{m}$ com medida estritamente positiva. Se $K \in \mathcal{A}_{o}(D)$ e $\sqrt{\mathcal{K}}$ é um operador integral com núcleo $S$, então

$$
\int_{D} S(x, u) \overline{S(x, v)} d x=K(v, u), \quad u, v \in D .
$$

Demonstração: Devido ao Lema 3.3.1,

$$
S:=\sum_{n=1}^{\infty} \lambda_{n}(\mathcal{K})^{1 / 2} \phi_{n} \otimes \overline{\phi_{n}}
$$

é um elemento de $L^{2}(D \times D)$. Se $y \in D$, defina

$$
S_{j y}:=\sum_{n=1}^{j} \lambda_{n}(\mathcal{K})^{1 / 2} \overline{\phi_{n}(y)} \phi_{n}, \quad j=1,2, \ldots
$$

Claramente, $S_{j y} \in L^{2}(D)$ e $S(\cdot, y) \in L^{2}(D), y \in D$. Além disso, como

$$
\left\|S(\cdot, y)-S_{j y}\right\|_{L^{2}}^{2}=\sum_{n=j+1}^{\infty} \lambda_{n}(\mathcal{K})\left|\phi_{n}(y)\right|^{2}, \quad y \in D
$$

e

$$
\sum_{n=1}^{\infty} \lambda_{n}(\mathcal{K})\left|\phi_{n}(y)\right|^{2}=K(y, y), \quad y \in D
$$

segue que $S_{j y} \rightarrow S(\cdot, y)$ em $L^{2}(D)$. O Teorema 1.4 .2 garante que

$$
\left\langle S_{j u}, S_{j v}\right\rangle \rightarrow\langle S(\cdot, u), S(\cdot, v)\rangle, \quad u, v \in D
$$


enquanto que

$$
\left\langle S_{j u}, S_{j v}\right\rangle=\sum_{n=1}^{j} \lambda_{n}(\mathcal{K}) \phi_{n}(v) \overline{\phi_{n}(u)} \rightarrow K(v, u), \quad u, v \in D
$$

devido ao Teorema 2.5.5. Assim,

$$
\langle S(\cdot, u), S(\cdot, v)\rangle=\int_{D} S(x, u) \overline{S(x, v)} d x=K(v, u), \quad u, v \in D,
$$

o que finaliza a prova.

\subsection{Operadores de posto finito}

O objetivo principal desta seção é introduzir um operator integral de posto finito cujo núcleo é construído a partir de uma decomposição de um subconjunto $D$ de $\mathbb{R}^{m}$ por cubos $m$-dimensionais. Tal operador é utilizado na construção de discretizações de um operador integral genérico sobre $D$, como explicado no final da seção. Fica nítido então que os resultados aqui incluídos são técnicos.

O lema abaixo é uma conseqüência dos resultados das seções anteriores.

Lema 3.4.1. Sejam D um subconjunto fechado de $\mathbb{R}^{m}$ com medida estritamente positiva, $K$ um elemento de $\mathcal{A}_{o}(D), \mathcal{S}=\sqrt{\mathcal{K}}$ e $T \in \mathcal{L}\left(L^{2}(D)\right)$ um operador positivo. Valem as seguintes afirmações:

(i) Se T tem posto finito, então o operador $\mathcal{S} T \mathcal{S}$ tem posto finito e seu núcleo é um elemento de $\mathcal{A}_{o}(D)$;

(ii) $S e 0 \leq T \leq I$, onde I é o operador identidade sobre $L^{2}(D)$, então $0 \leq \mathcal{S} T \mathcal{S} \leq \mathcal{K}$.

Demonstração: $(i)$ Se $T$ tem posto finito, o Lema 1.4.13 garante que $T$ é um operador compacto. Sua positividade implica que ele é auto-adjunto. Pelo Teorema de HilbertSchmidt, segue que $T$ é um operador integral com núcleo $R \in L^{2}(D \times D)$, dado por

$$
R=\sum_{n=1}^{N} \lambda_{n}(T) \psi_{n} \otimes \overline{\psi_{n}}
$$

onde $N$ é um inteiro positivo, $\lambda_{n}(T) \geq 0, n=1,2, \ldots, N$, e $\left\{\psi_{n}: n=1,2, \ldots, N\right\}$ é um subconjunto $L^{2}(D)$-ortonormal. É fácil ver que

$$
\mathcal{S} T \mathcal{S}(f)(x)=\int_{D} S(x, u) \int_{D} R(u, v) \int_{D} S(v, y) f(y) d y d v d u, \quad x \in D, \quad f \in L^{2}(D) .
$$

Seja

$$
R_{1}(x, y)=\int_{D} \int_{D} S(x, u) R(u, v) S(v, y) d u d v, \quad x, y \in X
$$


e note que

$$
\begin{aligned}
R_{1}(x, y) & =\sum_{n=1}^{N} \lambda_{n}(T) \int_{D} S(x, u) \psi_{n}(u) d u \int_{D} S(v, y) \overline{\psi_{n}(v)} d v \\
& =\sum_{n=1}^{N} \lambda_{n}(T) \int_{D} S(x, u) \psi_{n}(u) d u \overline{\int_{D} S(y, v) \psi_{n}(v) d v}
\end{aligned}
$$

e, em particular, o operador integral associado $\mathcal{R}_{1}$ tem posto $\leq N$ e é positivo. Pelo Lema 3.3.2 temos que $R_{1}$ é uma função contínua. Logo, $R_{1} \in L^{2} P D(D) \cap C(D \times D)$. Mostremos agora que $R_{1} \in \mathcal{A}_{o}(D)$. Utilizando a desigualdade de Cauchy-Schwarz temos que

$$
\begin{aligned}
0 \leq R_{1}(x, x) & =\sum_{n=1}^{N} \lambda_{n}(T)\left|\int_{D} S(x, u) \psi_{n}(u) d u\right|^{2} \\
& \leq \sum_{n=1}^{N} \lambda_{n}(T) \int_{D}|S(x, u)|^{2} d u\left\|\psi_{n}\right\|_{L^{2}(D)}^{2} \\
& =\sum_{n=1}^{N} \lambda_{n}(T) \int_{D}|S(x, u)|^{2} d u, \quad x \in D .
\end{aligned}
$$

Como

$$
\int_{D}|S(x, u)|^{2} d u=\int_{D} S(x, u) \overline{S(x, u)} d u, \quad x \in D
$$

temos, pelo Lema 3.3.3, que

$$
\int_{D}|S(x, u)|^{2} d u=K(x, x), \quad x \in D .
$$

Logo,

$$
0 \leq R_{1}(x, x) \leq K(x, x) \sum_{n=1}^{N} \lambda_{n}(T), \quad x \in D,
$$

e, portanto, $x \in D \mapsto R_{1}(x, x) \in L^{1}(D)$. Isto mostra que $R_{1} \in C_{o}(D \times D)$ e, por conseguinte, $R_{1} \in \mathcal{A}_{o}(D)$. Da equação (3.1) e do Teorema de Fubini segue que $\mathcal{R}_{1}=$ $\mathcal{S} T \mathcal{S}$.

(ii) Se $0 \leq T \leq I$, então

$$
0 \leq\langle T(f), f\rangle \leq\langle f, f\rangle, \quad f \in L^{2}(D) .
$$

Em particular,

$$
\begin{aligned}
0 \leq\langle\mathcal{S} T \mathcal{S}(f), f\rangle=\langle T \mathcal{S}(f), \mathcal{S}(f)\rangle & \leq\langle\mathcal{S}(f), \mathcal{S}(f)\rangle \\
& =\left\langle\mathcal{S}^{2}(f), f\right\rangle=\langle\mathcal{K}(f), f\rangle, \quad f \in L^{2}(D)
\end{aligned}
$$


ou seja, $0 \leq \mathcal{S} T \mathcal{S} \leq \mathcal{K}$.

O resto da seção refere-se ao núcleo que construímos abaixo. Em princípio, a construção pode ser feita para um subconjunto $X$ qualquer de $\mathbb{R}^{m}$, contanto que o mesmo possua medida de Lebesgue positiva.

Definição 3.4.2. Seja $X_{r}$ a interseção de um subconjunto não-vazio $X$ de $\mathbb{R}^{m}$ com o cubo m-dimensional $[-r / 2, r / 2]^{m}, r>0$. Assuma que a medida de Lebesgue de $X_{r}$ é positiva e que

$$
[-r / 2, r / 2]^{m}=\cup_{j=1}^{N^{m}} C_{j}^{r},
$$

onde $\left\{C_{j}^{r}: j=1,2, \ldots, N^{m}\right\}$ é uma família de cubos $m$-dimensionais de mesmo volume e satisfazendo $C_{j}^{o} \cap C_{k}^{o}=\emptyset, j \neq k$. Se $\Gamma=\left\{n \in\left\{1, \ldots, N^{m}\right\}:\left|X \cap C_{n}^{r}\right|>0\right\}$, o núcleo associado a $\Gamma$ é o núcleo $R^{|\Gamma|} \in L^{2}(X \times X)$ dado por

$$
R^{|\Gamma|}(x, y)=\sum_{n \in \Gamma} \frac{1}{\left|X \cap C_{n}^{r}\right|} \chi_{X \cap C_{n}^{r}} \otimes \chi_{X \cap C_{n}^{r}}(x, y), \quad x, y \in X .
$$

O lema a seguir, estabelece propriedades básicas do operador integral associado ao núcleo da definição anterior.

Lema 3.4.3. Se $\mathcal{R}^{|\Gamma|}$ é o operador integral com núcleo $R^{|\Gamma|}$, então $\mathcal{R}^{|\Gamma|}$ tem posto finito $e 0 \leq \mathcal{R}^{|\Gamma|} \leq I$.

Demonstração: Como o conjunto $\left\{\left|X \cap C_{n}^{r}\right|^{-1 / 2} \chi_{X \cap C_{n}^{r}}\right\}_{n \in \Gamma}$ é ortonormal, segue que

$$
\mathcal{R}^{|\Gamma|}(f)=\sum_{n \in \Gamma}\left\langle f, \frac{\chi_{X \cap C_{n}^{r}}}{\left|X \cap C_{n}^{r}\right|^{1 / 2}}\right\rangle \frac{\chi_{X \cap C_{n}^{r}}}{\left|X \cap C_{n}^{r}\right|^{1 / 2}}, \quad f \in L^{2}(X) .
$$

Logo, $\mathcal{R}^{|\Gamma|}$ tem posto finito e igual a $|\Gamma|$, é positivo e seus únicos autovalores são 0 e 1. Em particular, $\left\|\mathcal{R}^{|\Gamma|}\right\|=1$. Utilizando a desigualdade de Cauchy-Schwarz ainda obtemos

$$
\begin{aligned}
\left\langle\left(I-\mathcal{R}^{|\Gamma|}\right)(f), f\right\rangle & =\langle f, f\rangle-\left\langle\mathcal{R}^{|\Gamma|}(f), f\right\rangle \\
& =\langle f, f\rangle-\left|\left\langle\mathcal{R}^{|\Gamma|}(f), f\right\rangle\right| \\
& \geq\langle f, f\rangle-\left\|\mathcal{R}^{|\Gamma|}\right\|\langle f, f\rangle=0, \quad f \in L^{2}(X),
\end{aligned}
$$

e o lema esta provado.

Voltamos às nossas condições básicas e mostramos um refinamento do Lema 3.2.1.

Teorema 3.4.4. Sejam $D$ um subconjunto fechado de $\mathbb{R}^{m}$ com medida estritamente positiva, $K$ um elemento de $\mathcal{A}_{o}(D), \mathcal{S}=\sqrt{\mathcal{K}}$ e $R^{|\Gamma|}$ dado na Definição 3.4.2. Nestas 
condições vale:

$$
\begin{aligned}
& \sum_{|\Gamma|+1}^{\infty} \lambda_{n}(\mathcal{K}) \leq \operatorname{tr}(\mathcal{K})-\operatorname{tr}\left(\mathcal{S} \mathcal{R}^{|\Gamma|} \mathcal{S}\right) \\
&=\sum_{n \in \Gamma} \frac{1}{\mid D \cap C_{n}^{r \mid}} \int_{D \cap C_{n}^{r}} \int_{D \cap C_{n}^{r}}[K(u, u)-K(v, u)] d u d v \\
&=\sum_{n \in \Gamma} \frac{1}{\left|D \cap C_{n}^{r}\right|} \int_{D \cap C_{n}^{r}} \int_{D \cap C_{n}^{r}}\left[\frac{K(u, u)+K(v, v)}{2}-K(v, u)\right] d u d v \\
&+\int_{D \backslash D_{r}} K(u, u) d u
\end{aligned}
$$

Demonstração: Os lemas 3.4.1 e 3.4.3 mostram que o núcleo de $\mathcal{S} \mathcal{R}^{|\Gamma|} \mathcal{S}$ é um elemento de $\mathcal{A}_{o}(D)$ e $\mathcal{K}-\mathcal{S} \mathcal{R}^{|\Gamma|} \mathcal{S} \geq 0$. Em particular, $\mathcal{S} \mathcal{R}^{|\Gamma|} \mathcal{S}$, que tem posto menor ou igual a $\Gamma$, é também nuclear. Logo, pelo Teorema 2.5.6,

$$
\begin{aligned}
\left\|\mathcal{K}-\mathcal{S} \mathcal{R}^{|\Gamma|} \mathcal{S}\right\|_{t r} & =\operatorname{tr}(\mathcal{K})-\operatorname{tr}\left(\mathcal{S} \mathcal{R}^{|\Gamma|} \mathcal{S}\right) \\
& =\int_{D} K(x, x) d x-\int_{D}\left[\int_{D} \int_{D} S(x, u) R^{|\Gamma|}(u, v) S(v, x) d u d v\right] d x
\end{aligned}
$$

onde $S$ é o núcleo gerador de $\mathcal{S}$. No entanto, aplicando o Teorema de Fubini, o Lema 3.3.3 e a Definição 3.4.2, temos que a integral

$$
I:=\int_{D}\left[\int_{D} \int_{D} S(x, u) R^{|\Gamma|}(u, v) S(v, x) d u d v\right] d x
$$

pode ser re-escrita como

$$
\begin{aligned}
I & =\int_{D} \int_{D} R^{|\Gamma|}(u, v)\left[\int_{D} S(x, u) S(v, x) d x\right] d u d v \\
& =\int_{D} \int_{D} R^{|\Gamma|}(u, v) K(v, u) d u d v \\
& =\int_{D_{r}} \int_{D_{r}} R^{|\Gamma|}(u, v) K(v, u) d u d v .
\end{aligned}
$$

Como

$$
\int_{D_{r}} R^{|\Gamma|}(u, v) d u=\sum_{n \in \Gamma} \chi_{X \cap C_{n}^{r}}(v)
$$

e

$$
\int_{D_{r}} R^{|\Gamma|}(u, v) d v=\sum_{n \in \Gamma} \chi_{D \cap C_{n}^{r}}(u)
$$


concluímos que estas integrais são iguais a 1 quase sempre em $D_{r}$. Assim,

$$
\begin{aligned}
\int_{D_{r}} K(x, x) d x & =\int_{D_{r}}\left[\int_{D_{r}} R^{|\Gamma|}(u, v) d u\right] K(v, v) d v \\
& =\int_{D_{r}}\left[\int_{D_{r}} R^{|\Gamma|}(u, v) d v\right] K(u, u) d u
\end{aligned}
$$

e

$$
\begin{aligned}
\left\|\mathcal{K}-\mathcal{S} \mathcal{R}^{|\Gamma|} \mathcal{S}\right\|_{t r} & =\int_{D_{r}} \int_{D_{r}} R^{|\Gamma|}(u, v)[K(u, u)-K(v, u)] d u d v+\int_{D \backslash D_{r}} K(u, u) d u \\
& =\sum_{n \in \Gamma} \frac{1}{\mid D \cap C_{n}^{r \mid}} \int_{D \cap C_{n}^{r}} \int_{D \cap C_{n}^{r}}[K(u, u)-K(v, u)] d u d v \\
& +\int_{D \backslash D_{r}} K(u, u) d u,
\end{aligned}
$$

ou

$$
\begin{aligned}
\left\|\mathcal{K}-\mathcal{S} \mathcal{R}^{|\Gamma|} \mathcal{S}\right\|_{t r} & =\int_{D_{r}} \int_{D_{r}} R^{|\Gamma|}(u, v)\left[\frac{K(u, u)+K(v, v)}{2}-K(v, u)\right] d u d v \\
& +\int_{D \backslash D_{r}} K(u, u) d u \\
& \left.=\sum_{n \in \Gamma} \frac{1}{\left|D \cap C_{n}^{r}\right|} \int_{D \cap C_{n}^{r}} \int_{D \cap C_{n}^{r}} \frac{K(u, u)+K(v, v)}{2}-K(v, u)\right] d u d v \\
& +\int_{D \backslash D_{r}} K(u, u) d u .
\end{aligned}
$$

Aplicando o Lema 3.2.1 temos o resultado.

\subsection{Núcleos lipschitzianos}

Nesta seção utilizamos o Teorema 3.4.4 para buscar estimativas para a razão de decaimento dos autovalores de operadores integrais gerados por núcleos de $\mathcal{A}_{o}(D), D$ um subconjunto de $\mathbb{R}^{m}$ com medida estritamente positiva, que satisfazem, adicionalmente, alguma condição do tipo Lipschitz.

A condição de Lipschitz de que trata nosso trabalho é como a seguir.

Definição 3.5.1. Sejam $X$ um subconjunto de $\mathbb{R}^{m}, \alpha \in(0,1], s \geq 0$ e $V$ um espaço vetorial normado. Um núcleo $K: X \times X \rightarrow V$ pertence à classe Lip $^{\alpha, s}(X)$ quando existir $\delta>0, r_{o} \geq 1, M \geq 0$ e uma função localmente integrável $A: \mathbb{R}^{m} \rightarrow[0,+\infty]$ tal que

$$
\|K(x, x)-K(x, y)\|_{V} \leq A(x)|x-y|^{\alpha}, \quad x, y \in X, \quad|x-y| \leq \delta,
$$


$e$

$$
\int_{[-r / 2, r / 2]^{m}} A(x) d x \leq M r^{s}, \quad r \geq r_{o} .
$$

Escrevemos $K \in \operatorname{Lip}_{A}^{\alpha, s}(X)$ quando queremos enfatizar a função $A$.

A definição acima é menos exigente do que outras encontradas na literatura (veja $[9,20,21,22]$ por exemplo). De fato, a primeira desigualdade da definição aparece com mais freqüência na forma

$$
\left\|K(x, y)-K\left(x, y^{\prime}\right)\right\|_{V} \leq A(x)\left|y-y^{\prime}\right|^{\alpha}, \quad x, y, y^{\prime} \in X
$$

O primeiro resultado da seção refere-se a uma estimativa básica para os autovalores do operador integral quando o núcleo correspondente satisfaz a condição de Lipschitz. Lembramos que os autovalores de um operador integral com núcleo positivo definido estão sempre ordenados em ordem não-crescente.

Lema 3.5.2. Sejam $D$ um subconjunto fechado de $\mathbb{R}^{m}$ com medida estritamente positiva e $K \in \mathcal{A}_{o}(D) \cap \operatorname{Lip}^{\alpha, s}(D)$. Assuma que

$$
\int_{D \backslash D_{r}} K(x, x) d x \leq \frac{C}{r^{\beta-m}}, \quad r \geq r_{1},
$$

onde $\beta>m, C \geq 0$ e $r_{1} \geq 1$. Defina

$$
\gamma:=\alpha \frac{\beta-m}{\beta-m+s+\alpha} .
$$

Se $n$ é um inteiro arbitrariamente grande, então existe uma constante não-negativa $C_{1}$ tal que

$$
\sum_{j=k(n)+1}^{\infty} n^{\gamma} \lambda_{j}(\mathcal{K}) \leq C_{1}
$$

para algum $k(n) \in\left\{0,1, \ldots, n^{m}\right\}$.

Demonstração: Sejam $r_{o}, \delta, M$ e $A(x)$ como na definição anterior e defina $\mathcal{S}:=\sqrt{\mathcal{K}}$. Seja $r \geq 1$ tal que $D_{r}:=D \cap[-r / 2, r / 2]^{m}$ tenha medida positiva. Sem perda de generalidade, podemos assumir que $r \geq r_{o}$ e $r \geq r_{1}$. Para qualquer cobertura de $[-r / 2, r / 2]^{m}$, como descrito na Definição 3.4.2, temos que

$$
|u-v| \leq m^{1 / 2} \frac{r}{N}, \quad u, v \in D \cap C_{j}^{r}, \quad n=1,2, \ldots, N^{m}
$$

Utilizando o Teorema 3.4.4, precisamos estimar o valor de

$$
\sum_{n \in \Gamma} \frac{1}{\left|D \cap C_{n}^{r}\right|} \int_{D \cap C_{n}^{r}} \int_{D \cap C_{n}^{r}}[K(u, u)-K(v, u)] d u d v+\int_{D \backslash D_{r}} K(u, u) d u .
$$


Como $K \in \operatorname{Lip}_{A}^{\alpha, s}(D)$ podemos estimar

$$
I:=\sum_{n \in \Gamma} \frac{1}{\left|D \cap C_{n}^{r}\right|} \int_{D \cap C_{n}^{r}} \int_{D \cap C_{n}^{r}}[K(u, u)-K(v, u)] d u d v
$$

na forma

$$
\begin{aligned}
|I| & \leq \sum_{n \in \Gamma} \frac{1}{\left|D \cap C_{n}^{r}\right|} \int_{D \cap C_{n}^{r}} \int_{D \cap C_{n}^{r}}|K(u, u)-K(v, u)| d u d v \\
& \leq \sum_{n \in \Gamma} \frac{1}{\left|D \cap C_{n}^{r}\right|} \int_{D \cap C_{n}^{r}} \int_{D \cap C_{n}^{r}} A(u) m^{\alpha / 2}\left(\frac{r}{N}\right)^{\alpha} d u d v \\
& \leq m^{\alpha / 2}\left(\frac{r}{N}\right)^{\alpha} \int_{[-r / 2, r / 2]^{m}} A(u) d u \\
& \leq M m^{\alpha / 2} \frac{r^{\alpha+s}}{N^{\alpha}}
\end{aligned}
$$

contanto que $m^{1 / 2} r / N \leq \delta$. O restante da prova consiste em re-escrever a estimativa acima para uma escolha de $r$ que depende de $N$. Precisamente, vamos usar $r=r(N):=$ $N^{(\alpha-\gamma) /(\alpha+s)}$. Claramente, $\lim _{N \rightarrow \infty} r(N)=\infty$ e $\lim _{N \rightarrow \infty} r(N) / N=0$. Portanto, para $N$ arbitrariamente grande, esta escolha não interfere na dedução da estimativa anterior. Assim, como $\gamma=(\beta-m)(\alpha-\gamma)(\alpha+s)^{-1}$, majoramos o módulo equação (3.2) por

$$
M m^{\alpha / 2} \frac{1}{N^{\gamma}}+\frac{C}{N^{\gamma}}=\frac{M m^{\alpha / 2}+C}{N^{\gamma}},
$$

para $N$ arbitrariamente grande. Para concluir a prova vamos usar o Teorema 3.4.4. Para tanto, observe que no contexto presente, o operador $\mathcal{S} \mathcal{R}^{|\Gamma|} \mathcal{S}$ tem posto $\leq N^{m}$. Sendo assim, tomando por exemplo $k(N):=|\Gamma| \leq N^{m}$ e utilizando o Teorema 3.4.4, concluímos que

$$
\sum_{j=k(N)+1}^{\infty} \lambda_{j}(\mathcal{K}) \leq \frac{C_{1}}{N^{\gamma}}
$$

onde $C_{1}=M m^{\alpha / 2}+C$. A desigualdade do enunciado segue.

Lema 3.5.3. Sejam $D$ um subconjunto fechado de $\mathbb{R}^{m}$ com medida estritamente positiva e $K \in \mathcal{A}_{o}(D) \cap \operatorname{Lip}^{\alpha, s}(D)$. Se D é compacto ou o suporte de $K$ é compacto, então existe uma constante $C_{1} \geq 0$ e $k(n) \in\left\{0,1, \ldots, n^{m}\right\}$ tais que

$$
\sum_{j=k(n)+1}^{\infty} n^{\alpha} \lambda_{j}(\mathcal{K}) \leq C_{1}, \quad n \in \mathbb{N}
$$

Demonstração: Se um dos casos de compacidade ocorre, existe $r_{o}>1$ tal que

$$
\int_{D \backslash D_{r}} K(u, u) d u=0, \quad r>r_{o} .
$$


Repetindo os argumentos da prova do teorema anterior majoramos o módulo equação (3.2) por

$$
M m^{\alpha / 2} \frac{\left(r_{o}+1\right)^{\alpha+s}}{N^{\alpha}}, \quad N \geq N_{o}
$$

onde $N_{o}$ é suficientemente grande. Tomando $C_{1}:=\max \left\{M m^{\alpha / 2}\left(r_{o}+1\right)^{\alpha+s}, N_{o} \operatorname{tr}(\mathcal{K})\right\}$, o resultado segue.

Vamos enunciar agora um dos resultados principais deste capítulo.

Teorema 3.5.4. Sejam D um subconjunto fechado de $\mathbb{R}^{m}$ com medida estritamente positiva e $K$ um elemento de $\mathcal{A}_{o}(D) \cap \operatorname{Lip}^{\alpha, s}(D)$.

(i) $S e$

$$
\int_{D \backslash D_{r}} K(x, x) d x \leq \frac{C}{r^{\beta-m}}, \quad r>r_{o},
$$

onde $\beta>m, C \geq 0$ e $r_{o} \geq 1$, então

$$
\lambda_{n}(\mathcal{K})=O\left(n^{-1-\gamma / m}\right), \quad \gamma=\alpha \frac{\beta-m}{\beta-m+s+\alpha}
$$

(ii) Se para cada $\beta>m$ existem constantes $C=C(\beta), r_{o}=r_{o}(\beta)$ tais que

$$
\int_{D \backslash D_{r}} K(x, x) d x \leq \frac{C}{r^{\beta-m}}, \quad r>r_{o},
$$

então

$$
\lambda_{n}(\mathcal{K})=o\left(n^{-1-\theta / m}\right), \quad \theta \in[0, \alpha)
$$

(iii) Se D é compacto ou o suporte de K é compacto, então o item (ii) pode ser refinado, sendo

$$
\lambda_{n}(\mathcal{K})=O\left(n^{-1-\alpha / m}\right)
$$

Demonstração: O item $(i)$ segue diretamente do Lema 3.5.2, do Teorema 1.2.7 e do Corolário 1.2.9 enquanto que (iii) é consequência do Lema 3.5.3 e do Corolário 1.2.9. Assuma agora as condições adicionais de $(i i)$. De $(i)$ segue que $\lambda_{n}(\mathcal{K})=O\left(n^{-1-\theta / m}\right)$, $\theta \in[0, \alpha)$, uma vez que $\gamma$ varia continuamente de 0 a $\alpha$, quando $\beta$ varia de $m$ a $\infty$. Para finalizar, suponha que $\lambda_{n}(\mathcal{K}) \neq o\left(n^{-1-\gamma_{o} / m}\right)$ para algum $\gamma_{0} \in[0, \alpha)$. Então existe $C>0$ tal que

$$
\limsup _{n \rightarrow \infty}\left\{n^{-1-\gamma_{0} / m} \lambda_{n}(\mathcal{K})\right\} \geq C
$$

Logo, se $\theta \in\left(\gamma_{0}, \alpha\right)$, o conjunto $\left\{n^{-1-\theta / m} \lambda_{n}(\mathcal{K})\right\}$ não é limitado, uma contradição. 
Observação 3.5.5. A condição $K(x, x)=O\left(|x|^{-\beta}\right)$, para algum $\beta>m$ implica a condição do item $(i)$ do teorema. De fato, sua validade implica que

$$
\begin{aligned}
\int_{D \backslash D_{r}} K(x, x) d x & \leq C \int_{\mathbb{R}^{m} \backslash B[0, r / 2]}|x|^{-\beta} d x \\
& =C \frac{2 \pi^{m / 2}}{\Gamma(m / 2)} \int_{r / 2}^{\infty} t^{m-\beta-1} d t \\
& =C \frac{2^{\beta-m+1} \pi^{m / 2}}{\Gamma(m / 2)} \frac{1}{r^{\beta-m}},
\end{aligned}
$$

para alguma constante $C$, onde $\Gamma$ denota da função gama usual. Da mesma forma, a condição $K(x, x)=O\left(|x|^{-\beta}\right)$, para todo $\beta>m$ implica a condição do item (ii) do teorema.

\subsection{Núcleos diferenciáveis}

Nesta seção reanalisamos os resultados da seção anterior, assumindo adicionalmente a existência de alguma derivada de $K$. Para tanto, inicialmente o conjunto $D$ tem que ser um subconjunto de $\mathbb{R}^{m}$ fechado, convexo e de interior não-vazio, enquanto que as demais hipóteses sobre $K$ são mantidas. Como estamos lidando com derivadas parciais utilizamos as letras $u$ e $v$ para as variáveis usuais de $\mathbb{R}^{m}$, para evitar confusão.

Comecemos com uma observação e uma conseqüência do Teorema 3.5.4.

Observação 3.6.1. (i) A expressão $\partial^{p} G(u, v) / \partial y^{p}$ representa uma transformação plinear de $\left(\mathbb{R}^{m}\right)^{p}$ em $\mathbb{C}([24$, p.1, 48]), tal que

$$
\frac{\partial^{p} G(u, v)}{\partial y^{p}}(h, h, \ldots, h)=\sum_{\alpha_{1}+\alpha_{2}+\ldots+\alpha_{m}=p} \frac{\partial^{p} G(u, v)}{\partial y_{1}^{\alpha_{1}} \partial y_{2}^{\alpha_{2}} \ldots \partial y_{m}^{\alpha_{m}}} h_{1}^{\alpha_{1}} h_{2}^{\alpha_{2}} \ldots h_{p}^{\alpha_{p}}
$$

$\operatorname{com} h=\left(h_{1}, h_{2}, \ldots, h_{m}\right) \in \mathbb{R}^{m}, y=\left(y_{1}, y_{2}, \ldots, y_{m}\right)$ e $\alpha_{i} \in\{0,1, \ldots, p\}, i=1,2, \ldots, m$. Empregamos a notação

$$
\frac{\partial^{p} G(u, v)}{\partial y^{p}} h^{p}:=\frac{\partial^{p} G(u, v)}{\partial y^{p}}(h, h, \ldots, h) .
$$

(ii) A norma de uma transformação p-linear $T$ de $V^{p}$ em $\mathbb{C}$ é definida por

$\|T\|_{\mathcal{L}_{p}(V, \mathbb{C})}=\inf \left\{C:\left|T\left(v_{1}, v_{2}, \ldots, v_{p}\right)\right| \leq C\left\|v_{1}\right\|_{V}\left\|v_{2}\right\|_{V} \ldots\left\|v_{p}\right\|_{V},\left(v_{1}, v_{2}, \ldots, v_{p}\right) \in V^{p}\right\}$

No caso em que $V=\mathbb{R}^{m}$ podemos mostrar que $\|T\|_{\mathcal{L}_{p}\left(\mathbb{R}^{m}, \mathbb{C}\right)}<\infty$. No que segue vamos denotar $\|T\|:=\|T\|_{\mathcal{L}_{p}\left(\mathbb{R}^{m}, \mathbb{C}\right)}$. 
Teorema 3.6.2. Sejam D um subconjunto fechado, convexo e de interior não-vazio de $\mathbb{R}^{m}$ e $K \in \mathcal{A}_{o}(D)$. Assuma que $\partial K / \partial x$ existe e é limitada em $D^{o} \times D^{o}$. Então os resultados do Teorema 3.5.4 ainda valem com $\gamma:=(\beta-m)(\beta+1)^{-1}$.

Demonstração: Inicialmente, basta notar que, sendo $D \partial$-mensurável, ele tem medida estritamente positiva. Dai, usando a Desigualdade do Valor Médio ([25, p.264]) e a continuidade de $K$ concluímos que

$$
|K(x, x)-K(x, y)|=|K(x, x)-K(y, x)| \leq M|x-y|, \quad x, y \in D,
$$

ou seja, $K \in \operatorname{Lip}^{1, m}(D)$. O resto segue do Teorema 3.5.4.

Para obtermos um resultado mais refinado utilizamos a próxima definição.

Definição 3.6.3. Sejam E um subconjunto de $\mathbb{R}^{m}$ e $V$ um espaço vetorial normado. Dizemos que a função $G: E \times E \rightarrow V$ é uniformemente contínua na diagonal de $E \times E$ quando dado $\epsilon>0$ existe $\delta=\delta(\epsilon)>0$, tal que

$$
\|G(u, v)-G(u, u)\|_{V}<\epsilon, \quad u, v \in E, \quad|u-v|<\delta .
$$

Lema 3.6.4. Seja $E$ um subconjunto aberto e convexo de $\mathbb{R}^{m}$. Seja $G: E \times E \rightarrow \mathbb{R}$ um núcleo hermitiano tal que $\partial G / \partial x$ é uniformemente contínua na diagonal de $E \times E$. Dado $\epsilon>0$, existe $\delta>0$ tal que

$$
\left|\frac{G(u, u)+G(v, v)}{2}-G(v, u)\right|<\delta_{1} \epsilon, \quad u, v \in E, \quad|u-v| \leq \delta_{1}<\delta .
$$

Demonstração: Por hipótese, para cada $\epsilon>0$ existe $\delta=\delta(\epsilon)>0$ tal que

$$
\left\|\frac{\partial G}{\partial x}(u, v)-\frac{\partial G}{\partial x}(u, u)\right\|<\epsilon, \quad u, v \in E, \quad|u-v| \leq \delta_{1}<\delta .
$$

Como $G$ é hermitiano obtemos ([24, p.1])

$$
\begin{aligned}
\frac{\partial G}{\partial y}(u, v) h & =\lim _{t \rightarrow 0} \frac{G(u, v+t h)-G(u, v)}{t} \\
& =\lim _{t \rightarrow 0} \frac{G(v+t h, u)-G(v, u)}{t} \\
& =\frac{\partial G}{\partial x}(v, u) h, \quad h \in \mathbb{R}^{m}, \quad u, v \in E .
\end{aligned}
$$

Logo,

$$
\frac{\partial G}{\partial y}(u, v)=\frac{\partial G}{\partial x}(v, u), \quad u, v \in E .
$$

Fixados $u, v \in E$, definimos $c:=(u+v) / 2$ e $d:=(v-u) / 2$ e escolhemos $\epsilon_{u, v}>0$ tal que

$$
c \pm t d=\frac{1}{2}[(u+v) \pm t(v-u)] \in E, \quad t \in\left(-\epsilon_{u, v}, 1+\epsilon_{u, v}\right) .
$$


A função $f_{u, v}:\left(-\epsilon_{u, v}, 1+\epsilon_{u, v}\right) \rightarrow \mathbb{R}$ dada por

$f_{u, v}(t)=\frac{G(c-t d, c-t d)+G(c+t d, c+t d)}{2}-G(c+t d, c-t d), \quad t \in\left(-\epsilon_{u, v}, 1+\epsilon_{u, v}\right)$,

é diferenciável em $E$. Além disso, $f_{u, v}(0)=0$ e

$$
f_{u, v}(1)=\frac{1}{2}(G(u, u)+G(v, v))-G(v, u) .
$$

Logo, pela Desigualdade do Valor Médio,

$$
\left|f_{u, v}(1)-f_{u, v}(0)\right| \leq \sup _{t \in(0,1)}\left\|f_{u, v}^{\prime}(t)\right\| \leq \sup _{t \in[0,1]}\left\|f_{u, v}^{\prime}(t)\right\|=\left\|f_{u, v}^{\prime}\left(t_{o}\right)\right\|,
$$

para algum $t_{o} \in[0,1]$. Utilizando a Regra da Cadeia, obtemos

$$
\begin{aligned}
f_{u, v}^{\prime}\left(t_{o}\right) & =\left(\frac{\partial G}{\partial x}\left(c+t_{o} d, c+t_{o} d\right)-\frac{\partial G}{\partial x}\left(c+t_{o} d, c-t_{o} d\right)\right) d \\
& +\left(\frac{\partial G}{\partial x}\left(c-t_{o} d, c+t_{o} d\right)-\frac{\partial G}{\partial x}\left(c-t_{o} d, c-t_{o} d\right)\right) d
\end{aligned}
$$

Desta forma,

$$
\left|f_{u, v}(1)\right| \leq f_{u, v}^{\prime}\left(t_{o}\right)<2 \epsilon|d| \leq \delta_{1} \epsilon, \quad|u-v| \leq \delta_{1}<\delta,
$$

e o lema está demonstrado.

Lema 3.6.5. Seja $D$ um subconjunto fechado, convexo e de interior não-vazio de $\mathbb{R}^{m}$. Seja $K \in \mathcal{A}_{o}(D)$ tal que $\partial K / \partial x$ é uniformemente contínua na diagonal de $D^{o} \times D^{o}$. Assuma que

$$
\int_{D \backslash D_{r}} K(x, x) d x \leq \frac{C}{r^{\beta-m}}, \quad r>r_{o},
$$

onde $\beta>m, C \geq 0$ e $r_{o} \geq 1$. Se

$$
\gamma:=\frac{\beta-m}{\beta+1}
$$

e $n$ é arbitrariamente grande, então existe uma constante $C_{1} \geq 0$ tal que

$$
\sum_{j=k(n)+1}^{\infty} n^{\gamma} \lambda_{j}(\mathcal{K}) \leq C_{1}
$$

para algum $k(n) \in\left\{0,1, \ldots, n^{m}\right\}$.

Demonstração: A prova é uma adaptação da prova do Lema 3.5.2. Sejam $\mathcal{S}=\sqrt{\mathcal{K}}$ e $\epsilon>0$. Tomemos $\delta>0$ tal que

$$
\left\|\frac{\partial K}{\partial x}(u, v)-\frac{\partial K}{\partial x}(u, u)\right\|<\epsilon, \quad u, v \in D^{\circ}, \quad|u-v|<\delta .
$$


Fixado $r \geq 1$ suficientemente grande, é possível escolher $N_{0} \in \mathbb{N}$ tal que

$$
m^{1 / 2} \frac{r}{N}<\delta, \quad N=N_{0}, N_{0}+1, \ldots
$$

Do Teorema 3.4.4 segue que precisamos apenas estimar o valor de

$$
\begin{array}{r}
\sum_{n \in \Gamma} \frac{1}{\left|D \cap C_{n}^{r}\right|} \int_{D \cap C_{n}^{r}} \int_{D \cap C_{n}^{r}}\left[\frac{K(u, u)+K(v, v)}{2}\right. \\
+\int_{D \backslash D_{r}} K(u, u) d u .
\end{array}
$$

Utilizando o Lema 2.4.1, podemos supor que a núcleo $K$ é uma função real quando estivermos interessados no cálculo de integrais como a anterior. Com isso, o lema anterior e a continuidade de $K$ implicam que

$$
\left|\frac{K(u, u)+K(v, v)}{2}-K(v, u)\right| \leq m^{1 / 2} \epsilon \frac{r}{N}, \quad u, v \in D \cap C_{n}^{r} .
$$

Notando que $\left|D \cap C_{n}^{r}\right| \leq(r / N)^{m}$, podemos majorar a soma

$$
S:=\sum_{j \in \Gamma} \frac{1}{\left|D \cap C_{n}^{r}\right|} \int_{D \cap C_{n}^{r}} \int_{D \cap C_{n}^{r}}\left[\frac{K(u, u)+K(v, v)}{2}-K(v, u)\right] d u d v
$$

por

$$
|S| \leq \sum_{j \in \Gamma} \frac{1}{\left|D \cap C_{n}^{r}\right|} \int_{D \cap C_{n}^{r}} \int_{D \cap C_{n}^{r}} m^{1 / 2} \epsilon \frac{r}{N} d u d v \leq m^{1 / 2} \epsilon \frac{r^{m+1}}{N} .
$$

No restante da prova utilizamos a majoração acima com escolhas específicas de $r$. Tomando $r=r(N):=N^{(1-\gamma) /(m+1)}$ vemos de imediato que $\lim _{N \rightarrow \infty} r(N)=\infty$ e $\lim _{N \rightarrow \infty} N^{-1} r(N)=0$. Ainda, $m^{1 / 2} N^{-1} r(N)<\delta$ e $r(N)>r_{o}$ para $N$ arbitrariamente grande. Utilizando as hipóteses e a estimativa anterior podemos majorar o módulo da equação (3.3) por

$$
m^{1 / 2} \epsilon \frac{r(N)^{m+1}}{N}+\frac{C}{r(N)^{\beta-m}},
$$

para $N$ grande. Como $(\beta-m)(1-\gamma)(m+1)^{-1}=\gamma$, segue do Teorema 3.4.4 que

$$
\sum_{j=k(N)+1}^{\infty} \lambda_{j}(\mathcal{K}) \leq \frac{C_{1}}{N^{\gamma}}
$$

onde $C_{1}=m^{1 / 2} \epsilon+C$ e $k(N):=|\Gamma| \leq N^{m}$. O lema está demonstrado.

Lema 3.6.6. Se no Lema 3.6.5, D é compacto ou o suporte de $K$ é compacto, então para cada $\epsilon>0$ existem $N=N(\epsilon)$ e $k(n) \in\left\{0,1, \ldots, n^{m}\right\}$ tais que

$$
\sum_{j=k(n)+1}^{\infty} n \lambda_{j}(\mathcal{K}) \leq \epsilon, \quad n \geq N
$$


Demonstração: Basta notar que nestes casos existe $r \geq 1$ tal que

$$
\int_{D \backslash D_{r}} K(u, u) d u=0
$$

Voltando à demonstração do Lema 3.6.5, dado $\epsilon_{1}=\epsilon m^{-1 / 2} r^{-m-1}>0$, existem $N=$ $N\left(m, \epsilon_{1}\right)$ e $k(n) \in\left\{0,1, \ldots, n^{m}\right\}$ tais que

$$
\sum_{j=k(n)+1}^{\infty} \lambda_{j}(\mathcal{K}) \leq m^{1 / 2} \epsilon_{1} \frac{r^{m+1}}{n}, \quad n \geq N .
$$

Isto conclui a prova.

Teorema 3.6.7. Seja D um subconjunto fechado, convexo e de interior não-vazio de $\mathbb{R}^{m}$. Seja $K \in \mathcal{A}_{o}(D)$ tal que $\partial K / \partial x$ é uniformemente contínua na diagonal de $D^{o} \times D^{o}$. (i) $\mathrm{Se}$

$$
\int_{D \backslash D_{r}} K(x, x) d x \leq \frac{C}{r^{\beta-m}}, \quad r \geq r_{o},
$$

onde $\beta>m, C \geq 0$ e $r_{o} \geq 1$, então

$$
\lambda_{n}(\mathcal{K})=O\left(n^{-1-\gamma / m}\right), \quad \gamma:=\frac{\beta-m}{\beta+1}
$$

(ii) Se para cada $C \geq 0$, existe $r_{o}=r_{o}(C) \geq 1$ tal que

$$
\int_{D \backslash D_{r}} K(x, x) d x \leq \frac{C}{r^{\beta-m}}, \quad r \geq r_{o},
$$

onde $\beta>m$, então

$$
\lambda_{n}(\mathcal{K})=o\left(n^{-1-\gamma / m}\right)
$$

(iii) Se para cada $\beta>m$, existem $C=C(\beta) \geq 0$ e $r_{o}=r_{o}(\beta) \geq 1$ tais que

$$
\int_{D \backslash D_{r}} K(x, x) d x \leq \frac{C}{r^{\beta-m}}, \quad r \geq r_{o},
$$

então

$$
\lambda_{n}(\mathcal{K})=o\left(n^{-1-\theta}\right), \quad \theta \in[0,1 / m) ;
$$

(iv) Se $D$ é compacto ou o suporte de $K$ é compacto, então o item (iii) pode ser refinado, sendo

$$
\lambda_{n}(\mathcal{K})=o\left(n^{-1-1 / m}\right) .
$$

Demonstração: $(i)$ Segue do Lema 3.6.5 e do Corolário 1.2.9.

(ii) Pela demonstração do Lema 3.6.5 segue que, para cada $\epsilon>0$ existem $N(\epsilon)$ e $k(N) \in\left\{0,1, \ldots, N^{m}\right\}$ tais que

$$
\sum_{j=k(N)+1}^{\infty} N^{\gamma} \lambda_{j}(\mathcal{K}) \leq m^{1 / 2} \epsilon+\epsilon, \quad N \geq N(\epsilon) .
$$


Logo, o Corolário 1.2.11 conclui a prova.

(iii) Basta utilizar a técnica usada na prova do Teorema 3.5.4-(ii).

(iv) Segue do Lema 3.6.6 e do Corolário 1.2.11.

Observação 3.6.8. A condição do item (ii) está satisfeita quando $K(x, x)=o\left(|x|^{-\beta}\right)$, $\beta>m$, enquanto que a condição do item (iii) está satisfeita quando $K(x, x)=$ $O\left(|x|^{-\beta}\right)$, para todo $\beta$.

Lema 3.6.9. Seja $E$ um subconjunto aberto e convexo de $\mathbb{R}^{m}$. Seja $G: E \times E \rightarrow \mathbb{R}$ um núcleo hermitiano real tal que $\partial G / \partial x \in \operatorname{Lip}^{\alpha, s}(E)$. Então, existe uma constante $\delta>0$ e uma função A, como na definição 3.5.1, tais que

$$
\begin{aligned}
\left|\frac{G(u, u)+G(v, v)}{2}-G(v, u)\right| \leq & A\left(\frac{u+t_{o} u}{2}+\frac{v-t_{o} v}{2}\right)|u-v|^{\alpha+1} \\
& +A\left(\frac{u-t_{o} u}{2}+\frac{v+t_{o} v}{2}\right)|u-v|^{\alpha+1},
\end{aligned}
$$

para algum $t_{o}=t_{o}(u, v) \in[0,1]$, quando $u, v \in E$ e $|u-v|<\delta$.

Demonstração: Basta utilizar a hipótese e proceder como na demonstração do Lema 3.6.4 para obtermos

$$
\begin{aligned}
\left|\frac{G(u, u)+K(v, v)}{2}-G(v, u)\right| \leq & \left\|\frac{\partial G}{\partial x}\left(c+t_{o} d, c+t_{o} d\right)-\frac{\partial G}{\partial x}\left(c+t_{o} d, c-t_{o} d\right)\right\||d| \\
& +\left\|\frac{\partial G}{\partial x}\left(c-t_{o} d, c+t_{o} d\right)-\frac{\partial G}{\partial x}\left(c-t_{o} d, c-t_{o} d\right)\right\||d| \\
\leq & A\left(\frac{u+v}{2}+t_{o} \frac{u-v}{2}\right)|u-v|^{\alpha+1} \\
& +A\left(\frac{u+v}{2}-t_{o} \frac{u-v}{2}\right)|u-v|^{\alpha+1},
\end{aligned}
$$

onde $d=(v-u) / 2, t_{o}=t_{o}(u, v) \in[0,1]$ e $u, v \in E$, com $|u-v|<\delta$.

Lema 3.6.10. Seja $D$ um subconjunto fechado, convexo e de interior não-vazio de $\mathbb{R}^{m}$. Seja $K \in \mathcal{A}_{o}(D)$ tal que $\partial K / \partial x \in \operatorname{Lip}_{A}^{\alpha, s}\left(D^{o}\right)$, onde $A$ é uma função constante. Assuma que

$$
\int_{D \backslash D_{r}} K(x, x) d x \leq \frac{C}{r^{\beta-m}}, \quad r>r_{o},
$$

para algum $\beta>m, \operatorname{com} C \geq 0$ e $r_{o} \geq 1$. Se

$$
\gamma:=(1+\alpha) \frac{\beta-m}{\beta+1+\alpha}
$$


e $n$ é arbitrariamente grande, então existe uma constante $C_{1} \geq 0$ tal que

$$
\sum_{j=k(n)+1}^{\infty} n^{\gamma} \lambda_{j}(\mathcal{K}) \leq C_{1},
$$

para algum $k(n) \in\left\{0,1, \ldots, n^{m}\right\}$.

Demonstração: A prova é novamente uma adaptação da prova do Lema 3.6.5. Sejam $\mathcal{S}=\sqrt{\mathcal{K}}$ e tomemos $\delta>0$ como no lema anterior. Fixado $r \geq 1$ suficientemente grande, é possível escolher $N_{o} \in \mathbb{N}$ tal que

$$
m^{1 / 2} \frac{r}{N}<\delta, \quad N=N_{o}, N_{o}+1, \ldots
$$

Utilizando o Teorema 3.4.4 vamos estimar a seguinte integral

$$
\begin{array}{r}
\sum_{n \in \Gamma} \frac{1}{\left|D \cap C_{n}^{r}\right|} \int_{D \cap C_{n}^{r}} \int_{D \cap C_{n}^{r}}\left[\frac{K(u, u)+K(v, v)}{2}-K(v, u)\right] d u d v \\
+\int_{D \backslash D_{r}} K(u, u) d u .
\end{array}
$$

Pelo o Lema 2.4.1 podemos supor que a núcleo $K$ é uma função real quando estivermos interessados no cálculo de integrais como a anterior. Com isso, utilizando a continuidade de $K$, obtemos

$$
\left|\frac{K(u, u)+K(v, v)}{2}-K(v, u)\right| \leq 2 M m^{(\alpha+1) / 2}\left(\frac{r}{N}\right)^{\alpha+1}, \quad u, v \in D \cap C_{n}^{r} .
$$

Notando que $\left|D \cap C_{n}^{r}\right| \leq(r / N)^{m}$, o primeiro somando da equação (3.4) pode ser majorado em módulo por

$$
m^{(\alpha+1) / 2} 2 M \frac{r^{\alpha+1+m}}{N^{\alpha+1}} .
$$

Definindo $r=r(N):=N^{\theta}$, onde $\theta:=(1+\alpha)(\beta+1+\alpha)^{-1}$, é claro que $r(N) \rightarrow \infty$ e $r(N) / N \rightarrow 0$, quando $N \rightarrow \infty$. Ainda, existe $N_{m} \in \mathbb{N}$ tal que, $m^{1 / 2} r(N) / N<\delta$ e $r(N)>r_{o}$, para $N \geq N_{m}$. Utilizando as condições do enunciado e a estimativa anterior, majoramos o módulo da equação (3.4) por

$$
m^{(1+\alpha) / 2} 2 M \frac{r(N)^{1+\alpha+m}}{N^{1+\alpha}}+\frac{C}{r(N)^{\beta-m}}, \quad N \geq N_{m}
$$

Como $\theta(\beta-m)=\gamma$, segue do Teorema 3.4.4 que

$$
\sum_{j=k(N)+1}^{\infty} \lambda_{j}(\mathcal{K}) \leq \frac{C_{1}}{N^{\gamma}}, \quad N \geq N_{m},
$$

onde $C_{1}=m^{(1+\alpha) / 2} 2 M+C$ e $k(N):=|\Gamma| \leq N^{m}$. O lema está demonstrado. 
Lema 3.6.11. Seja D como no Lema 3.6.10. Se D é compacto ou o suporte de $K$ é compacto, então existem $N, C$ e $k(n) \in\left\{0,1, \ldots, n^{m}\right\}$ tais que

$$
\sum_{j=k(n)+1}^{\infty} n^{1+\alpha} \lambda_{j}(\mathcal{K}) \leq C, \quad n \geq N .
$$

Demonstração: Basta notar que em ambos os casos existe $r \geq 1$ tal que

$$
\int_{D \backslash D_{r}} K(u, u) d u=0
$$

Logo, o resultado segue da demonstração do Lema 3.6.10.

Teorema 3.6.12. Sejam D um subconjunto fechado, convexo e de interior não-vazio de $\mathbb{R}^{m}$ e $K \in \mathcal{A}_{o}(D)$ tal que $\partial K / \partial x \in \operatorname{Lip}_{A}^{\alpha, m}\left(D^{o}\right)$, onde A é uma função constante. (i) $S e$

$$
\int_{D \backslash D_{r}} K(x, x) d x \leq \frac{C}{r^{\beta-m}}, \quad r \geq r_{o},
$$

para algum $\beta>m$, com $C \geq 0$ e $r_{o} \geq 1$, então

$$
\lambda_{n}(\mathcal{K})=O\left(n^{-1-\gamma / m}\right), \quad \gamma:=(1+\alpha) \frac{\beta-m}{\beta+1+\alpha} .
$$

(ii) Se para cada $\beta>m$, existe $C=C(\beta) \geq 0$ e $r_{o}=r_{o}(\beta) \geq 1$ tais que

$$
\int_{D \backslash D_{r}} K(x, x) d x \leq \frac{C}{r^{\beta-m}}, \quad r \geq r_{o},
$$

então

$$
\lambda_{n}(\mathcal{K})=o\left(n^{-1-\theta}\right), \quad \theta \in[0,(1+\alpha) / m) ;
$$

(iii) Se D é compacto ou o suporte de K é compacto, então o item (ii) pode ser refinado, sendo

$$
\lambda_{n}(\mathcal{K})=O\left(n^{-1-(1+\alpha) / m}\right)
$$

Demonstração: O item $(i)$ segue do Lema 3.6.10 e do Corolário 1.2.9. Em (ii) basta utilizar a técnica usada na prova do Teorema 3.5.4-(ii). Quanto a (iii), ele segue do Lema 3.6.11 e do Corolário 1.2.9.

O teorema seguinte é um corolário do teorema anterior.

Teorema 3.6.13. Sejam D um subconjunto fechado, convexo e de interior não-vazio de $\mathbb{R}^{m}$ e $K \in \mathcal{A}_{o}(D)$. Assuma que $\partial K / \partial x$ é contínua e que a derivada mista $\partial^{2} K / \partial x \partial y$ existe e é limitada em $D^{\circ} \times D^{o}$. Então os resultados do Teorema 3.6.12 ainda valem com $\gamma:=2(\beta-m)(\beta+2)^{-1}$. 
Demonstração: Basta usar a Desigualdade do Valor Médio e a continuidade de $\partial K / \partial x$ para concluir que

$$
\left\|\frac{\partial K(u, v)}{\partial x}-\frac{\partial K(u, u)}{\partial x}\right\| \leq M|u-v|, \quad u, v \in D^{\circ},
$$

ou seja, $\partial K / \partial x \in \operatorname{Lip}_{A}^{1, m}\left(D^{o}\right)$, onde $A$ é uma função constante. O resto segue do Teorema 3.6.12.

Observação 3.6.14. Note pequenas adaptações nas demonstrações e construções feitas neste capítulo nos possibilitam provar os resultados desta seção quando o conjunto $D$ for fechado e igual a união finita de convexos de medida estritamente positiva. Ainda, utilizando o Teorema 2.4.7 e alguns resultados de análise na esfera podemos provar resultados análogos aos deste capítulo para certos subconjuntos de $S^{m}$.

Utilizando os resultados desta seção e os resultados dos artigos [6, 7, 21] acreditamos que as seguintes conjecturas sejam verdadeiras.

Conjectura 3.6.15. Sejam D um subconjunto de $\mathbb{R}^{m}$ igual a união finita de convexos fechados e de interior não-vazio e p um inteiro não negativo. Seja $K \in \mathcal{A}_{o}(D)$ tal que $\partial^{p} K / \partial^{p} x$ é uniformemente contínua na diagonal de $D^{o} \times D^{o}$.

(i) $\mathrm{Se}$

$$
\int_{D \backslash D_{r}} K(x, x) d x \leq \frac{C}{r^{\beta-m}}, \quad r \geq r_{o},
$$

onde $\beta>m, C \geq 0$ e $r_{o} \geq 1$, então

$$
\lambda_{n}(\mathcal{K})=O\left(n^{-1-\gamma / m}\right), \quad \gamma:=p \frac{\beta-m}{\beta+p} ;
$$

(ii) Se para cada $C \geq 0$, existe $r_{o}=r_{o}(C) \geq 1$ tal que

$$
\int_{D \backslash D_{r}} K(x, x) d x \leq \frac{C}{r^{\beta-m}}, \quad r \geq r_{o},
$$

onde $\beta>m$, então

$$
\lambda_{n}(\mathcal{K})=o\left(n^{-1-\gamma / m}\right)
$$

(iii) Se para cada $\beta>m$, existe $C=C(\beta) \geq 0$ e $r_{o}=r_{o}(\beta) \geq 1$ tais que

$$
\int_{D \backslash D_{r}} K(x, x) d x \leq \frac{C}{r^{\beta-m}}, \quad r \geq r_{o},
$$

então

$$
\lambda_{n}(\mathcal{K})=o\left(n^{-1-\theta}\right), \quad \theta \in[0, p / m) ;
$$

(iv) Se D é compacto ou o suporte de $K$ é compacto, então

$$
\lambda_{n}(\mathcal{K})=o\left(n^{-1-p / m}\right) .
$$


De fato, o caso $p=0$ é imediato, o caso $m=1$ ( $p$ qualquer) já esta provado em [6] enquanto que o caso $p=1$ ( $m$ qualquer) já foi considerado no Teorema 3.6.7.

Conjectura 3.6.16. Sejam $D$ um subconjunto de $\mathbb{R}^{m}$ igual a união finita de convexos fechados e de interior não-vazio, $p$ um inteiro não negativo e $K \in \mathcal{A}_{o}(D)$ tal que $\partial^{p} K / \partial^{p} x \in \operatorname{Lip}^{\alpha, s}\left(D^{o}\right)$.

(i) $\mathrm{Se}$

$$
\int_{D \backslash D_{r}} K(x, x) d x \leq \frac{C}{r^{\beta-m}}, \quad r \geq r_{o},
$$

para algum $\beta>m$, com $C \geq 0$ e $r_{o} \geq 1$, então

$$
\lambda_{n}(\mathcal{K})=O\left(n^{-1-\gamma / m}\right), \quad \gamma:=(p+\alpha) \frac{\beta-m}{\beta-m+p+\alpha+s} ;
$$

(ii) Se para cada $\beta>m$, existem $C=C(\beta) \geq 0$ e $r_{o}=r_{o}(\beta) \geq 1$ tais que

$$
\int_{D \backslash D_{r}} K(x, x) d x \leq \frac{C}{r^{\beta-m}}, \quad r \geq r_{o},
$$

então

$$
\lambda_{n}(\mathcal{K})=o\left(n^{-1-\theta}\right), \quad \theta \in[0,(p+\alpha) / m) ;
$$

(iii) Se D é compacto ou o suporte de $K$ é compacto, então

$$
\lambda_{n}(\mathcal{K})=O\left(n^{-1-(p+\alpha) / m}\right) .
$$

Se $\partial^{p} K / \partial x^{p} \in \operatorname{Lip}_{A}^{\alpha, m}\left(D^{o}\right), A$ constante, a conjectura acima já foi ratificada nos seguintes casos: $m=1([7])$ e $p=1$ (Teorema 3.6.12). Por outro lado, o caso $p=0$ e $\partial^{p} K / \partial x^{p} \in \operatorname{Lip}^{\alpha, s}(D)$ segue do Teorema 3.5.4.

\subsection{Análise das estimativas}

Os exemplos a seguir indicam que as estimativas obtidas no Teorema 3.6.7 e as mencionadas na Conjectura 3.6.15 são ótimas, pelo menos no caso em que o domínio $D \times D$ é compacto. De fato, dado $\epsilon>0$, existem núcleos satisfazendo as condições do teorema e da conjectura, de modo que os autovalores $\lambda_{n}$ dos operadores integrais correspondentes são tais que $\lambda_{n} \neq o\left(n^{-1-p / m-\epsilon}\right)$. Isto sugere que os resultados também são ótimos quando $D$ é não-compacto. Os exemplos foram obtidos ou adaptados de $[21,31,32]$.

Exemplo 3.7.1. Sejam $p$ um inteiro não negativo, $\epsilon>0$ e $K \in L^{2} P D([-1,1])$ dado por

$$
K(x, y)=\sum_{n=1}^{\infty} \frac{1}{n^{p+1+\epsilon}} \cos (n \pi x) \cos (n \pi y), \quad x, y \in[-1,1]
$$


É fácil ver que a seqüência $\left\{\phi_{n}\right\}$, onde $\phi_{n}(x):=\cos (n \pi x), x \in[-1,1]$, é $L^{2}([-1,1])$ ortonormal e que $\lambda_{n}(\mathcal{K})=n^{-1-p-\epsilon}, n=1,2, \ldots$ Logo, $\lambda_{n}(\mathcal{K})=O\left(n^{-1-p-\epsilon}\right)$ e $\lambda_{n}(\mathcal{K}) \neq$ $o\left(n^{-1-p-\epsilon}\right)$. Como a série

$$
\sum_{n=1}^{\infty} \frac{n^{q}}{n^{p+1+\epsilon}}, \quad 0 \leq q \leq p
$$

é convergente, temos que as derivadas parciais de ordem menor ou igual a $p$ de $K$ são contínuas ([23, p.249,270]). Em particular, temos que $K \in C^{p}([-1,1] \times[-1,1])$.

Exemplo 3.7.2. Sejam $\alpha:=p+2+\epsilon, p$ um inteiro não-negativo, $\epsilon>0$ e $b_{m n}$ a $m, n$-ésima entrada da matriz

$$
\left(\begin{array}{cccccc}
1 & 2^{-\alpha} & 3^{-\alpha} & \cdots & n^{-\alpha} & \cdots \\
2^{-\alpha} & 3^{-\alpha} & & & & \\
3^{-\alpha} & & & & & \\
\vdots & & & & & \\
n^{-\alpha} & & & & \\
\vdots & & & & &
\end{array}\right)
$$

Observando a matriz deduzimos que

$$
\sum_{m=1}^{\infty} \sum_{n=1}^{\infty} b_{m n}^{2}=\sum_{m=1}^{\infty} \sum_{n=1}^{m} \frac{1}{m^{2 \alpha}}=\sum_{m=1}^{\infty} \frac{1}{m^{2 \alpha-1}}<\infty .
$$

Logo, o núcleo $K$ dado por

$$
K(x, y)=\sum_{m=1}^{\infty} \sum_{n=1}^{\infty} b_{m n} e^{2 \pi i\left(m x_{1}+n x_{2}\right)} e^{-2 \pi i\left(m y_{1}+n y_{2}\right)}, \quad x, y \in[0,1]^{2}
$$

é um elemento de $L^{2} P D\left([0,1]^{2}\right)$. Utilizando a continuidade do produto interno, temos que a seqüência dupla $\left\{\phi_{m n}\right\}$, onde $\phi_{m n}\left(x_{1}, x_{2}\right):=e^{2 \pi i\left(m x_{1}+n x_{2}\right)}, m, n=1,2, \ldots$, é $L^{2}\left([0,1]^{2}\right)$-ortonormal e que $\phi_{m n}$ é autofunção de $\mathcal{K}$. Observando novamente a matriz e tomando $j, l \in \mathbb{N}$ tais que $j+l \leq p$, temos que

$$
\sum_{m=1}^{\infty} \sum_{n=1}^{\infty} m^{j} n^{l} b_{m n} \leq \sum_{m=1}^{\infty} \sum_{n=1}^{m} \frac{m^{l+j}}{m^{\alpha}}=\sum_{m=1}^{\infty} \frac{m^{l+j+1}}{m^{\alpha}}<\infty .
$$

Isto implica que $\partial^{j+l} K / \partial x^{j} \partial y^{l}$ é contínua e que $K \in C^{p}\left([0,1]^{2} \times[0,1]^{2}\right)$. Além disso, ordenando os autovalores de $\mathcal{K}$ em ordem não-crescente de módulo, temos que $\lambda_{n(n+1) / 2}(\mathcal{K})=$ $n^{-\alpha}$ e, portanto, $\lambda_{n}(\mathcal{K})=O\left(n^{-\alpha / 2}\right)$. Como

$$
\left(\frac{n(n+1)}{2}\right)^{\alpha / 2} \lambda_{n(n+1) / 2}(\mathcal{K})=\left(\frac{n(n+1)}{2}\right)^{\alpha / 2} n^{-\alpha} \geq \frac{1}{2^{\alpha / 2}} \neq 0
$$

podemos concluir que $\lambda_{n}(\mathcal{K}) \neq o\left(n^{-\alpha / 2}\right)$. 
Exemplo 3.7.3. Sejam $D=[0,2 \pi]^{m}, p$ um inteiro não-negativo e $\epsilon \in(0,1)$. Seja $n=\left(n_{1}, n_{2}, \ldots, n_{m}\right) \in \mathbb{N}^{m}$ e defina $\phi_{n}(x):=e^{i x \cdot n}, x \in D$. É fácil verificar que $\left\{\phi_{n}\right\}$ é $L^{2}(D)$-ortonormal. Para cada $k \in \mathbb{N}$, sejam

$$
\begin{gathered}
b_{k}:=2^{-k(m+p+\epsilon)} \\
I_{k}=\left\{n \in \mathbb{N}^{m}: 2^{k} \leq n_{j}<2^{k+1}, j=1,2, \ldots, m\right\}
\end{gathered}
$$

e

$$
K(x, y)=\sum_{k=1}^{\infty} b_{k} \sum_{n \in I_{k}} \phi_{n}(x) \overline{\phi_{n}(y)}, \quad x, y \in D .
$$

Como a cardinalidade de $I_{k}$ é $2^{k m} \mathrm{e}$

$$
\sum_{k=1}^{\infty} b_{k} 2^{k m}=\sum_{k=1}^{\infty} \frac{1}{2^{k(m+p+\epsilon)}} 2^{k m}=\sum_{k=1}^{\infty} \frac{1}{2^{k(p+\epsilon)}}<\infty,
$$

temos que $K$ é contínua, em particular, $K \in L^{2} P D(D)$. Pela continuidade do produto interno, temos que $\phi_{n}, n \in I_{k}$, é autofunção de $\mathcal{K}$, associada ao autovalor $b_{k}$, que tem multiplicidade $2^{k m}$. Além disso, se $\alpha=\left(\alpha_{1}, \alpha_{2}, \ldots, \alpha_{m}\right) \in \mathbb{N}^{m}$ é tal que $\sum_{l=1}^{m} \alpha_{l} \leq p$ e $n=\left(n_{1}, n_{2}, \ldots, n_{m}\right) \in I_{k}$, então

$$
\prod_{l=1}^{m} n_{l}^{\alpha_{l}} \leq 2^{(k+1) p}
$$

Logo, $K \in C^{p}(D \times D)$, uma vez que

$$
\sum_{k=1}^{\infty} b_{k} 2^{k m} 2^{(k+1) p}=\sum_{k=1}^{\infty} \frac{1}{2^{k(m+p+\epsilon)}} 2^{k m+k p+p}=2^{p} \sum_{k=1}^{\infty} \frac{1}{2^{k \epsilon}}<\infty,
$$

e, portanto, as derivadas parciais

$$
\frac{\partial^{j+l} K}{\partial x^{j} \partial y^{l}}, \quad l+j \leq p
$$

são contínuas. Se ordenarmos os autovalores de $\mathcal{K}$ em ordem não-crescente e tomarmos

$$
q=\sum_{j=1}^{k} 2^{m j}=2^{m} \frac{1-2^{m k}}{1-2^{m}}
$$

então $\lambda_{q}(\mathcal{K})=2^{-k(m+p+\epsilon)}$. Segue que

$$
\left(\frac{2^{m(k+1)}}{2^{m}-1}\right)^{1+(p+\epsilon) / m} 2^{-k(m+p+\epsilon)} \leq q^{1+(p+\epsilon) / m} \lambda_{q}(\mathcal{K}) \leq\left(\frac{2^{m(k+2)}}{2^{m}-1}\right)^{1+(p+\epsilon) / m} 2^{-k(m+p+\epsilon)}
$$

Da equação acima segue que $\lambda_{j}(\mathcal{K})=O\left(j^{-1-(p+\epsilon) / m}\right)$ e que $\lambda_{j}(\mathcal{K}) \neq o\left(j^{-1-(p+\epsilon) / m}\right)$. 


\subsection{Considerações finais}

Para finalizar este trabalho enunciamos um resultado que resume, de uma certa forma, os resultados obtidos neste trabalho para o caso em que $D=\mathbb{R}^{m}$, mais especificamente, generalizamos os resultados obtidos nos artigos [4, 5].

Corolário 3.8.1. Seja $K \in \mathcal{A}_{o}\left(\mathbb{R}^{m}\right)$ tal que $K(x, x)=O\left(|x|^{-\beta}\right), \beta>2 m$. Neste caso as seguintes afirmações são verdadeiras:

(i) O núcleo $K$ é da forma

$$
K(x, y)=\sum_{1}^{\infty} \lambda_{n}(\mathcal{K}) \phi_{n}(x) \overline{\phi_{n}(y)}, \quad x, y \in \mathbb{R}^{m},
$$

com convergência $L^{2}\left(\mathbb{R}^{m} \times \mathbb{R}^{m}\right)$, absoluta e uniforme, sendo $\left\{\phi_{n}\right\}$ uma base ortonormal de $L^{2}\left(\mathbb{R}^{m}\right)$ formada por autofunções associadas aos autovalores $\left\{\lambda_{n}(\mathcal{K})\right\}$ do operador $\mathcal{K}$, satisfazendo $\lambda_{1}(\mathcal{K}) \geq \lambda_{2}(\mathcal{K}) \geq \cdots \geq 0$. Além disso $\mathcal{K}\left(L^{2}\left(\mathbb{R}^{m}\right)\right)$ é um subconjunto de $C_{o}\left(\mathbb{R}^{m}\right) \cap L^{2}\left(\mathbb{R}^{m}\right) \cap L^{1}\left(\mathbb{R}^{m}\right)$;

(ii) O núcleo $\tilde{K}$ é um elemento de $\mathcal{A}_{o}(D)$ representável na forma

$$
\tilde{K}(u, v)=\hat{K}(u,-v)=\sum_{1}^{\infty} \lambda_{n}(\mathcal{K}) \hat{\phi}_{n}(u) \overline{\hat{\phi}_{n}(v)}, \quad u, v \in \mathbb{R}^{m}
$$

com convergência $L^{2}\left(\mathbb{R}^{m} \times \mathbb{R}^{m}\right)$, absoluta e uniforme, sendo $\left\{\hat{\phi_{n}}\right\}$ uma base ortonormal de $L^{2}\left(\mathbb{R}^{m}\right)$ formada por autofunções associadas aos autovalores $\left\{\lambda_{n}(\mathcal{K})\right\}$ do operador $\tilde{\mathcal{K}}$. Além disso $\tilde{\mathcal{K}}\left(L^{2}\left(\mathbb{R}^{m}\right)\right) \subset C_{o}\left(\mathbb{R}^{m}\right) \cap L^{2}\left(\mathbb{R}^{m}\right)$;

(iii) Os operadores positivos $\tilde{\mathcal{K}}$ e $\mathcal{K}$ são nucleares, com

$$
\operatorname{tr}(|\mathcal{K}|)=\operatorname{tr}(\mathcal{K})=\operatorname{tr}(\tilde{\mathcal{K}})=\operatorname{tr}(|\tilde{\mathcal{K}}|)=\sum_{1}^{\infty} \lambda_{n}(\mathcal{K})=\int_{\mathbb{R}^{m}} K(x, x) d x=\int_{\mathbb{R}^{m}} \tilde{K}(u, u) d u .
$$

(iv) Se $K \in \operatorname{Lip}^{\alpha, s}\left(\mathbb{R}^{m}\right)$, então

$$
\lambda_{n}(\mathcal{K})=O\left(n^{-1-\gamma / m}\right), \quad \gamma:=\alpha \frac{\beta-m}{\beta-m+\alpha+s} .
$$

$(v)$ Se $K$ é diferenciável com $\partial K / \partial x$ uniformemente contínua na diagonal, então

$$
\lambda_{n}(\mathcal{K})=O\left(n^{-1-\gamma / m}\right), \quad \gamma:=\frac{\beta-m}{\beta+1} .
$$

(vi) Se $K$ é diferenciável e $\partial K / \partial x \in \operatorname{Lip}_{A}^{\alpha, m}\left(\mathbb{R}^{m}\right)$, onde $A$ é constante, então

$$
\lambda_{n}(\mathcal{K})=O\left(n^{-1-\gamma / m}\right), \quad \gamma=:(1+\alpha) \frac{\beta-m}{\beta+1+\alpha} .
$$




\section{Referências Bibliográficas}

[1] Akhiezer, N. I. - Lectures on integral transforms. Translated from Russian by H. H. McFaden. Translations of Mathematical Monographs, 70. American Mathematical Society, RI, 1988.

[2] Berg, C., Christensen; J. P. R. and Ressel, P. - Harmonic analysis on semigroups: Theory of positive definite and related functions. Springer-Verlag, 1984.

[3] Buescu, J. - Positive integral operators in unbounded domains. J. Math. Anal. Appl. 296 (2004), no. 1, 244-255.

[4] Buescu, J.; Paixão, A. C.; Garcia, F.; Lourtie, I. - Positive-definiteness, integral equations and Fourier transforms. J. Integral Equations Appl. 16 (2004), no. 1, 3352.

[5] Buescu, J.; Paixão A.C. - Eigenvalues of positive definite integral operators in unbounded intervals. Positivity. 10 (2006), 627-646.

[6] Buescu, J.; Paixão, A.C. - Eigenvalue distribution of positive definite kernels on unbounded domains. Integral Equations and Operator Theory. vol. 57 (2007), no. 1, $19-41$.

[7] Buescu, J.; Paixão, A.C. - Eigenvalue distribution of Mercer-like kernels. Math. Nachr. 280 (2007), no. 9-10, 984-995.

[8] Carmeli, C.; De Vito, E.; Toigo, A. - Reproducing kernel Hilbert spaces and Mercer theorem. Preprint, 2005.

[9] Chang, C.; Ha, C. - On eigenvalues of differentiable positive definite kernels. Integral Equations and Operator Theory 33 (1999), 1-7. 
[10] Chang, C.; Ha, C. - Eigenvalues of differentiable positive definite kernels. SIAM J. Math. Anal.. 17 (1986), 2, 415-419.

[11] Cochran, J. A.; Lukas, M. A. - Differentiable positive definite kernels and Lipschitz continuity. Math. Proc. Camb. Phil. Soc. 104 (1988), 361-369.

[12] Folland, G. B. - Real analysis: Modern techniques and their applications. John Wiley \& Sons, 1999.

[13] Folland, G. B. - Fourier analysis and its aplications. Wadsworth \& Brooks/Cole Advanced Books \& Software, 1992.

[14] Gohberg, I.; Gohberg, S.; Krupnik, N. - Traces and determinants of linear operators. Operator Theory: Advances and Applications, 116. Birkhäuser Verlag, Basel, 2000.

[15] Gohberg, M.; Krein, M. - Introduction to the theory of linear nonselfadjoint operators in Hilbert space. A. M. S., Providence, 1969.

[16] Groemer, H. - Geometric applications of Fourier series and spherical harmonics. Encyclopedia of Mathematics and its Applications, 61. Cambridge University Press, Cambridge, 1996.

[17] Horn, R. A.; Johnson C. R. - Matrix analysis. Cambridge University Press, 1991.

[18] Hubbert, S.; Müller, S.- Interpolation with circular basis functions. Numer. Algor.. 42 (2006), 75-90.

[19] Kadota, T. - Term by term differentiability of Mercer's expansion. Proc. Amer. math. Soc.. 18 (1967), 69-72.

[20] Kühn, T. - Eigenvalues of integral operators generated by Hölder continuous kernels on metric compacta. Nederl. Akad. Wetensch. Indag. Math. 49 (1987), no. 1, $51-61$.

[21] Kühn, T. - Eigenvalues of integral operators with smooth positive definite kernels. Arch. Math., Vol.49 (1987), 525-534.

[22] Kühn, T.; Cobos, F. - Eigenvalues of integral operators with positive definite kernels satisfying integrated Hölder conditions over metric compacta. J. Approx. Theory, 63 (1990), 39-55.

[23] Lima, E. L. - Espaços métricos. IMPA, 2003. 
[24] Lima, E. L. - Análise no espaço $R^{n}$. Editora Universidade de Brasília,1970.

[25] Lima, E. L. - Curso de análise Vol. 2. IMPA, 1981.

[26] Mercer, J. - Functions of positive and negative type and their connection with the theory of integral equations. Philos. Trans. R. Soc. Lond. Ser. A 209 (1909), 415-446.

[27] Munkres,J. R. - Topology, a first course. Prentice-Hall, 1975.

[28] Novitskiı̌, I. M. - Representation of kernels of integral operators by bilinear series. Siberiam Math. J. 25 (1984), no. 5, 774-778.

[29] Reade, J. - Eigenvalues of positive definite kernels. SIAM J. Math. Anal.. vol. 14 (1983),no. 1, 135-140.

[30] Reade, J. - Eigenvalues of positive definite kernels II. SIAM J. Math. Anal.. vol. 15 (1984),no. 1, 137-142.

[31] Reade, J. - On the sharpness of Weyl's estimate for eigenvalues of smooth kernels. SIAM J. Math. Anal.. vol. 16 (1985),no. 3, 137-142.

[32] Reade, J. - On the sharpness of Weyl's estimate for eigenvalues of smooth kernels II. SIAM J. Math. Anal.. vol. 19 (1988),no. 3, 137-142.

[33] Reed M.; Simon, B. - Methods of modern mathematical physics I: Functional analysis. Academic Press, 1972.

[34] Riesz; F.; Sz.-Nagy, B. - Functional analysis. Translated from the $2^{\text {nd }}$ French edition by Leo F. Boron. Frederick Ungar Publishing CO,1972.

[35] Rudin, W. - Principles of mathematical analysis. Third edition. International Series in Pure and Applied Mathematics. McGraw-Hill Book Co., New York-AucklandDüsseldorf, 1976.

[36] Schaback, R. - Native Hilbert spaces for radial basis functions I. New devolopments in approximation theory (Dortmund, 1998), 255-282, Internat. Ser. Numer. Math., 132, Birkhäuser, Basel, 1999.

[37] Schaback, R. - A unified theory of radial basis functions. Native Hilbert spaces for radial basis functions II. Numerical analysis in the 20th century, vol. I, Approximation theory. J. Comput. and Appl. Math. 121 (2000), no.1-2, 165-177. 
[38] Schoeberg, I. J. - Metric spaces and positive definite functions. Translation of the American Mathematical Society, Vol. 44, No. 3.(Nov.,1938), 522-536.

[39] Stewart, J. - Positive definite functions and generalizations, an historical survey. Rocky Mountain J. Math. 6 (1976), no. 3, 409-434.

[40] Sunder, V. S. - Functional analysis: spectral theory. Birkhäuser, 1998.

[41] Weidmann, J. - Linear operators in Hilbert spaces. Springer-Verlag, 1980.

[42] Wheeden, R. L.; Zygmund, A.- Measure and Integral: An introduction to real analysis. Marcel Dekker, 1977.

[43] Young, N. - An introduction to Hilbert space. Cambridge University Press, 1988. 


\section{Índice Remissivo}

$C_{o}(X, V), 29$

$K_{x}, K^{y}, 29$

$L^{2} P D(X), 25$

$\operatorname{Lip}^{\alpha, s}(X), 66$

$P D(X), 21$

$S^{m}$, esfera unitária de $\mathbb{R}^{m+1}, 39,78$

$T \geq 0$, operador positivo, 12

$T^{*}$, operador adjunto de $T, 11$

$X_{r}, D_{r}, 64$

$\mathcal{A}_{o}(X), 40$

$\mathcal{K}$, operador integral com núcleo $K, 16$

$\dot{K}, 23$

$\hat{f}$, transformada de Fourier de $f, 19,20$

$\lambda(T)$, autovalor do operador $T, 14$

$\langle\cdot, \cdot\rangle$, produto interno usual de $L^{2}(X), 7$

$\langle\cdot, \cdot\rangle_{\mathcal{H}}$, produto interno de $\mathcal{H}, 10$

$\mathbb{C}$, conjunto dos números complexos, 1

$\mathbb{N}$, conjunto dos números naturais, 2

$\mathbb{R}$, conjunto dos números reais, 2

$\mathcal{H}$, espaço de Hilbert separável, 10

$\mathcal{L}(\mathcal{X}, \mathcal{Y}), \mathcal{L}(X), 11$

$\operatorname{Im} T$, imagem de $T, 60$

$|T|, 13$

$\|\cdot\|_{\mathcal{X}}$, norma do espaço vetorial $\mathcal{X}, 10$

$\|\cdot\|_{t r}$, norma traço, 14

$|\cdot|$, norma euclideana de $\mathbb{R}^{m}, 3$

$\phi \otimes \psi, 17$
$\sqrt{T}, 13$

$\tilde{K}, 47$

$a_{n}=O\left(b_{n}\right), a_{n}=o\left(b_{n}\right), 6$

$f(x)=O\left(|x|^{\alpha}\right), f(x)=o\left(|x|^{\alpha}\right), 44$

$s_{n}(T)$, autovalores do operador $|T|, 15$

$\operatorname{tr}(T)$, traço do operador $T, 13,14$

Conjunto d-mensurável, 27

continuidade uniforme na diagonal, 71

Desigualdade

de Bessel, 10

de Cauchy-Schwarz, 10

de Hölder, 8

Identidade de Parseval, 19

Lema

da raiz quadrada, 13

de Riemann-Lebesgue, 19

matriz não-negativa definida, 1

medida estritamente positiva, 40

núcleo hermitiano, 17

operadores

auto-adjuntos, 11

compactos, 12

do tipo Hilbert-Schmidt, 15

integrais, 16 
normais, 11

nucleares, 13

positivos, 12

Teorema

da Convergência Dominada, 8

da Convergência Monótona, 8

da Diferenciação de Lebesgue, 9

da Mudança de Variáveis em Integrais, 9

de Dini, 2

de Hilbert-Schmidt, 14

de Mercer, 39

de Plancherel, 19

de Riesz-Fischer, 10

de Weierstrass, 2

transformada de Fourier, 19 